\title{
Character Theory of Finite Groups
}

\author{
Jun.-Prof. Dr. Caroline Lassueur \\ TU Kaiserslautern
}

Skript zur Vorlesung, SS 2020

(Vorlesung: 2SWS // Übung: 1SWS)

Version: 13. Juli 2020 
Foreword $\quad$ iii

Conventions $\quad v$

$\begin{array}{ll}\text { Chapter 1. Linear Representations of Finite Groups } & 7\end{array}$

1 Linear Representations . . . . . . . . . . . . . . . . . . . . . . 7

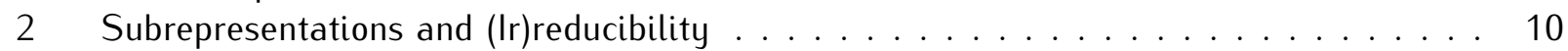

3 Maschke's Theorem . . . . . . . . . . . . . . . . . . . . . . . . . . . . . 12

Chapter 2. The Group Algebra and Its Modules 15

4 Modules over the Group Algebra . . . . . . . . . . . . . . . . . . . . . . 15

5 Schur's Lemma and Schur's Relations . . . . . . . . . . . . . . . . . . . . . . . . 18

$6 \quad \mathbb{C}$-Representations of Finite Abelian Groups . . . . . . . . . . . . . . . . . . 20

Chapter 3. Characters of Finite Groups 23

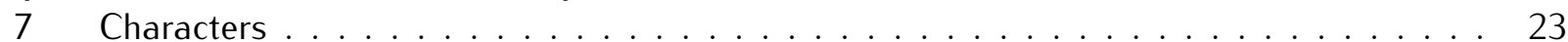

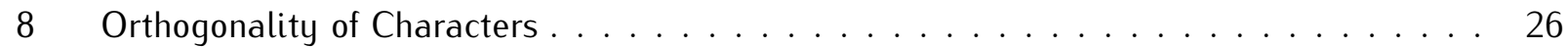

9 Consequences of the 1 st Orthogonality Relations . . . . . . . . . . . . . . . . 28

10 The Regular Character . . . . . . . . . . . . . . . . . . . . . . . . . 32

Chapter 4. The Character Table 34

11 The Character Table of a Finite Group . . . . . . . . . . . . . . . . . . . . 34

12 The 2nd Orthogonality Relations . . . . . . . . . . . . . . . . . . . 36

13 Tensor Products of Representations and Characters . . . . . . . . . . . . . . . . . 37

14 Normal Subgroups and Inflation . . . . . . . . . . . . . . . . . . . . 38

Chapter 5. Integrality and Theorems of Burnside's 44

15 Algebraic Integers and Character Values . . . . . . . . . . . . . . . . . . . . . 44

16 Central Characters . . . . . . . . . . . . . . . . . . . . . . . . . 45

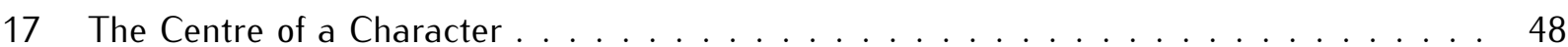

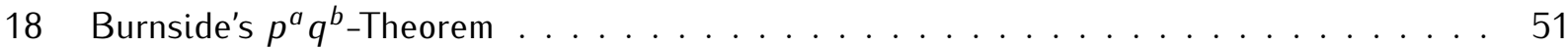


Chapter 6. Induction and Restriction of Characters 54

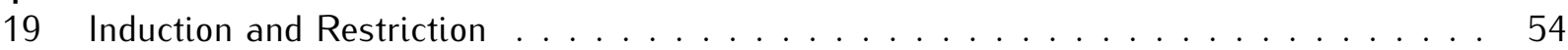

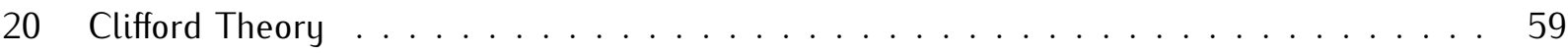

21 The Theorem of Gallagher. . . . . . . . . . . . . . . . . . 62

Appendix: Complements on Algebraic Structures

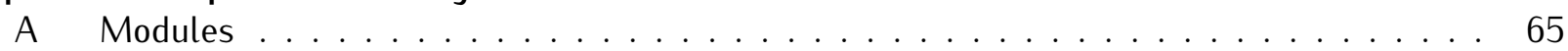

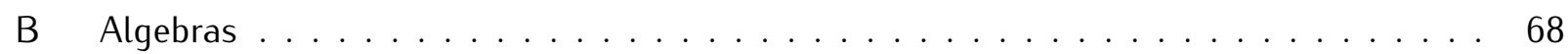

C Tensor Products of Vector Spaces . . . . . . . . . . . . . . . . . . . 69

D Integrality and Algebraic Integers . . . . . . . . . . . . . . . . . 71

$\begin{array}{ll}\text { Index of Notation } & 73\end{array}$ 
This text constitutes a faithful transcript of the lecture Character Theory of Finite Groups held at the TU Kaiserslautern during the Summer Semester 2020 (14 Weeks, 2SWS Lecture + 1SWS Exercises).

Together with the necessary theoretical foundations the main aims of this lecture are to:

- provide students with a modern approach to finite group theory;

- learn about the ordinary character theory of finite groups;

- learn about the applications of the latter theory to finite group theory, such as for example the proof of Burnside's $p^{a} q^{b}$-Theorem.

We assume as pre-requisites bachelor-level algebra courses dealing with linear algebra and elementary group theory, such as the standard lectures Grundlagen der Mathematik, Algebraische Strukturen, and Einführung in die Algebra.

The exercises mentioned in the text are important for the development of the lecture and the general understanding of the topics. Further exercises can be found in the fortnightly exercise sheets.

The content of the appendix is important for the lecture. Some of you may have encountered (or will encounter) this material in other algebra lectures. For this reason, no direct question on the appendix will be asked in the oral exam.

Books and lecture notes which were used to prepare these lecture notes are the following.

\section{Textbooks:}

[Dor71] L. Dornhoff. Group representation theory. Part A: Ordinary representation theory. Marcel Dekker, Inc., New York, 1971.

[Hup98] B. Huppert. Character theory of finite groups. Vol. 25. Walter de Gruyter \& Co., Berlin, 1998.

[Isa94] I. M. Isaacs. Character theory of finite groups. Dover Publications, Inc., New York, 1994.

[JL01] G. James and M. Liebeck. Representations and characters of groups. Second. Cambridge University Press, New York, 2001.

[LP10] K. Lux and H. Pahlings. Representations of groups. Vol. 124. Cambridge University Press, Cambridge, 2010. 
[NT89] H. Nagao and Y. Tsushima. Representations of finite groups. Academic Press, Inc., Boston, MA, 1989.

[Rot10] J. J. Rotman. Advanced modern algebra. 2nd ed. Providence, RI: American Mathematical Society (AMS), 2010.

[Ser77] J.-P. Serre. Linear representations of finite groups. Springer-Verlag, New York-Heidelberg, 1977.

[Ser78] J.-P. Serre. Représentations linéaires des groupes finis. revised. Hermann, Paris, 1978.

[Web16] P. Webb. A course in finite group representation theory. Vol. 161. Cambridge University Press, Cambridge, 2016.

\section{Lecture Notes:}

[Gec14] M. Geck. Algebra: Gruppen, Ringe, Körper (mit einer Einführung in die Darstellungstheorie endlicher Gruppen). Edition Delkhofen, 2014.

[Kül13] B. Külshammer. Darstellungstheorie. 2013. URL: http : / / www . minet . uni - jena . de / algebra/skripten/dt/dt-2010/dt.pdf.

[Mal16] G. Malle. Characters of finite groups. Lecture Notes SS 2016, TU Kaiserslautern. 2016.

[Thé05] J. Thévenaz. Représentations linéaires des groupes finis. Lecture Notes WS 2004/05, EPFL. 2005.

Acknowledgement: I am grateful to Gunter Malle who provided me with the Skript of his lecture "Charaktertheorie" held at the TU Kaiserslautern in the SS 2015, SS 2016, and SS 2018, which was used as a basis for the development of this lecture. I am also grateful to Birte Johannson and Bernhard Böhmler for reading and commenting a preliminary version of these notes. Further comments, corrections and suggestions are welcome.

Kaiserslautern, April 2020

Caroline Lassueur 
Unless otherwise stated, throughout these notes we make the following general assumptions:

- all groups considered are finite;

- all vector spaces considered are finite-dimensional;

- all rings considered are associative and unital (i.e. possess a neutral element for the multiplication, denoted 1);

- all modules considered are left modules. 
Chapter 1. Linear Representations of Finite Groups

Representation theory of finite groups is originally concerned with the ways of writing a finite group $G$ as a group of matrices, that is using group homomorphisms from $G$ to the general linear group $G_{n}(K)$ of invertible $n \times n$-matrices with coefficients in a field $K$ for some positive integer $n$.

Notation: throughout this chapter, unless otherwise specified, we let:

- $G$ denote a finite group (in multiplicative notation);

- $K$ denote a field of arbitrary characteristic; and

- $V$ denote a $K$-vector space such that $\operatorname{dim}_{K}(V)<\infty$.

In general, unless otherwise stated, all groups considered are assumed to be finite and all $K$-vector spaces considered are assumed to be finite-dimensional.

\section{Linear Representations}

\section{Definition 1.1 (K-representation, matrix representation, faithfullness)}

(a) A $K$-representation of $G$ (or a (linear) representation of $G$ (over $K)$ ) is a group homomorphism

$$
\rho: G \longrightarrow \operatorname{GL}(V),
$$

where $V$ is a $K$-vector space of dimension $n \in \mathbb{Z}_{>0}$. (Here $\mathrm{GL}(V):=\operatorname{Aut}_{K}(V)$ is the group of invertible $K$-endomorphisms of $V$.)

(b) A matrix representation of $G$ is a group homomorphism $R: G \longrightarrow G L_{n}(K)$, where $n \in \mathbb{Z}>0$.

In both cases the integer $n$ is called the degree of the representation. An injective (matrix) representation of $G$ is called faithful.

\section{Remark 1.2}

We see at once that both concepts of a representation and of a matrix representation are closely connected.

Recall that every choice of an ordered basis $B$ of $V$ yields a group isomorphism 


$$
\begin{aligned}
\alpha_{B}: G L(V) & \longrightarrow \mathrm{GL}_{n}(K) \\
\varphi & \mapsto(\varphi)_{B}
\end{aligned}
$$

where $(\varphi)_{B}$ denotes the matrix of $\varphi$ in the basis $B$. Therefore, a $K$-representation $\rho: G \longrightarrow \operatorname{GL}(V)$ together with the choice of an ordered basis $B$ of $V$ gives rise to a matrix representation of $G$ :

$$
R_{B}:=\alpha_{B} \circ \rho: G \stackrel{\rho}{\longrightarrow} \mathrm{GL}(V) \stackrel{\alpha_{B}}{\longrightarrow} \mathrm{GL}_{n}(K)
$$

Explicitly, $R_{B}$ sends an element $g \in G$ to the matrix $(\rho(g))_{B}$ of $\rho(g)$ in the basis $B$.

Another choice of a $K$-basis of $V$ yields another matrix representation!!

Conversely, any matrix representation $R: G \longrightarrow \mathrm{GL}_{n}(K)$ gives rise to a $K$-representation

$$
\begin{aligned}
\rho: \quad G & \longrightarrow \mathrm{GL}\left(K^{n}\right) \\
g & \mapsto \rho(g): K^{n} \longrightarrow K^{n}, v \mapsto R(g) v
\end{aligned}
$$

where we see $v$ as a column vector expressed in the standard basis of $K^{n}$ and $R(g) v$ is then the standard matrix multiplication. (Here we set $V:=K^{n}$.)

Throughout the lecture, we will favour the approach using representations rather than matrix representations in order to develop theoretical results. However, matrix representations are essential to carry out computations. Being able to pass back and forth from one approach to the other will be an essential feature.

Also note that Remark 1.2 allows us to transfer terminology/results from representations to matrix representations and conversely. Hence, from now on, in general we make new definitions for representations and use them for matrix representations as well.

\section{Example 1}

(a) If $G$ is an arbitrary finite group and $V:=K$, then

$$
\begin{aligned}
\rho: \quad G & \longrightarrow \mathrm{GL}(K) \cong K^{\times} \\
g & \mapsto \rho(g):=\mathrm{Id}_{K} \leftrightarrow 1_{K}
\end{aligned}
$$

is a $K$-representation of $G$, called the trivial representation of $G$.

Similarly $\rho: G \longrightarrow \mathrm{GL}(V), g \mapsto \mathrm{Id} V$ with $\operatorname{dim}_{K}(V)=: n>1$ is also a $K$-representation of $G$ and is called a trivial representation of $G$ of degree $n$.

(b) If $G$ is a subgroup of $G L(V)$, then the canonical inclusion

$$
\begin{aligned}
G & \hookrightarrow \mathrm{GL}(V) \\
g & \mapsto g
\end{aligned}
$$

is a faithful representation of $G$, called the tautological representation of $G$.

(c) Let $G:=S_{n}(n \geqslant 1)$ be the symmetric group on $n$ letters. Let $\left\{e_{1}, \ldots, e_{n}\right\}$ be the standard basis of $V:=K^{n}$. Then

$$
\begin{aligned}
\rho: S_{n} & \longrightarrow \mathrm{GL}\left(K^{n}\right) \\
\sigma & \mapsto \rho(\sigma): K^{n} \longrightarrow K^{n}, e_{i} \mapsto e_{\sigma(i)}
\end{aligned}
$$

is a $K$-representation, called the natural representation of $S_{n}$. 
(d) More generally, if $X$ is a finite $G$-set, i.e. a finite set endowed with a left action $: G \times X \longrightarrow X$, and $V$ is a $K$-vector space with basis $\left\{e_{x} \mid x \in X\right\}$, then

$$
\begin{aligned}
\rho_{X}: \quad G & \longrightarrow \mathrm{GL}(V) \\
g & \mapsto \rho_{X}(g): V \longrightarrow V, e_{X} \mapsto e_{g \cdot X}
\end{aligned}
$$

is a $K$-representation of $G$, called the permutation representation associated with $X$.

Notice that (c) is a special case of (d) with $G=S_{n}$ and $X=\{1,2, \ldots, n\}$.

If $X=G$ and the left action $: G \times X \longrightarrow X$ is just the multiplication in $G$, then

$$
\rho_{X}=: \rho_{\text {reg }}
$$

is called the regular representation of $G$.

We shall see later on in the lecture that $K$-representations are a special case of a certain algebraic structure (in the sense of the lecture Algebraische Strukturen). Thus, next, we define the notions that shall correspond to a homomorphism and an isomorphism of this algebraic structure.

\section{Definition 1.3 (Homomorphism of representations, equivalent representations)}

Let $\rho_{1}: G \longrightarrow \mathrm{GL}\left(V_{1}\right)$ and $\rho_{2}: G \longrightarrow \mathrm{GL}\left(V_{2}\right)$ be two $K$-representations of $G$, where $V_{1}, V_{2}$ are two non-zero $K$-vector spaces.

(a) A K-homomorphism $\alpha: V_{1} \longrightarrow V_{2}$ such that $\rho_{2}(g) \circ \alpha=\alpha \circ \rho_{1}(g)$ for each $g \in G$ is called a homomorphism of representations (or a $G$-homomorphism) between $\rho_{1}$ and $\rho_{2}$.

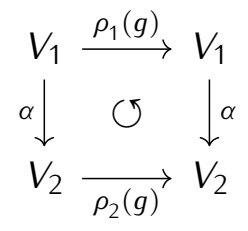

(b) If, moreover, $\alpha$ is a $K$-isomorphism, then it is called an isomorphism of representations (or a $G$-isomorphism), and the $K$-representations $\rho_{1}$ and $\rho_{2}$ are called equivalent (or isomorphic). In this case we write $\rho_{1} \sim \rho_{2}$.

(c) Two matrix representations $R_{1}, R_{2}: G \longrightarrow \mathrm{GL}_{n}(K)$ are called equivalent iff $\exists T \in \mathrm{GL}_{n}(K)$ such that

$$
R_{2}(g)=T R_{1}(g) T^{-1} \quad \forall g \in G .
$$

In this case we write $R_{1} \sim R_{2}$.

\section{Remark 1.4}

(a) Equivalent representations have the same degree.

(b) Clearly $\sim$ is an equivalence relation.

(c) In consequence, it essentially suffices to study representations up to equivalence (as it essentially suffices to study groups up to isomorphism). 


\section{Remark 1.5}

If $\rho: G \longrightarrow \mathrm{GL}(V)$ is a $K$-representation of $G$ and $E:=\left(e_{1}, \ldots, e_{n}\right), F:=\left(f_{1}, \ldots, f_{n}\right)$ are two ordered bases of $V$, then by Remark 1.2, we have two matrix representations:

$$
\begin{aligned}
& R_{E}: G \longrightarrow \mathrm{GL}_{n}(K) \quad \text { and } \quad R_{F}: G \longrightarrow \mathrm{GL}_{n}(K) \\
& g \mapsto(\rho(g))_{E} \quad \text { and } \quad g \quad \mapsto \quad(\rho(g))_{F}
\end{aligned}
$$

These matrix representations are equivalent since $R_{F}(g)=T R_{E}(g) T^{-1} \forall g \in G$, where $T$ is the change-of-basis matrix.

\section{Subrepresentations and (Ir)reducibility}

Subrepresentations allow us to introduce one of the main notions that will enable us to break representations in elementary pieces in order to simplify their study: the notion of (ir)reducibility.

\section{Definition 2.1 (G-invariant subspace, irreducibility)}

Let $\rho: G \longrightarrow \mathrm{GL}(V)$ be a $K$-representation of $G$.

(a) A $K$-subspace $W \subseteq V$ is called $G$-invariant if

$$
\rho(g)(W) \subseteq W \quad \forall g \in G .
$$

(In fact in this case the reverse inclusion holds as well, since for each $w \in W$ we can write $w=\rho\left(g g^{-1}\right)(w)=\rho(g)\left(\rho\left(g^{-1}\right)(w)\right) \in \rho(g)(W)$, hence $\left.\rho(g)(W)=W.\right)$

(b) The representation $\rho$ is called irreducible if it admits exactly two $G$-invariant subspaces: 0 and $V$ itself. Else it is called reducible (i.e. if it admits a non-trivial proper $G$-invariant subspace $\{0\} \subsetneq W \subsetneq V$ ).

Notice that $V$ itself and the zero subspace 0 are always $G$-invariant subspaces.

\section{Definition 2.2 (Subrepresentation)}

If $\rho: G \longrightarrow \mathrm{GL}(V)$ is a $K$-representation and $0 \neq W \subseteq V$ is a $G$-invariant subspace, then

$$
\begin{aligned}
\rho_{W}: G & \longrightarrow \operatorname{GL}(W) \\
g & \mapsto \rho_{W}(g):=\left.\rho(g)\right|_{W}: W \longrightarrow W
\end{aligned}
$$

is called a subrepresentation of $\rho$. (This is clearly again a representation of $G$.)

With this definition, it is clear that a representation $\rho: G \longrightarrow \mathrm{GL}(V)$ is irreducible if and only if $\rho$ does not possess any proper subrepresentation.

\section{Remark 2.3}

Let $\rho: G \longrightarrow \mathrm{GL}(V)$ be a $K$-representation and $0 \neq W \subseteq V$ be a $G$-invariant subspace. Now choose an ordered basis $B^{\prime}$ of $W$ and complete it to an ordered basis $B$ of $V$. Then for each $g \in G$ 
the corresponding matrix representation is of the form

$$
(\rho(g))_{B}=\left[\begin{array}{c|c}
\left(\rho_{W}(g)\right)_{B^{\prime}} & * \\
\hline 0 & \multirow{B}{*}{\begin{array}{c}
* \\
B^{\prime}
\end{array}}
\end{array}\right] .
$$

\section{Example 2}

(a) Any $K$-representation of degree 1 is irreducible.

(b) Let $\rho: S_{n} \longrightarrow \mathrm{GL}\left(K^{n}\right)$ be the natural representation of $S_{n}(n \geqslant 1)$ and let $B:=\left(e_{1}, \ldots, e_{n}\right)$ be the standard basis of $V=K^{n}$. Then for each $g \in G$ we have

$$
\rho(g)\left(\sum_{i=1}^{n} e_{i}\right)=\sum_{i=1}^{n} \rho(g)\left(e_{i}\right)=\sum_{i=1}^{n} e_{i}
$$

where the last equality holds because $\rho(g):\left\{e_{1}, \ldots, e_{n}\right\} \longrightarrow\left\{e_{1}, \ldots, e_{n}\right\}, e_{i} \mapsto e_{g(i)}$ is a bijection. Thus

$$
W:=\left\langle\sum_{i=1}^{n} e_{i}\right\rangle_{K}
$$

is an $S_{n}$-invariant subspace of $K^{n}$ of dimension 1. It follows that $\rho$ is reducible if $n>1$.

(c) More generally, the trivial representation of a finite group $G$ is a subrepresentation of any permutation representation of $G$. [Exercise 2(a), Sheet 1]

(d) The symmetric group $S_{3}=\left\langle\left(\begin{array}{ll}1 & 2\end{array}\right),\left(\begin{array}{lll}1 & 2 & 3\end{array}\right)\right\rangle$ admits the following three non-equivalent irreducible matrix representations over $\mathbb{C}$ :

$$
\rho_{1}: S_{3} \longrightarrow \mathbb{C}^{\times}, \sigma \mapsto 1
$$

i.e. the trivial representation,

$$
\rho_{2}: S_{3} \longrightarrow \mathbb{C}^{\times}, \sigma \mapsto \operatorname{sign}(\sigma)
$$

where $\operatorname{sign}(\sigma)$ denotes the sign of the permutation $\sigma$, and

$$
\begin{array}{rlll}
\rho_{3}: & S_{3} & \longrightarrow & \mathrm{GL}_{2}(\mathbb{C}) \\
\left(\begin{array}{lll}
1 & 2
\end{array}\right) & \mapsto & \left(\begin{array}{ll}
0 & 1 \\
1 & 0
\end{array}\right) \\
\left(\begin{array}{lll}
1 & 2 & 3
\end{array}\right) & \mapsto & \left(\begin{array}{ll}
0 & -1 \\
1 & -1
\end{array}\right) .
\end{array}
$$

See [Exercise 1(a), Sheet 1].

We will prove later in the lecture that these are all the irreducible $\mathbb{C}$-representations of $S_{3}$ up to equivalence. 


\section{Properties 2.4}

Let $\rho_{1}: G \longrightarrow \mathrm{GL}\left(V_{1}\right)$ and $\rho_{2}: G \longrightarrow \mathrm{GL}\left(V_{2}\right)$ be two $K$-representations of $G$ and let $\alpha: V_{1} \longrightarrow V_{2}$ be a $G$-homomorphism.

(a) If $W \subseteq V_{1}$ is a $G$-invariant subspace of $V_{1}$, then $\alpha(W) \subseteq V_{2}$ is $G$-invariant.

(b) If $W \subseteq V_{2}$ is a $G$-invariant subspace of $V_{2}$, then $\alpha^{-1}(W) \subseteq V_{1}$ is $G$-invariant.

(c) In particular, $\operatorname{ker}(\alpha)$ and $\operatorname{Im}(\alpha)$ are $G$-invariant subspaces of $V_{1}$ and $V_{2}$ respectively.

Proof: [Exercise 3, Sheet 1].

\section{Maschke's Theorem}

We now come to our first major result in the representation theory of finite groups, namely Maschke's Theorem, which provides us with a criterion for representations to decompose into direct sums of irreducible subrepresentations.

\section{Definition 3.1 (Direct sum of subrepresentations)}

Let $\rho: G \longrightarrow \mathrm{GL}(V)$ be a $K$-representation. If $0 \neq W_{1}, W_{2} \subseteq V$ are two $G$-invariant subspaces such that $V=W_{1} \oplus W_{2}$, then we say that $\rho$ is the direct sum of the subrepresentations $\rho_{W_{1}}$ and $\rho_{W_{2}}$ and we write $\rho=\rho_{W_{1}} \oplus \rho_{W_{2}}$.

\section{Remark 3.2}

With the notation of Definition 3.1, if we choose an ordered basis $B_{i}$ of $W_{i}(i=1,2)$ and consider the ordered $K$-basis $B:=B_{1} \sqcup B_{2}$ of $V$, then the corresponding matrix representation is of the form

$$
(\rho(g))_{B}=\left[\begin{array}{c|c}
\left(\rho_{W_{1}}(g)\right)_{B_{1}} & 0 \\
\hline 0 & \left(\rho_{W_{2}}(g)\right)_{B_{2}}
\end{array}\right] \quad \forall g \in G .
$$

The following exercise shows that it is not always possible to decompose representations into direct sums of irreducible subrepresentations.

\section{Exercise 3.3 (Exercise 4, Sheet 1)}

Let $p$ be an odd prime number, let $G:=C_{p}=\left\langle g \mid g^{p}=1\right\rangle$, let $K:=\mathbb{F}_{p}$, and let $V:=\mathbb{F}_{p}^{2}$ with its canonical basis $B=\left(e_{1}, e_{2}\right)$. Consider the matrix representation

$$
\begin{aligned}
R: \quad G & \longrightarrow G L_{2}(K) \\
g^{b} & \mapsto\left(\begin{array}{ll}
1 & b \\
0 & 1
\end{array}\right) .
\end{aligned}
$$

(a) Prove that $K e_{1}$ is $G$-invariant and deduce that $R$ is reducible. 
(b) Prove that there is no direct sum decomposition of $V$ into irreducible $G$-invariant subspaces.

\section{Theorem 3.4 (MASCHKE)}

Let $G$ be a finite group and let $\rho: G \longrightarrow \mathrm{GL}(V)$ be a $K$-representation of $G$. If $\operatorname{char}(K) \nmid|G|$, then every $G$-invariant subspace $W$ of $V$ admits a $G$-invariant complement in $V$, i.e. a $G$-invariant subspace $U \subseteq V$ such that $V=W \oplus U$.

Proof: To begin with, choose an arbitrary complement $U_{0}$ to $W$ in $V$, i.e. $V=W \oplus U_{0}$ as $K$-vector spaces.

(Note that, however, $U_{0}$ is possibly not $G$-invariant!) Next, consider the projection onto $W$ along $U_{0}$, that is the $K$-linear map

$$
\pi: V=W \oplus U_{0} \longrightarrow W
$$

which maps an element $v=w+u$ with $w \in W, u \in U_{0}$ to $w$, and define a new $K$-linear map

$$
\begin{aligned}
\tilde{\pi}: \quad V & \longrightarrow V \\
v & \mapsto \frac{1}{|G|} \sum_{g \in G} \rho(g) \pi \rho\left(g^{-1}\right)(v) .
\end{aligned}
$$

Notice that it is allowed to divide by $|G|$ because the hypothesis that $\operatorname{char}(K) \nmid|G|$ implies that $|G| \cdot 1_{K}$ is invertible in the field $K$.

We prove the following assertions:

(1) $\operatorname{Im} \tilde{\pi} \subseteq W$ : indeed, if $v \in V$, then

$$
\tilde{\pi}(v)=\frac{1}{|G|} \sum_{g \in G} \underbrace{\rho(g) \underbrace{\pi \rho\left(g^{-1}\right)(v)}_{\in W}}_{\in W \text { (G-invariance) }} \in W .
$$

(2) $\left.\tilde{\pi}\right|_{W}=\operatorname{Id}{ }_{W}$ : indeed, if $w \in W$, then

$$
\tilde{\pi}(w)=\frac{1}{|G|} \sum_{g \in G} \rho(g) \pi \underbrace{\pi \underbrace{\rho\left(g^{-1}\right)(w)}_{\begin{array}{c}
\in W \\
\text { (by }- \text { invariance) }
\end{array}}}_{\begin{array}{c}
=\rho\left(g^{-1}\right)(w) \\
\text { (by def. of } \pi \text { ) }
\end{array}}=\frac{1}{|G|} \sum_{g \in G} \underbrace{\rho(g) \rho\left(g^{-1}\right)}_{\begin{array}{c}
=\rho\left(g g^{-1}\right) \\
=\rho(1 G) \\
=d_{V}
\end{array}}(w)=\frac{1}{|G|} \sum_{g \in G} w=w .
$$

Thus $(1)+(2)$ imply that $\tilde{\pi}$ is a projection onto $W$ so that as a $K$-vector space

$$
V=W \oplus \operatorname{ker}(\tilde{\pi}) \text {. }
$$

(3) ker $\widetilde{\pi}$ is $G$-invariant: indeed, for each $h \in G$ we have

$$
\begin{aligned}
\rho(h) \circ \tilde{\pi} & =\frac{1}{|G|} \sum_{g \in G} \underbrace{\rho(h) \rho(g)}_{=\rho(h g)} \pi \rho\left(g^{-1}\right) \\
& =\frac{1}{|G|} \sum_{g \in G} \rho(h g) \pi \rho\left((h g)^{-1} h\right) \\
& :=h g \\
& =\frac{1}{|G|} \sum_{s \in G} \rho(s) \pi \rho\left(s^{-1} h\right) \\
& =\left(\frac{1}{|G|} \sum_{s \in G} \rho(s) \pi \rho\left(s^{-1}\right)\right) \rho(h)=\tilde{\pi} \circ \rho(h) .
\end{aligned}
$$

Hence $\tilde{\pi}$ is a $G$-homomorphism and it follows from Property 2.4(c) that its kernel is $G$-invariant. Therefore we may set $U:=\operatorname{ker}(\tilde{\pi})$ and the claim follows. 


\section{Definition 3.5 (Completely reducible/semisimple representation / constituent)}

A $K$-representation which can be decomposed into a direct sum of irreducible subrepresentations is called completely reducible or semisimple. In this case, an irreducible subrepresentation occuring in such a decomposition is called a constituent of the representation.

\section{Corollary 3.6}

If $G$ is a finite group and $K$ is a field such that $\operatorname{char}(K) \nmid|G|$, then every $K$-representation of $G$ is completely reducible.

Proof: Let $\rho: G \longrightarrow \mathrm{GL}(V)$ be a $K$-representation of $G$.

- Case 1: $\rho$ is irreducible $\Rightarrow$ nothing to do $\checkmark$.

- Case 2: $\rho$ is reducible. Thus $\operatorname{dim}_{K}(V) \geqslant 2$ and there exists an irreducible $G$-invariant subspace $0 \neq V_{1} \subseteq V$. Now, by Maschke's Theorem, there exists a $G$-invariant complement $U \subseteq V$, i.e. such that $V=V_{1} \oplus U$. As $\operatorname{dim}_{K}\left(V_{1}\right) \geqslant 1$, we have $\operatorname{dim}_{K}(U)<\operatorname{dim}_{K}(V)$. Therefore, an induction argument yields the existence of a decomposition

$$
V=V_{1} \oplus V_{2} \oplus \cdots \oplus V_{r} \quad(r \geqslant 2)
$$

of $V$, where $V_{1}, \ldots, V_{r}$ are irreducible $G$-invariant subspaces.

\section{Remark 3.7}

(a) The hypothesis of Maschke's Theorem requiring that $\operatorname{char}(K) \nmid|G|$ is always verified if $K$ is a field of characteristic zero. E.g. if $K=\mathbb{C}, \mathbb{R}, \mathbb{Q}, \ldots$

(b) The converse of Maschke's Theorem holds as well. It will be proved in the M.Sc. lecture Representation Theory.

(c) In the literature, a representation is called an ordinary representation if $K$ is a field of characteristic zero (or more generally of characteristic not dividing $|G|$ ), and it is called a modular representation if $\operatorname{char}(K)|| G \mid$.

In this lecture we are going to reduce our attention to ordinary representation theory and, most of the time, even assume that $K$ is the field $\mathbb{C}$ of complex numbers.

\section{Exercise 3.8 (Alternative proof of Maschke's Theorem over the field $\mathbb{C}$. Exercise 5, Sheet 2.)}

Assume $K=\mathbb{C}$ and let $\rho: G \longrightarrow \mathrm{GL}(V)$ be a $\mathbb{C}$-representation of $G$.

(a) Prove that there exists a $G$-invariant scalar product $\langle\rangle:, V \times V \longrightarrow \mathbb{C}$, i.e. such that

$$
\langle g . u, g . v\rangle=\langle u, v\rangle \quad \forall g \in G, \forall u, v \in V .
$$

[Hint: consider an arbitrary scalar product on $V$, say $():, V \times V \longrightarrow \mathbb{C}$, which is not necessarily $G$-invariant. Use a sum on the elements of $G$, weighted by the group order $|G|$, in order to produce a new $G$-invariant scalar product on $V$.]

(b) Deduce that every $G$-invariant subspace $W$ of $V$ admits a $G$-invariant complement.

[Hint: consider the orthogonal complement of $W$.] 
We now introduce the concept of a $K G$-module, and show that this more modern approach is equivalent to the concept of a $K$-representation of a given finite group $G$. Some of the material in the remainder of these notes will be presented in terms of $K G$-modules. As we will soon see with our second fundamental result - Schur's Lemma - there are several advantages to this approach to representation theory.

Notation: throughout this chapter, unless otherwise specified, we let:

- $G$ denote a finite group;

- $K$ denote a field of arbitrary characteristic; and

- $V$ denote a $K$-vector space such that $\operatorname{dim}_{K}(V)<\infty$.

In general, unless otherwise stated, all groups considered are assumed to be finite and all $K$-vector spaces / modules over the group algebra considered are assumed to be finite-dimensional.

\section{Modules over the Group Algebra}

\section{Lemma-Definition 4.1 (Group algebra)}

The group ring $K G$ is the ring whose elements are the $K$-linear combinations $\sum_{g \in G} \lambda_{g} g$ with $\lambda_{g} \in K$, and addition and multiplication are given by

$$
\sum_{g \in G} \lambda_{g} g+\sum_{g \in G} \mu_{g} g=\sum_{g \in G}\left(\lambda_{g}+\mu_{g}\right) g \quad \text { and } \quad\left(\sum_{g \in G} \lambda_{g} g\right) \cdot\left(\sum_{h \in G} \mu_{h} h\right)=\sum_{g, h \in G}\left(\lambda_{g} \mu_{h}\right) g h
$$

respectively. In fact $K G$ is a $K$-vector space with basis $G$, hence a $K$-algebra. Thus we usually call $K G$ the group algebra of $G$ over $K$ rather than simply group ring.

Note: In Definition 4.1, the field $K$ can be replaced with a commutative ring $R$. E.g. if $R=\mathbb{Z}$, then $\mathbb{Z} G$ is called the integral group ring of $G$.

Proof: By definition $K G$ is a $K$-vector space with basis $G$, and the multiplication in $G$ is extended by $K$-bilinearity to the given multiplication : $K G \times K G \longrightarrow K G$. It is then straightforward to check that $K G$ bears both the structures of a ring and of a $K$-vector space. Finally, axiom (A3) of $K$-algebras (see Appendix B) follows directly from the definition of the multiplication and the commutativity of $K$. 


\section{Remark 4.2}

Clearly $1_{K G}=1_{G}, \operatorname{dim}_{K}(K G)=|G|$, and $K G$ is commutative if and only if $G$ is an abelian group.

\section{Proposition 4.3}

(a) Any $K$-representation $\rho: G \longrightarrow \mathrm{GL}(V)$ of $G$ gives rise to a $K G$-module structure on $V$, where the external composition law is defined by the map

$$
\begin{array}{ccc}
\quad: \quad \begin{array}{c}
K G \times V \\
\left(\sum_{g \in G} \lambda_{g} g, v\right)
\end{array} & \mapsto \quad & \left(\sum_{g \in G} \lambda_{g} g\right) \cdot v:=\sum_{g \in G} \lambda_{g} \rho(g)(v) .
\end{array}
$$

(b) Conversely, every $K G$-module $(V,+, \cdot)$ defines a $K$-representation

$$
\begin{aligned}
\rho_{V}: \quad G & \longrightarrow \mathrm{GL}(V) \\
g & \mapsto \rho_{V}(g): V \longrightarrow V, v \mapsto \rho_{V}(g)(v):=g \cdot v
\end{aligned}
$$

of the group $G$.

Proof: (a) Since $V$ is a $K$-vectore space it is equipped with an internal addition + such that $(V,+)$ is an abelian group. It is then straightforward to check that the given external composition law defined above verifies the $K G$-module axioms.

(b) A $K G$-module is in particular a $K$-vector space for the scalar multiplication defined for all $\lambda \in K$ and all $v \in V$ by

$$
\lambda v:=(\underbrace{\lambda 1_{G}}_{\in K G}) \cdot v .
$$

Moreover, it follows from the $K G$-module axioms that $\rho_{V}(g) \in \mathrm{GL}(V)$ and also that

$$
\rho_{V}\left(g_{1} g_{2}\right)=\rho_{V}\left(g_{1}\right) \circ \rho_{V}\left(g_{2}\right)
$$

for all $g_{1}, g_{2} \in G$, hence $\rho_{V}$ is a group homomorphism.

See [Exercise 7, Sheet 2] for the details (Hint: use the remark below!).

\section{Remark 4.4}

In fact in Proposition 4.3(a) checking the $K G$-module axioms is equivalent to checking that for all $g, h \in G, \lambda \in K$ and $u, v \in V$ :

(1) $(g h) \cdot v=g \cdot(h \cdot v)$;

(2) $1_{G} \cdot v=v$;

(4) $g \cdot(u+v)=g \cdot u+g \cdot v$;

(3) $g \cdot(\lambda v)=\lambda(g \cdot v)=(\lambda g) \cdot v$,

or in other words, that the binary operation

$$
\begin{aligned}
: G \times V & \longrightarrow V \\
(g, v) & \mapsto g \cdot v:=\rho(g)(v)
\end{aligned}
$$

is a $K$-linear action of the group $G$ on $V$. Indeed, the external multiplication of $K G$ on $V$ is just the extension by $K$-linearity of the latter map. For this reason, sometimes, $K G$-modules are also called G-vector spaces. See [Exercise 6, Sheet 2] for the details. 


\section{Lemma 4.5}

Two representations $\rho_{1}: G \longrightarrow \mathrm{GL}\left(V_{1}\right)$ and $\rho_{2}: G \longrightarrow \mathrm{GL}\left(V_{2}\right)$ are equivalent if and only if $V_{1} \cong V_{2}$ as $K G$-modules.

Proof: If $\rho_{1} \sim \rho_{2}$ and $\alpha: V_{1} \longrightarrow V_{2}$ is a $K$-isomorphism such that $\rho_{2}(g)=\alpha \circ \rho_{1}(g) \circ \alpha^{-1}$ for each $g \in G$, then by Proposition 4.3(a) for every $v \in V_{1}$ and every $g \in G$ we have

$$
g \cdot \alpha(v)=\rho_{2}(g)(\alpha(v))=\alpha\left(\rho_{1}(g)(v)\right)=\alpha(g \cdot v) .
$$

Hence $\alpha$ is a $K G$-isomorphism.

Conversely, if $\alpha: V_{1} \longrightarrow V_{2}$ is a $K G$-isomorphism, then certainly it is a $K$-homomorphism and for each $g \in G$ and by Proposition 4.3(b) for each $v \in V_{2}$ we have

$$
\alpha \circ \rho_{1}(g) \circ \alpha^{-1}(v)=\alpha\left(\rho_{1}(g)\left(\alpha^{-1}(v)\right)=\alpha\left(g \cdot \alpha^{-1}(v)\right)=g \cdot \alpha\left(\alpha^{-1}(v)\right)=g \cdot v=\rho_{2}(g)(v),\right.
$$

hence $\rho_{2}(g)=\alpha \circ \rho_{1}(g) \circ \alpha^{-1}$ for each $g \in G$.

\section{Remark 4.6 (Dictionary)}

More generally, through Proposition 4.3, we may transport terminology and properties from $K G$ modules to representations and conversely.

This lets us build the following dictionary:

REPRESENTATIONS

K-representation of $G$

degree

homomorphism of representations

subrepresentation / G-invariant subspace

direct sum of representations $\rho_{V_{1}} \oplus \rho_{V_{2}}$

irreducible representation

the trivial representation

the regular representation of $G$

Corollary 3.6 to Maschke's Theorem:

If $\operatorname{char}(K) \nmid|G|$, then every $K$-represen-

tation of $G$ is completely reducible.
Modules

\section{$\longleftrightarrow \quad K G$-module}

$\longleftrightarrow$ K-dimension

$\longleftrightarrow$ homomorphism of $K G$-modules

$\longleftrightarrow K G$-submodule

$\longleftrightarrow$ direct sum of $K G$-modules $V_{1} \oplus V_{2}$

$\longleftrightarrow$ simple (= irreducible) $K G$-module

$\longleftrightarrow$ the trivial $K G$-module $K$

$\longleftrightarrow$ the regular $K G$-module $K G$

$\longleftrightarrow$ Corollary 3.6 to Maschke's Theorem:

If $\operatorname{char}(K) \nmid|G|$, then every $K G$-module is semisimple.

Virtually, any result, we have seen in Chapter 1, can be reinterpreted using this translation table. E.g. Property 2.4(c) tells us that the image and the kernel of homomorphisms of $K G$-modules are $K G$-submodules, ...

In this lecture, we introduce the equivalence between representations and modules for the sake of completeness. In the sequel we keep on stating results in terms of representations as much as possible. However, we will use modules when we find them more fruitful. In contrast, the M.Sc. Lecture Representation Theory will consistently use the module approach to representation theory. 


\section{Schur's Lemma and Schur's Relations}

Schur's Lemma is a basic result concerning simple modules, or in other words irreducible representations. Though elementary to state and prove, it is fundamental to representation theory of finite groups.

\section{Theorem 5.1 (SCHUR'S LEMMA)}

(a) Let $V, W$ be simple $K G$-modules. Then the following assertions hold.

(i) Any homomorphism of $K G$-modules $\varphi: V \longrightarrow V$ is either zero or invertible. In other words $\operatorname{End}_{K G}(V)$ is a skew-field.

(ii) If $V \not W$, then $\operatorname{Hom}_{K G}(V, W)=0$.

(b) If $K$ is an algebraically closed field and $V$ is a simple $K G$-module, then

$$
\operatorname{End}_{K G}(V)=\left\{\lambda \operatorname{ld}_{V} \mid \lambda \in K\right\} \cong K
$$

Notice that here we state Schur's Lemma in terms of modules, rather than in terms of representations, because part (a) holds in greater generality for arbitrary unital associative rings and part (b) holds for finite-dimensional algebras over an algebraically closed field.

\section{Proof:}

(a) First, we claim that every $\varphi \in \operatorname{Hom}_{K G}(V, W) \backslash\{0\}$ admits an inverse in $\operatorname{Hom}_{K G}(W, V)$. Indeed, $\varphi \neq 0 \Longrightarrow \operatorname{ker} \varphi \subsetneq V$ is a proper $K G$-submodule of $V$ and $\{0\} \neq \operatorname{Im} \varphi$ is a non-zero $K G$-submodule of $W$. But then, on the one hand, $\operatorname{ker} \varphi=\{0\}$, because $V$ is simple, hence $\varphi$ is injective, and on the other hand, $\operatorname{Im} \varphi=W$ because $W$ is simple. It follows that $\varphi$ is also surjective, hence bijective. Therefore, by Properties A.7, $\varphi$ is invertible with inverse $\varphi^{-1} \in \operatorname{Hom}_{K G}(W, V)$.

Now, (ii) is straightforward from the above. For (i), first recall that $\operatorname{End}_{K G}(V)$ is a ring (see Notation A.8), which is obviously non-zero as $\operatorname{End}_{K G}(V) \ni \mathrm{Id}_{V}$ and $\mathrm{Id}_{V} \neq 0$ because $V \neq 0$ since it is simple. Thus, as any $\varphi \in \operatorname{End}_{K G}(V) \backslash\{0\}$ is invertible, $\operatorname{End}_{K G}(V)$ is a skew-field.

(b) Let $\varphi \in \operatorname{End}_{K G}(V)$. Since $K=\bar{K}, \varphi$ has an eigenvalue $\lambda \in K$. Let $v \in V \backslash\{0\}$ be an eigenvector of $\varphi$ for $\lambda$. Then $\left(\varphi-\lambda I_{V}\right)(v)=0$. Therefore, $\varphi-\lambda I_{V}$ is not invertible and

$$
\varphi-\lambda \operatorname{Id}_{V} \in \operatorname{End}_{K G}(V) \stackrel{(a)}{\Longrightarrow} \varphi-\lambda \operatorname{Id}_{V}=0 \quad \Longrightarrow \quad \varphi=\lambda \operatorname{Id}_{V} .
$$

Hence $\operatorname{End}_{K G}(V) \subseteq\left\{\lambda I_{V} \mid \lambda \in K\right\}$, but the reverse inclusion also obviously holds, so that

$$
\operatorname{End}_{K G}(V)=\left\{\lambda \operatorname{ld}_{V}\right\} \cong K
$$

\section{Exercise 5.2 (Exercise 8, Sheet 2)}

Prove that in terms of matrix representations the following statement holds:

Lemma 5.3 (Schur's Lemma for matrix representations)

Let $R: G \longrightarrow \mathrm{GL}_{n}(K)$ and $R^{\prime}: G \longrightarrow \mathrm{GL}_{n^{\prime}}(K)$ be two irreducible matrix representations. If there exists $A \in M_{n \times n^{\prime}}(K) \backslash\{0\}$ such that $A R^{\prime}(g)=R(g) A$ for every $g \in G$, then $n=n^{\prime}$ and $A$ is invertible (in particular $R \sim R^{\prime}$ ). 
The next lemma is a general principle, which we have already used in the proof of Maschke's Theorem, and which allows us to transform $K$-linear maps into $K G$-linear maps.

\section{Lemma 5.4}

Assume $\operatorname{char}(K) \nmid|G|$. Let $V, W$ be two $K G$-modules and let $\rho_{V}: G \longrightarrow \mathrm{GL}(V), \rho_{W}: G \longrightarrow \mathrm{GL}(W)$ be the associated $K$-representations. If $\psi: V \longrightarrow W$ is $K$-linear, then the map

$$
\widetilde{\psi}:=\frac{1}{|G|} \sum_{g \in G} \rho_{W}(g) \circ \psi \circ \rho_{V}\left(g^{-1}\right)
$$

from $V$ to $W$ is $K G$-linear.

Proof: Same argument as in (3) of the proof of Maschke's Theorem: replace $\pi$ by $\psi$ and apply the fact that a $G$-homomorphism between representations corresponds to a $K G$-hmomorphism between the corresponding $K G$-modules.

\section{Proposition 5.5}

Assume $\operatorname{char}(K) \nmid|G|$. Let $\rho_{V}: G \longrightarrow \mathrm{GL}(V)$ and $\rho_{W}: G \longrightarrow \mathrm{GL}(W)$ be two irreducible $K$ representations.

(a) If $\rho_{V} \nsim \rho_{W}$ and $\psi: V \longrightarrow W$ is a $K$-linear map, then

$$
\widetilde{\psi}=\frac{1}{|G|} \sum_{g \in G} \rho_{W}(g) \circ \psi \circ \rho_{V}\left(g^{-1}\right)=0 .
$$

(b) Assume moreover that $K=\bar{K}$ and $\operatorname{char}(K) \nmid n:=\operatorname{dim}_{K} V$. If $\psi: V \longrightarrow V$ is a $K$-linear map, then

$$
\widetilde{\psi}:=\frac{1}{|G|} \sum_{g \in G} \rho_{V}(g) \circ \psi \circ \rho_{V}\left(g^{-1}\right)=\frac{\operatorname{Tr}(\psi)}{n} \cdot \mathrm{Id} V .
$$

Proof: Since $\rho_{V}$ and $\rho_{W}$ are irreducible, the associated $K G$-modules are simple. Moreover, by Lemma 5.4, both in (a) and (b) the map $\tilde{\psi}$ is $K G$-linear. Therefore Schur's Lemma yields:

(a) $\tilde{\psi}=0$ since $V \not W$.

(b) $\widetilde{\psi}=\lambda \cdot \mathrm{Id}_{V}$ for some scalar $\lambda \in K$. Therefore, on the one hand

$$
\operatorname{Tr}(\tilde{\psi})=\frac{1}{|G|} \sum_{g \in G} \underbrace{\operatorname{Tr}\left(\rho_{V}(g) \circ \psi \circ \rho_{V}\left(g^{-1}\right)\right)}_{=\operatorname{Tr}(\psi)}=\frac{1}{|G|}|G| \operatorname{Tr}(\psi)=\operatorname{Tr}(\psi)
$$

and on the other hand

$$
\operatorname{Tr}(\widetilde{\psi})=\operatorname{Tr}\left(\lambda \cdot \operatorname{ld}_{V}\right)=\lambda \operatorname{Tr}\left(\operatorname{ld}_{V}\right)=n \cdot \lambda,
$$

hence $\lambda=\frac{\operatorname{Tr}(\psi)}{n}$.

Next, we see that Schur's Lemma implies certain "orthogonality relations" for the entries of matrix representations. 


\section{Theorem 5.6 (SchuR's Relations)}

Assume $\operatorname{char}(K) \nmid|G|$. Let $Q: G \longrightarrow \mathrm{GL}_{n}(K)$ and $P: G \longrightarrow \mathrm{GL}_{m}(K)$ be irreducible matrix representations.

(a) If $P \nsim Q$, then $\frac{1}{|G|} \sum_{g \in G} P(g)_{r i} Q\left(g^{-1}\right)_{j s}=0$ for all $1 \leqslant r, i \leqslant m$ and all $1 \leqslant j, s \leqslant n$.

(b) If $\operatorname{char}(K) \nmid n$, then $\frac{1}{|G|} \sum_{g \in G} Q(g)_{r i} Q\left(g^{-1}\right)_{j s}=\frac{1}{n} \delta_{i j} \delta_{r s}$ for all $1 \leqslant r, i, j, s \leqslant n$.

Proof: Set $V:=K^{n}, W:=K^{m}$ and let $\rho_{V}: G \longrightarrow \mathrm{GL}(V)$ and $\rho_{W}: G \longrightarrow \mathrm{GL}(W)$ be the $K$-representations induced by $Q$ and $P$, respectively, as defined in Remark 1.2. Furthermore, consider the $K$-linear map $\psi: V \longrightarrow W$ whose matrix with respect to the standard bases of $V=K^{n}$ and $W=K^{m}$ is the elementary matrix

$$
i\left[\begin{array}{c}
\vdots \\
\ldots 1 \ldots . \\
\vdots \\
j
\end{array}\right]=: E_{i j} \in M_{m \times n}(K)
$$

(i.e. the unique nonzero entry of $E_{i j}$ is its $(i, j)$-entry).

(a) By Proposition 5.5(a),

$$
\widetilde{\psi}=\frac{1}{|G|} \sum_{g \in G} \rho_{W}(g) \circ \psi \circ \rho_{V}\left(g^{-1}\right)=0
$$

because $P \nsim Q$, and hence $\rho_{V} \nsim \rho_{W}$. In particular the $(r, s)$-entry of the matrix of $\tilde{\psi}$ with respect to the standard bases of $V=K^{n}$ and $W=K^{m}$ is zero. Thus,

$$
0=\frac{1}{|G|} \sum_{g \in G}\left[P(g) E_{i j} Q\left(g^{-1}\right)\right]_{r s}=\frac{1}{|G|} \sum_{g \in G} P(g)_{r i} \cdot 1 \cdot Q\left(g^{-1}\right)_{j s}
$$

because the unique nonzero entry of the matrix $E_{i j}$ is its $(i, j)$-entry.

(b) Now we assume that $P=Q$, and hence $n=m, V=W, \rho_{V}=\rho_{W}$. Then by Proposition 5.5(b),

$$
\widetilde{\psi}:=\frac{1}{|G|} \sum_{g \in G} \rho_{V}(g) \circ \psi \circ \rho_{V}\left(g^{-1}\right)=\frac{\operatorname{Tr}(\psi)}{n} \cdot \operatorname{ld}_{V}= \begin{cases}\frac{1}{n} \cdot \operatorname{ld} d_{V} & \text { if } i=j, \\ 0 & \text { if } i \neq j .\end{cases}
$$

Therefore the $(r, s)$-entry of the matrix of $\widetilde{\psi}$ with respect to the standard basis of $V=K^{n}$ is

$$
\frac{1}{|G|} \sum_{g \in G}\left[Q(g) E_{i j} Q\left(g^{-1}\right)\right]_{r s}= \begin{cases}\left(\frac{1}{n} \cdot \mid \mathrm{d}_{V}\right)_{r s} & \text { if } i=j, \\ 0 & \text { if } i \neq j .\end{cases}
$$

Again, because the unique nonzero entry of the matrix $E_{i j}$ is its $(i, j)$-entry, it follows that

$$
\frac{1}{|G|} \sum_{g \in G} Q(g)_{r i} Q\left(g^{-1}\right)_{j s}=\frac{1}{n} \delta_{i j} \delta_{r s} .
$$

\section{$6 \quad \mathbb{C}$-Representations of Finite Abelian Groups}

In this section we give an immediate application of Schur's Lemma encoding the representation theory of finite abelian groups over the field $\mathbb{C}$ of complex numbers. 


\section{Proposition 6.1}

If $G$ is a finite abelian group, then any simple $\mathbb{C} G$-module has dimension 1.

(Equivalently, any irreducible $\mathbb{C}$-representation of $G$ has degree 1.)

Proof: Let $V$ be a simple $\mathbb{C} G$-module, and let $\rho_{V}: G \longrightarrow G L(V)$ be the associated $\mathbb{C}$-representation (i.e. as given by Proposition 4.3).

Claim: any $\mathbb{C}$-subspace of $V$ is in fact a $\mathbb{C} G$-submodule.

Proof: Fix $g \in G$ and consider $\rho_{V}(g)$. By definition $\rho_{V}(g) \in \mathrm{GL}(V)$, hence it is a $\mathbb{C}$-linear endomorphism of $V$. We claim that it is in fact $\mathbb{C} G$-linear. Indeed, as $G$ is abelian, $\forall h \in G, \forall v \in V$ we have

$$
\begin{aligned}
\rho_{V}(g)(h \cdot v)=\rho_{V}(g)\left(\rho_{V}(h)(v)\right) & =\left[\rho_{V}(g) \rho_{V}(h)\right](v) \\
& =\left[\rho_{V}(g h)\right](v) \\
& =\left[\rho_{V}(h g)\right](v) \\
& =\left[\rho_{V}(h) \rho_{V}(g)\right](v) \\
& =\rho_{V}(h)\left(\rho_{V}(g)(v)\right) \\
& =h \cdot\left(\rho_{V}(g)(v)\right)
\end{aligned}
$$

and it follows from Remark 4.4 that $\rho_{V}(g)$ is $\mathbb{C} G$-linear. Now, because $\mathbb{C}$ is algebraically closed, by part (b) of Schur's Lemma, there exists $\lambda_{g} \in \mathbb{C}$ (depending on $g$ ) such that

$$
\rho_{V}(g)=\lambda_{g} \cdot \mathrm{Id}_{V} .
$$

As this holds for every $g \in G$, it follows that any $\mathbb{C}$-subspace of $V$ is $G$-invariant, which in terms of $\mathbb{C} G$-modules means that any $\mathbb{C}$-subspace of $V$ is a $\mathbb{C} G$-submodule of $V$.

To conclude, as $V$ is simple, we deduce from the Claim that the $\mathbb{C}$-dimension of $V$ must be equal to 1 .

\section{Theorem 6.2 (Diagonalisation Theorem)}

Let $\rho: G \longrightarrow \mathrm{GL}(V)$ be a $\mathbb{C}$-representation of an arbitrary finite group $G$. Fix $g \in G$. Then, there exists an ordered $\mathbb{C}$-basis $B$ of $V$ with respect to which

$$
(\rho(g))_{B}=\left[\begin{array}{ccccc}
\varepsilon_{1} & 0 & \ldots & \ldots & 0 \\
0 & \varepsilon_{2} & \ddots & & \\
\vdots & \ddots & \ddots & \ddots & \ddots \\
\vdots & \ddots & \ddots & \ddots & \ddots \\
\vdots & & \ddots & \ddots & 0 \\
0 & \ldots & \cdots & 0 & \varepsilon_{n}
\end{array}\right],
$$

where $n:=\operatorname{dim}_{\mathbb{C}}(V)$ and each $\varepsilon_{i}(1 \leqslant i \leqslant n)$ is an $o(g)$-th root of unity in $\mathbb{C}$.

Proof: Consider the restriction of $\rho$ to the cyclic subgroup generated by $g$, that is the representation

$$
\left.\rho\right|_{\langle g\rangle}:\langle g\rangle \longrightarrow \operatorname{GL}(V) \text {. }
$$

By Corollary 3.6 to Maschke's Theorem, we can decompose the representation $\left.\rho(g)\right|_{\langle g\rangle}$ into a direct sum of irreducible $\mathbb{C}$-representations, say

$$
\left.\rho\right|_{\langle g\rangle}=\rho_{V_{1}} \oplus \cdots \oplus \rho_{V_{n}},
$$

where $V_{1}, \ldots, V_{n} \subseteq V$ are $\langle g\rangle$-invariant. Since $\langle g\rangle$ is abelian $\operatorname{dim}_{\mathbb{C}}\left(V_{i}\right)=1$ for each $1 \leqslant i \leqslant n$ by Proposition 6.1. Now, if for each $1 \leqslant i \leqslant n$ we choose a $\mathbb{C}$-basis $\left\{x_{i}\right\}$ of $V_{i}$, then there exist $\varepsilon_{i} \in \mathbb{C}$ 
$(1 \leqslant i \leqslant n)$ such that $\rho_{V_{i}}(g)=\varepsilon_{i}$ and $B:=\left(x_{1}, \ldots, x_{n}\right)$ is a $\mathbb{C}$-basis of $V$ such that

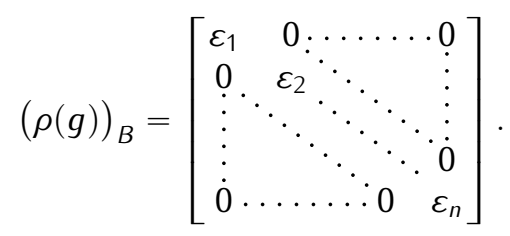

Finally, as $g^{o(g)}=1_{G}$, it follows that for each $1 \leqslant i \leqslant n$,

$$
\varepsilon_{i}^{o(g)}=\rho_{V_{i}}(g)^{o(g)}=\rho_{V_{i}}\left(g^{o(g)}\right)=\rho_{V_{i}}\left(1_{G}\right)=1_{\mathbb{C}}
$$

and hence $\varepsilon_{i}$ is an $o(g)$-th root of unity.

\section{Scholium 6.3}

If $\rho: G \longrightarrow G L(V)$ is a $\mathbb{C}$-representation of a finite abelian group, then the $\mathbb{C}$-endomorphisms $\rho(g): V \longrightarrow V$ with $g$ running through $G$ are simultaneously diagonalisable.

Proof: Same argument as in the previous proof, where we may replace " $\langle g\rangle$ " with the whole of $G$. 
We now introduce the concept of a character of a finite group. These are functions $\chi: G \longrightarrow \mathbb{C}$, obtained from the representations of the group $G$ by taking traces. Characters have many remarkable properties, and they are the fundamental tools for performing computations in representation theory. They encode a lot of information about the group itself and about its representations in a more compact and efficient manner.

Notation: throughout this chapter, unless otherwise specified, we let:

- $G$ denote a finite group;

. $K:=\mathbb{C}$ be the field of complex numbers; and

- $V$ denote a $\mathbb{C}$-vector space such that $\operatorname{dim}_{\mathbb{C}}(V)<\infty$.

In general, unless otherwise stated, all groups considered are assumed to be finite and all $\mathbb{C}$-vector spaces / modules over the group algebra considered are assumed to be finite-dimensional.

\section{Characters}

\section{Definition 7.1 (Character, linear character)}

Let $\rho_{V}: G \longrightarrow \mathrm{GL}(V)$ be a $\mathbb{C}$-representation. The character of $\rho_{V}$ is the $\mathbb{C}$-valued function

$$
\begin{aligned}
\chi_{V}: \quad G & \longrightarrow \mathbb{C} \\
g & \mapsto \chi_{V}(g):=\operatorname{Tr}\left(\rho_{V}(g)\right) .
\end{aligned}
$$

We also say that $\rho_{V}$ (or the $\mathbb{C} G$-module $V$ ) affords the character $\chi_{V}$. If the degree of $\rho_{V}$ is one, then $\chi_{V}$ is called a linear character.

\section{Remark 7.2}

(a) Again, we allow ourselves to transport terminology from representations to characters. For example, if $\rho_{V}$ is irreducible (faithful,...), then the character $\chi_{V}$ is also called irreducible (faithful,...).

With this terminology, it makes sense to $\operatorname{let} \operatorname{Irr}(G)$ denote the set of irreducible characters of $G$.

(b) Recall that in linear algebra (see GDM) the trace of a linear endomorphism $\varphi$ may be con- 
cretely computed by taking the trace of the matrix of $\varphi$ in a chosen basis of the vector space, and this is independent of the choice of the basis.

Thus to compute characters: choose an ordered basis $B$ of $V$ and obtain $\forall g \in G$ :

$$
\chi_{V}(g)=\operatorname{Tr}\left(\rho_{V}(g)\right)=\operatorname{Tr}\left(\left(\rho_{V}(g)\right)_{B}\right)
$$

(c) For a matrix representation $R: G \longrightarrow \mathrm{GL}_{n}(\mathbb{C})$, the character of $R$ is then

$$
\begin{aligned}
\chi_{R}: \quad G & \longrightarrow \mathbb{C} \\
g & \mapsto \chi_{R}(g):=\operatorname{Tr}(R(g)) .
\end{aligned}
$$

\section{Example 3}

The character of the trivial representation of $G$ is the function $1_{G}: G \longrightarrow \mathbb{C}, g \mapsto 1$ and is called the trivial character of $G$.

\section{Lemma 7.3}

Equivalent representations have the same character.

Proof: If $\rho_{V}: G \longrightarrow \mathrm{GL}(V)$ and $\rho_{W}: G \longrightarrow \mathrm{GL}(W)$ are two $\mathbb{C}$-representations, and $\alpha: V \longrightarrow W$ is an isomorphism of representations, then

$$
\rho_{W}(g)=\alpha \circ \rho_{V}(g) \circ \alpha^{-1} \quad \forall g \in G .
$$

Now, by the properties of the trace (GDM) for two $\mathbb{C}$-endomorphisms $\beta, \gamma$ of $V$ we have $\operatorname{Tr}(\beta \circ \gamma)=$ $\operatorname{Tr}(\gamma \circ \beta)$, hence for every $g \in G$ we have

$$
\chi_{W}(g)=\operatorname{Tr}\left(\rho_{W}(g)\right)=\operatorname{Tr}\left(\alpha \circ \rho_{V}(g) \circ \alpha^{-1}\right)=\operatorname{Tr}(\rho_{V}(g) \circ \underbrace{\alpha^{-1} \circ \alpha}_{=\mid d_{V}})=\operatorname{Tr}\left(\rho_{V}(g)\right)=\chi_{V}(g) .
$$

\section{Properties 7.4 (Elementary properties)}

Let $\rho_{V}: G \longrightarrow G L(V)$ be a $\mathbb{C}$-representation and let $g \in G$. Then the following assertions hold:

(a) $\chi_{V}\left(1_{G}\right)=\operatorname{dim}_{\mathbb{C}} V$;

(b) $\chi_{V}(g)=\varepsilon_{1}+\ldots+\varepsilon_{n}$, where $\varepsilon_{1}, \ldots, \varepsilon_{n}$ are $o(g)$-th roots of unity in $\mathbb{C}$ and $n=\operatorname{dim}_{\mathbb{C}} V$;

(c) $\left|\chi_{V}(g)\right| \leqslant \chi_{V}\left(1_{G}\right)$;

(d) $\chi_{V}\left(g^{-1}\right)=\overline{\chi_{V}(g)}$;

(e) if $\rho_{V}=\rho_{V_{1}} \oplus \rho_{V_{2}}$ is the direct sum of two subrepresentations, then $\chi_{V}=\chi_{V_{1}}+\chi_{V_{2}}$.

\section{Proof:}

(a) $\rho_{V}\left(1_{G}\right)=\operatorname{Id}_{V}$ because representations are group homomorphisms, hence $\chi_{V}\left(1_{G}\right)=\operatorname{dim}_{\mathbb{C}} V$.

(b) This follows directly from the diagonalisation theorem (Theorem 6.2).

(c) By (b) we have $\chi_{V}(g)=\varepsilon_{1}+\ldots+\varepsilon_{n}$, where $\varepsilon_{1}, \ldots, \varepsilon_{n}$ are roots of unity in $\mathbb{C}$. Hence, applying the triangle inequality repeatedly, we obtain that

$$
\left|\chi_{V}(g)\right|=\left|\varepsilon_{1}+\ldots+\varepsilon_{n}\right| \leqslant \underbrace{\left|\varepsilon_{1}\right|}_{=1}+\ldots+\underbrace{\left|\varepsilon_{n}\right|}_{=1}=\operatorname{dim}_{\mathbb{C}} V \stackrel{(\mathrm{a})}{=} \chi_{V}\left(1_{G}\right) .
$$


(d) Again by the diagonalisation theorem, there exists an ordered $\mathbb{C}$-basis $B$ of $V$ and $o(g)$-th roots of unity $\varepsilon_{1}, \ldots, \varepsilon_{n} \in \mathbb{C}$ such that

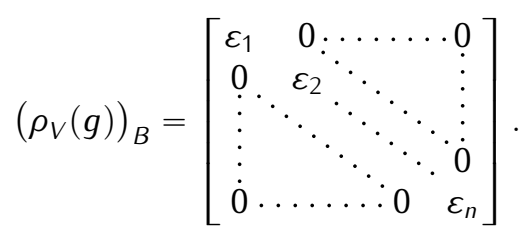

Therefore

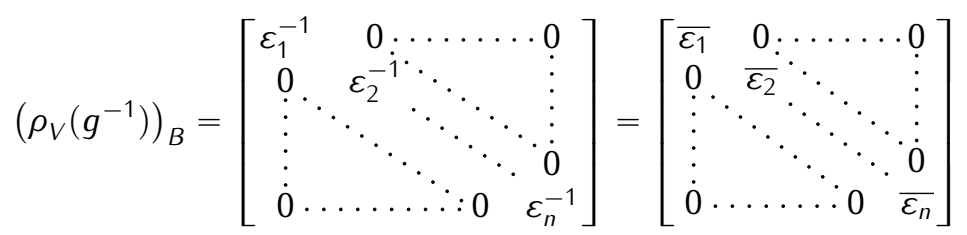

and it follows that $\chi_{V}\left(g^{-1}\right)=\overline{\varepsilon_{1}}+\ldots+\overline{\varepsilon_{n}}=\overline{\varepsilon_{1}+\ldots+\varepsilon_{n}}=\overline{\chi_{V}(g)}$.

(e) For $i=1,2$ let $B_{i}$ be an ordered $\mathbb{C}$-basis of $V_{i}$ and consider the $\mathbb{C}$-basis $B:=B_{1} \sqcup B_{2}$ of $V$. Then, by Remark 3.2 for every $g \in G$ we have

$$
\left(\rho_{V}(g)\right)_{B}=\left[\begin{array}{c|c}
\left(\rho_{W_{1}}(g)\right)_{B_{1}} & 0 \\
\hline 0 & \left(\rho_{W_{2}}(g)\right)_{B_{2}}
\end{array}\right]
$$

hence $\chi_{V}(g)=\operatorname{Tr}\left(\rho_{V}(g)\right)=\operatorname{Tr}\left(\rho_{V_{1}}(g)\right)+\operatorname{Tr}\left(\rho_{V_{2}}(g)\right)=\chi_{V_{1}}(g)+\chi_{V_{2}}(g)$.

\section{Corollary 7.5}

Any character is a sum of irreducible characters.

Proof: By Corollary 3.6 to Maschke's theorem, any $\mathbb{C}$-representation can be written as the direct sum of irreducible subrepresentations. Thus the claim follows from Properties 7.4(e).

\section{Notation 7.6}

Recall from group theory (Einfürung in die Algebra) that a group G acts on itself by conjugation via

$$
\begin{array}{ll}
G \times G & \longrightarrow G \\
(g, x) & \mapsto g \times g^{-1}=: g_{X} .
\end{array}
$$

The orbits of this action are the conjugacy classes of $G$, we denote them by $[x]:=\left\{g_{X} \mid g \in G\right\}$, and we write $C(G):=\{[x] \mid x \in G\}$ for the set of all conjugacy classes of $G$.

The stabiliser of $x \in G$ is its centraliser $C_{G}(x)=\left\{g \in G \mid{ }^{g} X=x\right\}$ and the orbit-stabiliser theorem yields

$$
\left|C_{G}(x)\right|=\frac{|G|}{|[x]|} .
$$

Moreover, a function $f: G \longrightarrow \mathbb{C}$ which is constant on each conjugacy class of $G$, i.e. such that $f\left(g x g^{-1}\right)=f(x) \forall g, x \in G$, is called a class function (on $G$ ). 


\section{Lemma 7.7}

Characters are class functions.

Proof: Let $\rho_{V}: G \longrightarrow \mathrm{GL}(V)$ be a $\mathbb{C}$-representation and let $\chi_{V}$ be its character. Again, because by the properties of the trace (GDM) $\operatorname{Tr}(\beta \circ \gamma)=\operatorname{Tr}(\gamma \circ \beta)$ for all $\mathbb{C}$-endomorphisms $\beta, \gamma$ of $V$, it follows that for all $g, x \in G$ we have

$$
\begin{aligned}
\chi_{V}\left(g x g^{-1}\right)=\operatorname{Tr}\left(\rho_{V}\left(g x g^{-1}\right)\right) & =\operatorname{Tr}\left(\rho_{V}(g) \rho_{V}(x) \rho_{V}(g)^{-1}\right) \\
& =\operatorname{Tr}(\rho_{V}(x) \underbrace{\rho_{V}(g) \rho_{V}(g)^{-1}}_{=\mathrm{ld}_{V}})=\operatorname{Tr}\left(\rho_{V}(x)\right)=\chi_{V}(x) .
\end{aligned}
$$

\section{Exercise 7.8 (Exercise 9, Sheet 3)}

Let $\rho_{V}: G \longrightarrow \mathrm{GL}(V)$ be a $\mathbb{C}$-representation and let $\chi_{V}$ be its character. Prove the following statements.

(a) If $g \in G$ is conjugate to $g^{-1}$, then $\chi_{V}(g) \in \mathbb{R}$.

(b) If $g \in G$ is an element of order 2 , then $\chi_{V}(g) \in \mathbb{Z}$ and $\chi_{V}(g) \equiv \chi_{V}(1)(\bmod 2)$.

Exercise 7.9 (The dual representation / the dual character [Exercise 10, Sheet 3])

Let $\rho_{V}: G \longrightarrow \mathrm{GL}(V)$ be a $\mathbb{C}$-representation.

(a) Prove that:

(i) the dual space $V^{*}:=\operatorname{Hom}_{\mathbb{C}}(V, \mathbb{C})$ is endowed with the structure of a $\mathbb{C} G$-module via

$$
\begin{array}{ccc}
G \times V^{*} & \longrightarrow & V^{*} \\
(g, f) & \mapsto & g . f
\end{array}
$$

where $(g . f)(v):=f\left(g^{-1} v\right) \forall v \in V$;

(ii) the character of the associated $\mathbb{C}$-representation $\rho_{V^{*}}$ is then $\chi_{V^{*}}=\overline{\chi_{V}}$; and

(iii) if $\rho_{V}$ decomposes as a direct sum $\rho_{V_{1}} \oplus \rho_{V_{2}}$ of two subrepresentations, then $\rho_{V^{*}}=\rho_{V_{1}^{*}} \oplus \rho_{V_{2}^{*}}$.

(b) Determine the duals of the 3 irreducible representations of $S_{3}$ given in Example 2(d).

\section{Orthogonality of Characters}

We are now going to make use of results from the linear algebra (GDM) on the $\mathbb{C}$-vector space of $\mathbb{C}$-valued functions on $G$ in order to develop further fundamental properties of characters.

\section{Notation 8.1}

We let $\mathcal{F}(G, \mathbb{C}):=\{f: G \longrightarrow \mathbb{C} \mid f$ function $\}$ denote the $\mathbb{C}$-vector space of $\mathbb{C}$-valued functions on $G$. Clearly $\operatorname{dim}_{\mathbb{C}} \mathcal{F}(G, \mathbb{C})=|G|$ because $\left\{\delta_{g}: G \longrightarrow \mathbb{C}, h \mapsto \delta_{g h} \mid g \in G\right\}$ is a $\mathbb{C}$-basis (see GDM). Set $\mathcal{C l}(G):=\{f \in \mathcal{F}(G, \mathbb{C}) \mid f$ is a class function $\}$. This is clearly a $\mathbb{C}$-subspace of $\mathcal{F}(G, \mathbb{C})$, called the space of class functions on $G$. 


\section{Exercise 8.2 (Exercise 11, Sheet 3)}

Find a $\mathbb{C}$-basis of $\mathcal{C l}(G)$ and deduce that $\operatorname{dim}_{\mathbb{C}} \mathcal{C l}(G)=|C(G)|$.

\section{Proposition 8.3}

The binary operation

$$
\begin{aligned}
\langle,\rangle_{G}: \mathcal{F}(G, \mathbb{C}) \times \mathcal{F}(G, \mathbb{C}) & \longrightarrow \mathbb{C} \\
\left(f_{1}, f_{2}\right) & \mapsto\left\langle f_{1}, f_{2}\right\rangle_{G}:=\frac{1}{|G|} \sum_{g \in G} f_{1}(g) \overline{f_{2}(g)}
\end{aligned}
$$

is a scalar product on $\mathcal{F}(G, \mathbb{C})$.

Proof: It is straightforward to check that $\left\langle_{,}\right\rangle_{G}$ is sesquilinear and Hermitian (Exercise 11, Sheet 3); it is positive definite because for every $f \in \mathcal{F}(G, \mathbb{C})$,

$$
\langle f, f\rangle=\frac{1}{|G|} \sum_{g \in G} f(g) \overline{f(g)}=\frac{1}{|G|} \sum_{g \in G} \underbrace{|f(g)|^{2}}_{\in \mathbb{R} \geqslant 0} \geqslant 0
$$

and moreover $\langle f, f\rangle=0$ if and only if $f=0$.

\section{Remark 8.4}

Obviously, the scalar product $\langle,\rangle_{G}$ restricts to a scalar product on $\mathcal{C l}(G)$. Moreover, if $f_{2}$ is a character of $G$, then by Property 7.4(d) we can write

$$
\left\langle f_{1}, f_{2}\right\rangle_{G}=\frac{1}{|G|} \sum_{g \in G} f_{1}(g) \overline{f_{2}(g)}=\frac{1}{|G|} \sum_{g \in G} f_{1}(g) f_{2}\left(g^{-1}\right) .
$$

The next theorem is the third key result of this lecture. It tells us that the irreducible characters of a finite group form an orthonormal system in $\mathcal{C l}(G)$ with respect to the scalar product $\langle,\rangle_{G}$.

\section{Theorem 8.5 (1st Orthogonality Relations)}

If $\rho_{V}: G \longrightarrow \mathrm{GL}(V)$ and $\rho_{W}: G \longrightarrow \mathrm{GL}(W)$ are two irreducible $\mathbb{C}$-representations with characters $\chi_{V}$ and $\chi_{W}$ respectively, then

$$
\left\langle\chi_{V}, \chi_{W}\right\rangle_{G}=\frac{1}{|G|} \sum_{g \in G} \chi_{V}(g) \chi_{W}\left(g^{-1}\right)= \begin{cases}1 & \text { if } \rho_{V} \sim \rho_{W} . \\ 0 & \text { if } \rho_{V} \nsim \rho_{W} .\end{cases}
$$

Proof: Choose ordered $\mathbb{C}$-bases $E:=\left(e_{1}, \ldots, e_{n}\right)$ and $F:=\left(f_{1}, \ldots, f_{m}\right)$ of $V$ and $W$ respectively. Then for each $g \in G$ write $Q(g):=\left(\rho_{V}(g)\right)_{E}$ and $P(g):=\left(\rho_{W}(g)\right)_{F}$.

If $\rho_{V} \nsim \rho_{W}$ compute

$$
\begin{aligned}
\left\langle\chi_{V}, \chi_{W}\right\rangle_{G}=\frac{1}{|G|} \sum_{g \in G} \chi_{V}(g) \chi_{W}\left(g^{-1}\right) & =\frac{1}{|G|} \sum_{g \in G} \operatorname{Tr}(Q(g)) \operatorname{Tr}\left(P\left(g^{-1}\right)\right) \\
& =\frac{1}{|G|} \sum_{g \in G}\left(\sum_{i=1}^{n} Q(g)_{i i}\right)\left(\sum_{j=1}^{m} P\left(g^{-1}\right)_{j j}\right) \\
& =\sum_{i=1}^{n} \sum_{j=1}^{m} \underbrace{\frac{1}{|G|} \sum_{g \in G} Q(g)_{i i} P\left(g^{-1}\right)_{j j}}_{=0 \text { by (a) of Schur's Relations }}=0
\end{aligned}
$$


and similarly if $W=V$, then $P=Q$ and

$$
\left\langle\chi_{V}, \chi_{V}\right\rangle_{G}=\sum_{i=1}^{n} \sum_{j=1}^{m} \underbrace{\frac{1}{|G|} \sum_{g \in G} Q(g)_{i i} Q\left(g^{-1}\right)_{j j}}_{=\frac{1}{n} \delta_{i j} \delta_{i j} \text { by (b) of Schur's Relations }}=\sum_{i=1}^{n} \frac{1}{n}=1 .
$$

\section{Consequences of the 1 st Orthogonality Relations}

In this section we use the 1st Orthogonality Relations in order to deduce a series of fundamental properties of the (irreducible) characters of finite groups.

\section{Corollary 9.1 (Linear independence)}

The irreducible characters of $G$ are $\mathbb{C}$-linearly independent.

Proof: Assume $\sum_{i=1}^{s} \lambda_{i} \chi_{i}=0$, where $\chi_{1}, \ldots, \chi_{s}$ are pairwise distinct irreducible characters of $G, \lambda_{1}, \ldots, \lambda_{s} \in$ $\mathbb{C}$ and $s \in \mathbb{Z}_{>0}$. Then the 1 st Orthogonality Relations yield

$$
0=\left\langle\sum_{i=1}^{s} \lambda_{i} \chi_{i}, \chi_{j}\right\rangle_{G}=\sum_{i=1}^{s} \lambda_{i} \underbrace{\left\langle\chi_{i}, \chi_{j}\right\rangle_{G}}_{=\delta_{i j}}=\lambda_{j}
$$

for each $1 \leqslant j \leqslant s$. The claim follows.

\section{Corollary 9.2 (Finiteness)}

There are at most $|C(G)|$ irreducible characters of $G$. In particular, there are only a finite number of them.

Proof: By Corollary 9.1 the irreducible characters of $G$ are $\mathbb{C}$-linearly independent. By Lemma 7.7 irrreducible characters are elements of the $\mathbb{C}$-vector space $\mathcal{C l}(G)$. Therefore there exists at most $\operatorname{dim}_{\mathbb{C}} \mathcal{C l}(G)=$ $|C(G)|<\infty$ of them.

\section{Corollary 9.3 (Multiplicities)}

Let $\rho_{V}: G \longrightarrow \mathrm{GL}(V)$ be a $\mathbb{C}$-representation and let $\rho_{V}=\rho_{V_{1}} \oplus \cdots \oplus \rho_{V_{s}}$ be a decomposition of $\rho_{V}$ into irreducible subrepresentations. Then the following assertions hold.

(a) If $\rho_{W}: G \longrightarrow \mathrm{GL}(W)$ is an irreducible $\mathbb{C}$-representation of $G$, then the multiplicity of $\rho_{W}$ in $\rho_{V_{1}} \oplus \cdots \oplus \rho_{V_{S}}$ is equal to $\left\langle\chi_{V}, \chi_{W}\right\rangle_{G}$.

(b) This multiplicity is independent of the choice of the chosen decomposition of $\rho_{V}$ into irreducible subrepresentations.

Proof: (a) We may assume that we have chosen the labelling such that

$$
\rho_{V}=\rho_{V_{1}} \oplus \cdots \oplus \rho_{V_{l}} \oplus \rho_{V_{l+1}} \oplus \cdots \oplus \rho_{V_{s}}
$$


where $\rho_{V_{i}} \sim \rho_{W} \forall 1 \leqslant i \leqslant l$ and $\rho_{V_{j}} \nsim \rho_{W} \forall l+1 \leqslant j \leqslant s$. Thus $\chi_{V_{i}}=\chi_{W} \forall 1 \leqslant i \leqslant l$ by Lemma 7.3. Therefore the 1st Orthogonality Relations yield

$$
\left\langle\chi_{V}, \chi_{W}\right\rangle_{G}=\sum_{i=1}^{l}\left\langle\chi_{V_{i}}, \chi_{W}\right\rangle_{G}+\sum_{j=l+1}^{s}\left\langle\chi_{V_{j}}, \chi_{W}\right\rangle_{G}=\sum_{i=1}^{l} \underbrace{\left\langle\chi_{W}, \chi_{W}\right\rangle_{G}}_{=1}+\sum_{j=l+1}^{s} \underbrace{\left\langle\chi_{v_{j}}, \chi_{W}\right\rangle_{G}}_{=0}=l .
$$

(b) Obvious, since $\left\langle\chi_{V}, \chi_{W}\right\rangle_{G}$ depends only on $V$ and $W$, but not on the chosen decomposition.

We can now prove that the converse of Lemma 7.3 holds.

\section{Corollary 9.4 (Equality of characters)}

Let $\rho_{V}: G \longrightarrow \mathrm{GL}(V)$ and $\rho_{W}: G \longrightarrow \mathrm{GL}(W)$ be $\mathbb{C}$-representations with characters $\chi_{V}$ and $\chi_{W}$ respectively. Then:

$$
\chi_{V}=\chi_{W} \quad \Leftrightarrow \quad \rho_{V} \sim \rho_{W}
$$

Proof: " $\Leftarrow$ ": The sufficient condition is the statement of Lemma 7.3.

$" \Rightarrow "$ : To prove the necessary condition decompose $\rho_{V}$ and $\rho_{W}$ into direct sums of irreducible subrepresentations

$$
\begin{aligned}
\rho_{V} & =\underbrace{\rho_{V_{1,1}} \oplus \cdots \oplus \rho_{V_{1, m_{1}}}}_{\text {all } \sim \rho_{V_{1}}} \oplus \cdots \oplus \underbrace{\rho_{V_{s, 1}} \oplus \cdots \oplus \rho_{V_{s, m_{s}}}}_{\text {all } \sim \rho_{V_{s}}}, \\
\rho_{W} & =\underbrace{\rho_{W_{1,1}} \oplus \cdots \oplus \rho_{W_{1, \rho_{1}}}}_{\text {all } \sim \rho_{V_{1}}} \oplus \cdots \oplus \underbrace{\rho_{W_{s, 1}} \oplus \cdots \oplus \rho_{W_{s, p_{s}}}}_{\text {all } \sim \rho_{V_{s}}},
\end{aligned}
$$

where $m_{i}, p_{i} \geqslant 0$ for all $1 \leqslant i \leqslant s$ and the $\rho_{V_{i}}$ s are pairwise non-equivalent irreducible $\mathbb{C}$ representations of $G$. (Some of the $m_{i}, p_{i}$ 's may be zero!) Now, as we assume that $\chi_{V}=\chi_{W}$, for each $1 \leqslant i \leqslant s$ Corollary 9.3 yields

$$
m_{i}=\left\langle\chi_{V}, \chi_{v_{i}}\right\rangle_{G}=\left\langle\chi_{W}, \chi_{v_{i}}\right\rangle_{G}=p_{i}
$$

hence $\rho_{V} \sim \rho_{W}$.

\section{Corollary 9.5 (Irreducibility criterion)}

A $\mathbb{C}$-representation $\rho_{V}: G \longrightarrow \mathrm{GL}(V)$ is irreducible if and only if $\left\langle\chi_{V}, \chi_{V}\right\rangle_{G}=1$.

Proof: " $\Rightarrow$ ": holds by the 1st Orthogonality Relations.

" $\Leftarrow$ : As in the previous proof, write

$$
\rho_{V}=\underbrace{\rho_{V_{1,1}} \oplus \cdots \oplus \rho_{V_{1, m_{1}}}}_{\text {all } \sim \rho_{V_{1}}} \oplus \cdots \oplus \underbrace{\rho_{V_{s, 1}} \oplus \cdots \oplus \rho_{V_{s, m_{s}}}}_{\text {all } \sim \rho_{V_{s}}},
$$

where $m_{i} \geqslant 1$ for all $1 \leqslant i \leqslant s$ and the $\rho_{V_{i}}$ s are pairwise non-equivalent irreducible $\mathbb{C}$ representations of $G$. Then, using the assumption, the sesquilinearity of the scalar product and the 1st Orthogonality Relations, we obtain that

$$
1=\left\langle\chi_{V}, \chi_{V}\right\rangle_{G}=\sum_{i=1}^{s} m_{i}^{2} \underbrace{\left\langle\chi_{v_{i}}, \chi_{V_{i}}\right\rangle_{G}}_{=1}=\sum_{i=1}^{s} m_{i}^{2} .
$$

Hence, w.l.o.g. we may assume that $m_{1}=1$ and $m_{i}=0 \forall 2 \leqslant i \leqslant s$, so that $\rho_{V}=\rho_{V_{1}}$ is irreducible. 


\section{Theorem 9.6}

The set $\operatorname{Irr}(G)$ is an orthonormal $\mathbb{C}$-basis (w.r.t. $\left.\langle,\rangle_{G}\right)$ of the $\mathbb{C}$-vector space $\mathcal{C l}(G)$ of class functions on $G$.

Proof: We already know that $\operatorname{Irr}(G)$ is a $\mathbb{C}$-linearly independent set and also that it forms an orthonormal system of $\mathcal{C l}(G)$ w.r.t. $\langle,\rangle_{G}$. Hence it remains to prove that $\operatorname{Irr}(G)$ generates $\mathcal{C l}(G)$. So let $X:=\langle\operatorname{Irr}(G)\rangle_{\mathbb{C}}$ be the $\mathbb{C}$-subspace of $\mathcal{C l}(G)$ generated by $\operatorname{Irr}(G)$. It follows that

$$
\mathcal{C l}(G)=X \oplus X^{\perp}
$$

where $X^{\perp}$ denotes the orthogonal of $X$ with respect to the scalar product $\left\langle_{1}\right\rangle_{G}$ (see GDM). Thus it is enough to prove that $X^{\perp}=0$. So let $f \in X^{\perp}$, set $\breve{f}:=\sum_{g \in G} \overline{f(g)} g \in \mathbb{C} G$ and we prove the following assertions:

(1) $\breve{f} \in Z(\mathbb{C} G)$ (the centre of $\mathbb{C} G$ ): let $h \in G$ and compute

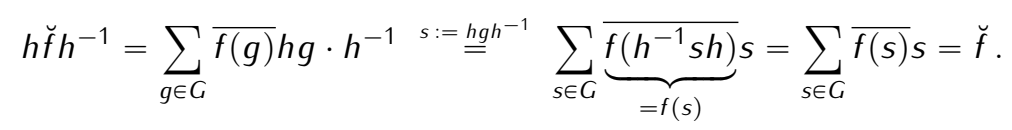

Hence $h \breve{f}=\breve{f} h$ and this equality extends by $\mathbb{C}$-linearity to the whole of $\mathbb{C} G$, so that $\breve{f} \in Z(\mathbb{C} G)$.

(2) If $V$ is a simple $\mathbb{C} G$-module with character $\chi_{V}$, then the external multiplication by $\breve{f}$ on $V$ is scalar multiplication by $\frac{|G|}{\operatorname{dim}_{C} V}\left\langle\chi_{V}, f\right\rangle_{G} \in \mathbb{C}$ : first notice that the external multiplication by $\breve{f}$ on $V$, i.e. the map

$$
\breve{f} \cdot-: V \longrightarrow V, v \mapsto \breve{f} \cdot v
$$

is $\mathbb{C} G$-linear. Indeed, for each $x \in \mathbb{C} G$ and each $v \in V$ we have

$$
\breve{f} \cdot(x \cdot v)=(\breve{f} x) \cdot v=(x \breve{f}) \cdot v=x \cdot(\breve{f} \cdot v)
$$

because $\breve{f} \in Z(\mathbb{C} G)$. Therefore, by Schur's Lemma, there exists a scalar $\lambda \in \mathbb{C}$ such that $\breve{f} \cdot-=\lambda \mathbb{I d}_{V}$. Moreover,

$$
\lambda=\frac{1}{n} \operatorname{Tr}\left(\lambda \operatorname{ld}_{V}\right)=\frac{1}{n} \operatorname{Tr}(\breve{f} \cdot-)=\frac{1}{n} \sum_{g \in G} \overline{f(g)} \underbrace{\operatorname{Tr}(\text { mult. by } g \text { on } V)}_{=\chi_{V}(g)}=\frac{1}{n} \sum_{g \in G} \overline{f(g)} \chi_{V}(g)=\frac{|G|}{n}\left\langle\chi_{V}, f\right\rangle_{G} .
$$

(3) If $V$ is a simple $\mathbb{C} G$-module with character $\chi_{V}$, then the external multiplication by $\breve{f}$ on $V$ is zero: indeed, $\left\langle\chi_{V}, f\right\rangle_{G}=0$ because $f \in X^{\perp}$ and the claim follows from (2).

(4) $f=0$ : indeed, as the external multiplication by $\breve{f}$ is zero on every simple $\mathbb{C} G$-module, it is zero on every $\mathbb{C} G$-module, because any $\mathbb{C} G$-module can be decomposed as the direct sum of simple submodules by the Corollary to Maschke's Theorem. In particular, the external multiplication by $\breve{f}$ is zero on $\mathbb{C} G$. Hence

$$
0=\breve{f} \cdot 1_{\mathbb{C} G}=\breve{f}=\sum_{g \in G} \overline{f(g)} g
$$

and we obtain that $\overline{f(g)}=0$ for each $g \in G$ because $G$ is a $\mathbb{C}$-basis of $\mathbb{C} G$. But then $f(g)=0$ for each $g \in G$ and it follows that $f=0$. 


\section{Corollary 9.7}

The number of pairwise non-equivalent irreducible characters of $G$ is equal to the number of conjugacy classes of $G$. In other words,

$$
|\operatorname{lrr}(G)|=|C(G)|
$$

Proof: By Theorem 9.6 the set $\operatorname{Irr}(G)$ is a $\mathbb{C}$-basis of the space $\mathcal{C l}(G)$ of class functions on $G$. Hence

$$
|\operatorname{lrr}(G)|=\operatorname{dim}_{\mathbb{C}} \mathcal{C l}(G)=|C(G)|
$$

where the second equality holds by Exercise 8.2.

\section{Corollary 9.8}

Let $f \in \mathcal{C l}(G)$. Then the following assertions hold:

(a) $f=\sum_{\chi \in \operatorname{Irr}(G)}\langle f, X\rangle_{G} \chi$;

(b) $\langle f, f\rangle_{G}=\sum_{X \in \operatorname{Irr}(G)}\langle f, \chi\rangle_{G}^{2}$;

(c) $f$ is a character $\Longleftrightarrow\langle f, \chi\rangle_{G} \in \mathbb{Z}_{\geqslant 0} \forall \chi \in \operatorname{Irr}(G)$; and

(d) $f \in \operatorname{Irr}(G) \Longleftrightarrow f$ is a character and $\langle f, f\rangle_{G}=1$.

Proof: (a)+(b) hold for any orthonormal basis with respect to a given scalar product (GDM).

(c) ' $\Rightarrow$ ': If $f$ is a character, then by Corollary 9.3 the complex number $\left\langle f, \chi_{i}\right\rangle_{G}$ is the multiplicity of $\chi_{i}$ as a constituent of $f$, hence a non-negative integer.

' $\Leftarrow$ ': If for each $\chi \in \operatorname{Irr}(G),\langle f, \chi\rangle_{G}=: m_{\chi} \in \mathbb{Z}_{\geqslant 0}$, then $f$ is the character of the representation

$$
\rho:=\bigoplus_{\chi \in \operatorname{lr}(G)} \bigoplus_{j=1}^{m_{\chi}} \rho(\chi)
$$

where $\rho(\chi)$ is a $\mathbb{C}$-representation affording the character $\chi$.

(d) The necessary condition is given by the 1st Orthogonality Relations. The sufficient condition follows from (b) and (c).

\section{Exercise 9.9 (Exercise 12, Sheet 3)}

Let $V$ be a $\mathbb{C} G$-module (finite dimensional) with character $\chi_{V}$. Consider the $\mathbb{C}$-subspace $V^{G}:=$ $\{v \in V \mid g \cdot v=v \forall g \in G\}$. Prove that

$$
\operatorname{dim}_{\mathbb{C}} V^{G}=\frac{1}{|G|} \sum_{g \in G} \chi_{V}(g)
$$

1. considering the scalar product of $\chi_{V}$ with the trivial character $1_{G}$;

2. seeing $V^{G}$ as the image of the projector $\pi: V \rightarrow V, V \mapsto \frac{1}{|G|} \sum_{g \in G} g \cdot v$. 


\section{The Regular Character}

Recall from Example 1(d) that a finite $G$-set $X$ induces a permutation representation

$$
\begin{aligned}
\rho_{X}: \quad G & \longrightarrow \operatorname{GL}(V) \\
g & \mapsto \rho_{X}(g): V \longrightarrow V, e_{X} \mapsto e_{g \cdot x}
\end{aligned}
$$

where $V$ is a $\mathbb{C}$-vector space with basis $\left\{e_{X} \mid x \in X\right\}$ (i.e. indexed by the set $X$ ). Given $g \in G$ write Fix $X(g):=\{x \in X \mid g \cdot x=x\}$ for the set of fixed points of $g$ on $X$.

\section{Proposition 10.1 (Character of a permutation representation)}

Let $X$ be a $G$-set and let $\chi_{X}$ denote the character of the associated permutation representation $\rho_{X}$. Then

$$
\chi_{X}(g)=\left|F_{X}(g)\right| \quad \forall g \in G .
$$

Proof: Let $g \in G$. The diagonal entries of the matrix of $\rho_{X}(g)$ expressed in the basis $B:=\left\{e_{X} \mid x \in X\right\}$ are:

$$
\left(\left(\rho_{X}(g)\right)_{B}\right)_{X X}=\left\{\begin{array}{ll}
1 & \text { if } g \cdot x=x \\
0 & \text { if } g \cdot x \neq x
\end{array} \quad \forall x \in X .\right.
$$

Hence taking traces, we get $\chi_{X}(g)=\sum_{x \in X}\left(\left(\rho_{X}(g)\right)_{B}\right)_{x X}=\left|F_{x_{X}}(g)\right|$.

For the action of $G$ on itself by left multiplication, by Example 1(d), $\rho_{X}=\rho_{\text {reg }}$ is the regular representation of $G$. In this case, we obtain the values of the regular character.

\section{Corollary 10.2 (The regular character)}

Let $\chi_{\text {reg }}$ denote the character of the regular representation $\rho_{\text {reg }}$ of $G$. Then

$$
\chi_{\mathrm{reg}}(g)= \begin{cases}|G| & \text { if } g=1_{G} \\ 0 & \text { otherwise }\end{cases}
$$

Proof: This follows immediately from Proposition 10.1 since $\operatorname{Fix}_{G}\left(1_{G}\right)=G$ and $\operatorname{Fix}_{G}(g)=\varnothing$ for every $g \in G \backslash\left\{1_{G}\right\}$.

\section{Theorem 10.3 (Decomposition of the regular representation)}

The multiplicity of an irreducible $\mathbb{C}$-representation of $G$ as a constituent of $\rho_{\text {reg }}$ equals its degree. In other words,

$$
\chi_{\text {reg }}=\sum_{\chi \in \operatorname{Irr}(G)} x(1) \chi
$$

Proof: By Corollary 9.3 we have $\chi_{\text {reg }}=\sum_{\chi \in \operatorname{Irr}(G)}\left\langle\chi_{\mathrm{reg}}, \chi\right\rangle_{G} \chi$, where for each $\chi \in \operatorname{Irr}(G)$,

$$
\left\langle\chi_{\text {reg }}, \chi\right\rangle_{G}=\frac{1}{|G|} \sum_{g \in G} \underbrace{\chi_{\text {reg }}(g)}_{\begin{array}{c}
=\delta_{1}|G| \\
\text { by Cor. 10.2 }
\end{array}} \overline{\chi(g)}=\frac{|G|}{|G|} \chi(1)=\chi(1) .
$$

The claim follows. 


\section{Remark 10.4}

In particular, the theorem tells us that each irreducible $\mathbb{C}$-representation (considered up to equivalence) occurs with multiplicity at least one in a decomposition of the regular representation into irreducible subrepresentations.

\section{Corollary 10.5 (Degree formula)}

The order of the group $G$ is given in terms of its irreducible character by the formula

$$
|G|=\sum_{x \in \operatorname{Irr}(G)} \chi(1)^{2}
$$

Proof: Evaluating the regular character at $1 \in G$ yields

$$
|G|=\chi_{\mathrm{reg}}(1)=\sum_{\chi \in \operatorname{lrr}(G)} \chi(1) \chi(1)=\sum_{\chi \in \operatorname{lrr}(G)} \chi(1)^{2} .
$$

\section{Exercise 10.6 (Exercise 13(b), Sheet 4)}

Use the degree formula to give a second proof of Proposition 6.1. In other words, prove that if $G$ is a finite abelian group, then

$$
\operatorname{Irr}(G)=\{\text { linear characters of } G\}
$$


In Chapter 3 we have proved that for any finite group $G$ the equality $|\operatorname{Irr}(G)|=|C(G)|=: r$ holds. Thus the values of the irreducible characters of $G$ can be recorded in an $r \times r$-matrix, called the character table of $G$. The entries of this matrix are related to each other in subtle manners, many of which are encapsulated in the 1st Orthogonality Relations and their consequences, as for example the degree formula. Our aim in this chapter is to develop further tools and methods to compute character tables.

Notation: throughout this chapter, unless otherwise specified, we let:

$\cdot|\operatorname{Irr}(G)|=|C(G)|=: r$;

- $G$ denote a finite group;

. $K:=\mathbb{C}$ be the field of complex numbers;

$\cdot \operatorname{Irr}(G)=\left\{\chi_{1}, \ldots, \chi_{r}\right\}$ denote the set of pairwise distinct irreducible characters of $G$;

- $C_{1}=\left[g_{1}\right], \ldots, C_{r}=\left[g_{r}\right]$ denote the conjugacy classes of $G$, where $g_{1}, \ldots, g_{r}$ is a fixed set of representatives; and

. we use the convention that $\chi_{1}=1_{G}$ and $g_{1}=1 \in G$.

In general, unless otherwise stated, all groups considered are assumed to be finite and all $\mathbb{C}$-vector spaces / modules over the group algebra considered are assumed to be finite-dimensional.

\section{The Character Table of a Finite Group}

\section{Definition 11.1 (Character table)}

The character table of $G$ is the matrix $X(G):=\left(\chi_{i}\left(g_{j}\right)\right)_{i j} \in M_{r}(\mathbb{C})$.

\section{Example 4 (The character table of a cyclic group)}

$$
\begin{array}{r}
\text { Let } G=\left\langle g \mid g^{n}=1\right\rangle \text { be cyclic of order } n \in \mathbb{Z}_{>0} . \\
\qquad \operatorname{lrr}(G)=\{\text { linear characters of } G\}
\end{array}
$$

by Proposition 6.1 and $|\operatorname{Irr}(G)|=|G|=n$. Moreover, each conjugacy class is a singleton:

$$
\forall 1 \leqslant j \leqslant r: \quad C_{j}=\left\{g_{j}\right\} \text { and we set } g_{j}:=g^{j-1} .
$$


Let $\zeta$ be a primitive $n$-th root of unity in $\mathbb{C}$, so that $\left\{\zeta^{i} \mid 1 \leqslant i \leqslant n\right\}$ are all the $n$-th roots of unity. Now, each $\chi_{i}: G \rightarrow \mathbb{C}^{\times}$is a group homomorphism and is determined by $\chi_{i}(g)$, which has to be an $n$-th root of $1_{\mathbb{C}}$. Therefore, we have $n$ possibilities for $\chi_{i}(g)$. We set

$$
\chi_{i}(g):=\zeta^{i-1} \quad \forall 1 \leqslant i \leqslant n \quad \Rightarrow \quad \chi_{i}\left(g^{j}\right)=\zeta^{(i-1) j} \quad \forall 1 \leqslant i \leqslant n, 0 \leqslant j \leqslant n-1
$$

Thus the character table of $G$ is

$$
X(G)=\left(\chi_{i}\left(g_{j}\right)\right)_{\substack{1 \leqslant i \leqslant n \\ 1 \leqslant j \leqslant n}}=\left(\chi_{i}\left(g^{j-1}\right)\right)_{\substack{1 \leqslant i \leqslant n \\ 1 \leqslant j \leqslant n}}=\left(\zeta^{(i-1)(j-1)}\right){ }_{\substack{1 \leqslant i \leqslant n \\ 1 \leqslant j \leqslant n}}
$$

which we visualise as follows:

\begin{tabular}{c|ccccc} 
& 1 & $g$ & $g^{2}$ & $\ldots$ & $g^{n-1}$ \\
\hline$\chi_{1}=1_{\mathrm{G}}$ & 1 & 1 & 1 & $\ldots$ & 1 \\
$\chi_{2}$ & 1 & $\zeta$ & $\zeta^{2}$ & $\ldots$ & $\zeta^{n-1}$ \\
$\chi_{3}$ & 1 & $\zeta^{2}$ & $\zeta^{4}$ & $\ldots$ & $\zeta^{2(n-1)}$ \\
$\ldots$ & $\ldots$ & $\ldots$ & $\ldots$ & $\ldots$ & $\ldots$ \\
$\chi_{n}$ & 1 & $\zeta^{n-1}$ & $\zeta^{2(n-1)}$ & $\ldots$ & $\zeta^{(n-1)^{2}}$
\end{tabular}

\section{Example 5 (The character table of $S_{3}$ )}

Let now $G:=S_{3}$ be the symmetric group on 3 letters. Recall from the AGS/Einführung in die Algebra that the conjugacy classes of $S_{3}$ are

$$
\begin{aligned}
& C_{1}=\{\mathrm{ld}\}, C_{2}=\left\{\left(\begin{array}{ll}
1 & 2
\end{array}\right),\left(\begin{array}{ll}
1 & 3
\end{array}\right),\left(\begin{array}{ll}
2 & 3
\end{array}\right)\right\}, C_{3}=\left\{\left(\begin{array}{lll}
1 & 2 & 3
\end{array}\right),\left(\begin{array}{lll}
1 & 3 & 2
\end{array}\right)\right\} \\
& \Rightarrow \quad r=3,\left|C_{1}\right|=1,\left|C_{2}\right|=3,\left|C_{3}\right|=2 .
\end{aligned}
$$

In Example 2(d) we have exhibited three non-equivalent irreducible matrix representations of $S_{3}$, which we denoted $\rho_{1}, \rho_{2}, \rho_{3}$. For each $1 \leqslant i \leqslant 3$ let $\chi_{i}$ be the character of $\rho_{i}$ and $n_{i}$ be its degree, so that $n_{1}=n_{2}=1$ and $n_{3}=2$. Hence

$$
n_{1}^{2}+n_{2}^{2}+n_{3}^{2}=6=|G| .
$$

Therefore, the degree formula tells us that $\rho_{1}, \rho_{2}, \rho_{3}$ are all

\begin{tabular}{|c|c|c|c|}
\hline & Id & $\left(\begin{array}{ll}1 & 2\end{array}\right)$ & $\left(\begin{array}{lll}1 & 2 & 3\end{array}\right)$ \\
\hline$\chi_{1}$ & 1 & 1 & 1 \\
\hline$\chi_{2}$ & 1 & -1 & 1 \\
\hline 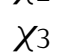 & 2 & 0 & -1 \\
\hline
\end{tabular}
the irreducible matrix representations of $S_{3}$, up to equivalence. We note that $n_{1}=n_{2}=1, n_{3}=2$ is in fact the unique solution (up to relabelling) to the equation given by the degree formula! Taking traces of the matrices in Example 2(d) yields the character table of $S_{3}$.

In the next sections we want to develop further techniques to compute character tables of finite groups, before we come back to further examples of such tables for larger groups.

\section{Exercise 11.2 (Exercise 13(c), Sheet 4)}

Compute the character table of the Klein-four group $C_{2} \times C_{2}$. 


\section{The 2nd Orthogonality Relations}

The 1st Orthogonality Relations provide us with orthogonality relations between the rows of the character table. They can be rewritten as follows in terms of matrices.

\section{Exercise 12.1 (Exercise 14, Sheet 4)}

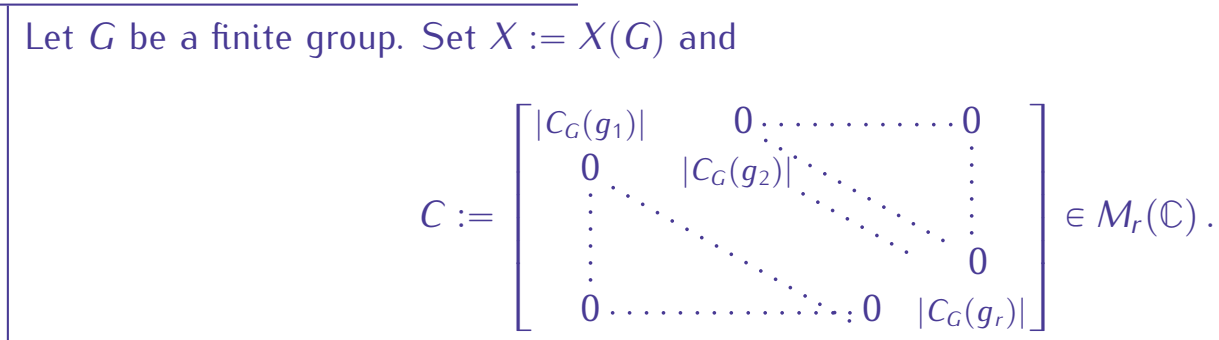

Use the Orbit-Stabiliser Theorem in order to prove that the 1st Orthogonality Relations can be rewritten under the form

$$
X C^{-1} \bar{X}^{\mathrm{Tr}}=I_{r},
$$

where $\bar{X}^{\operatorname{Tr}}$ denotes the transpose of the complex-conjugate $\bar{X}$ of the character table $X$ of $G$.

Deduce that the character table is invertible.

There are also some orthogonality relations between the columns of the character table. These can easily be deduced from the 1st Orthogonality Relations given above in terms of matrices.

\section{Theorem 12.2 (2nd Orthogonality Relations)}

With the notation of Exercise 12.1 we have

$$
X^{\operatorname{Tr}} \bar{X}=C .
$$

In other words,

$$
\sum_{\chi \in \operatorname{Irr}(G)} \chi\left(g_{i} \overline{\chi\left(g_{j}\right)}=\delta_{i j} \frac{|G|}{\left|\left[g_{i}\right]\right|}=\delta_{i j}\left|C_{G}\left(g_{i}\right)\right| \quad \forall 1 \leqslant i, j \leqslant r .\right.
$$

Proof: Taking complex conjugation of the formula given by the 1st Orthogonality Relations (Exercise 12.1) yields:

$$
X C^{-1} \bar{X}^{\operatorname{Tr}}=I_{r} \quad \Longrightarrow \quad \bar{X} C^{-1} X^{\operatorname{Tr}}=I_{r}
$$

Now, since $X$ is invertible, so are all the matrices in the above equations and hence $X^{\operatorname{Tr}}=\left(\bar{X} C^{-1}\right)^{-1}$. It follows that

$$
X^{\operatorname{Tr}} \bar{X}=\left(\bar{X} C^{-1}\right)^{-1} \bar{X}=C \bar{X}^{-1} \bar{X}=C .
$$

The second formula is now obtained by considering the entry $(i, j)$ in the above matrix equation for all $1 \leqslant i, j \leqslant r$.

\section{Exercise 12.3 (Exercise 13(a), Sheet 4)}

Prove that the degree formula can be read off from the 2nd Orthogonality Relations. 


\section{Tensor Products of Representations and Characters}

Tensor products of vector spaces and matrices are recalled/introduced in Appendix $\mathrm{C}$. We are now going to use this construction to build products of characters.

\section{Proposition 13.1}

Let $G$ and $H$ be finite groups, and let $\rho_{V}: G \longrightarrow \mathrm{GL}(V)$ and $\rho_{W}: H \longrightarrow \mathrm{GL}(W)$ be $\mathbb{C}$ representations with characters $\chi_{V}$ and $\chi_{W}$ respectively. Then

$$
\begin{aligned}
& \rho_{V} \otimes \rho_{W}: \quad \begin{array}{l}
G \times H \\
(g, h)
\end{array} \mapsto \quad \operatorname{GL}(V \otimes \mathbb{C} W) \\
&\left(\rho_{V} \otimes \rho_{W}\right)(g, h):=\rho_{V}(g) \otimes \rho_{W}(h)
\end{aligned}
$$

(where $\rho_{V}(g) \otimes \rho_{W}(h)$ is the tensor product of the $\mathbb{C}$-endomorphisms $\rho_{V}(g): V \longrightarrow V$ and $\rho_{W}(h)$ : $W \longrightarrow W$ as defined in Lemma-Definition C.4) is a $\mathbb{C}$-representation of $G \times H$, called the tensor product of $\rho_{V}$ and $\rho_{W}$, and the corresponding character, which we denote by $\chi_{V \otimes_{\mathbb{C}} W}$, is

$$
\chi_{V \otimes_{\mathbb{C}} W}=\chi_{V} \cdot \chi_{W}
$$

where $\chi_{V} \cdot \chi_{W}(g, h):=\chi_{V}(g) \cdot \chi_{W}(h) \forall(g, h) \in G \times H$.

Proof: First note that $\rho_{V} \otimes \rho_{W}$ is well-defined by Lemma-Definition C.4 and it is a group homomorphism because

$$
\begin{aligned}
\left(\rho_{V} \otimes \rho_{W}\right)\left(g_{1} g_{2}, h_{1} h_{2}\right)[v \otimes w] & =\left(\rho_{V}\left(g_{1} g_{2}\right) \otimes \rho_{W}\left(h_{1} h_{2}\right)\right)[v \otimes w] \\
& =\rho_{V}\left(g_{1} g_{2}\right)[v] \otimes \rho_{W}\left(h_{1} h_{2}\right)[w] \\
& =\rho_{V}\left(g_{1}\right) \circ \rho_{V}\left(g_{2}\right)[v] \otimes \rho_{W}\left(h_{1}\right) \circ \rho_{W}\left(h_{2}\right)[w] \\
& =\rho_{V}\left(g_{1}\right) \otimes \rho_{W}\left(h_{1}\right)\left[\rho_{V}\left(g_{2}\right)[v] \otimes \rho_{W}\left(h_{2}\right)[w]\right] \\
& =\left(\rho_{V}\left(g_{1}\right) \otimes \rho_{W}\left(h_{1}\right)\right) \circ\left(\rho_{V}\left(g_{2}\right) \otimes \rho_{W}\left(h_{2}\right)\right)[v \otimes w] \\
& =\left(\rho_{V} \otimes \rho_{W}\right)\left(g_{1}, h_{1}\right) \circ\left(\rho_{V} \otimes \rho_{W}\right)\left(g_{2}, h_{2}\right)[v \otimes w]
\end{aligned}
$$

$\forall g_{1}, g_{2} \in G, h_{1}, h_{2} \in H, v \in V, w \in W$. Furthermore, for each $g \in G$ and each $h \in H$,

$\chi_{V \otimes_{\mathbb{C}} W}(g, h)=\operatorname{Tr}\left(\left(\rho_{V} \otimes \rho_{W}\right)(g, h)\right)=\operatorname{Tr}\left(\rho_{V}(g) \otimes \rho_{W}(h)\right)=\operatorname{Tr}\left(\rho_{V}(g)\right) \cdot \operatorname{Tr}\left(\rho_{W}(h)\right)=\chi_{V}(g) \cdot \chi_{W}(h)$

by Lemma-Definition C.4, hence $\chi_{V \otimes_{\mathbb{C}} W}=\chi_{V} \cdot \chi_{W}$.

Remark 13.2

The diagonal inclusion $\iota: G \longrightarrow G \times G, g \mapsto(g, g)$ of $G$ in the product $G \times G$ is a group homomorphism with $\iota(G) \cong G$. Therefore, if $G=H$, then

$$
G \stackrel{\iota}{\longrightarrow} G \times G \stackrel{\chi_{V} \cdot \chi_{W}}{\longrightarrow} \mathbb{C}, g \mapsto(g, g) \mapsto \chi_{V}(g) \cdot \chi_{W}(g)
$$

becomes a character of $G$, which we also denote by $\chi_{V} \cdot \chi_{W}$.

\section{Corollary 13.3}

If $G$ and $H$ are finite groups, then $\operatorname{Irr}(G \times H)=\{\chi \cdot \psi \mid \chi \in \operatorname{Irr}(G), \psi \in \operatorname{Irr}(H)\}$.

Proof: [Exercise 15(c), Sheet 4]. Hint: Use Corollary 9.8(d) and the degree formula. 


\section{Exercise 13.4 (Exercise 15(a)+(b), Sheet 4)}

(a) If $\lambda, \chi \in \operatorname{Irr}(G)$ and $\lambda(1)=1$, then $\lambda \cdot \chi \in \operatorname{Irr}(G)$.

(b) The set $\{\chi \in \operatorname{Irr}(G) \mid \chi(1)=1\}$ of linear characters of a finite group $G$ forms a group for the product of characters.

\section{Normal Subgroups and Inflation}

Whenever a group homomorphism $G \longrightarrow H$ and a representation of $H$ are given, we obtain a representation of $G$ by composition. In particular, we want to apply this principle to normal subgroups $N \leqslant G$ and the corresponding quotient homomorphism, which we always denote by $\pi: G \longrightarrow G / N, g \mapsto g N$.

We will see that by this means, copies of the character tables of quotient groups of $G$ all appear in the character table of $G$. This observation, although straightforward, will allow us to fill out the character table of a group very rapidly, provided it possesses normal subgroups.

\section{Definition 14.1 (Inflation)}

Let $N \preccurlyeq G$ and let $\pi: G \longrightarrow G / N, g \mapsto g N$ be the quotient homomorphism. Given a $\mathbb{C}$ representation $\rho: G / N \longrightarrow G L(V)$, we set

$$
\operatorname{lnf}_{G / N}^{G}(\rho):=\rho \circ \pi: G \longrightarrow G L(V) .
$$

This is a $\mathbb{C}$-representation of $G$, called the inflation of $\rho$ from $G / N$ to $G$. If the character of $\rho$ is $\chi$, then we denote by $\operatorname{lnf}_{G / N}^{G}(\chi)$ the character of $\operatorname{lnf}_{G / N}^{G}(\rho)$ and call it the inflation of $\chi$ from $G / N$ to $G$.

Note that some texts also call $\operatorname{lnf}_{G / N}^{G}(\rho)\left(\right.$ resp. $\left.\operatorname{lnf}_{G / N}^{G}(\chi)\right)$ the lift of $\rho$ (resp. $\chi$ ) along $\pi$.

\section{Remark 14.2}

The values of the character $\operatorname{lnf}_{G / N}^{G}(X)$ of $G$ are obtained from those of $X$ as follows. If $g \in G$, then

$$
\operatorname{Inf}_{G / N}^{G}(\chi)(g)=\operatorname{Tr}((\rho \circ \pi)(g))=\operatorname{Tr}(\rho(g N))=\chi(g N) .
$$

\section{Exercise 14.3 (Exercise 16, Sheet 4)}

Let $N \preccurlyeq G$ and let $\rho: G / N \longrightarrow G L(V)$ be a $\mathbb{C}$-representation of $G / N$ with character $\chi$.

(a) Prove that if $\rho$ is irreducible, then so is $\operatorname{lnf}_{G / N}^{G}(\rho)$.

(b) Compute the kernel of $\operatorname{Inf}_{G / N}^{G}(\rho)$ provided that $\rho$ is faithful.

\section{Definition 14.4 (Kernel of a character)}

The kernel of a character $\chi$ of $G$ is $\operatorname{ker}(\chi):=\{g \in G \mid \chi(g)=\chi(1)\}$. 


\section{Example 6}

(a) $\chi=1_{G}$ the trivial character $\Rightarrow \operatorname{ker}(\chi)=G$.

(b) $G=\mathfrak{S}_{3}, \chi=\chi 2$ the sign character $\Rightarrow \operatorname{ker}(\chi)=C_{1} \cup C_{3}=\langle(123)\rangle$; whereas $\operatorname{ker}\left(\chi_{3}\right)=\{1\}$. (See Example 5.)

\section{Lemma 14.5}

Let $\rho: G \longrightarrow \mathrm{GL}(V)$ be a $\mathbb{C}$-representation of $G$ with character $\psi$. Then $\operatorname{ker}(\psi)=\operatorname{ker}(\rho)$, thus is a normal subgroup of $G$.

Proof: [Exercise 17(a), Sheet 5]

\section{Theorem 14.6}

Let $N \preccurlyeq G$. Then

$$
\begin{aligned}
\operatorname{Inf}_{G / N}^{G}:\{\text { characters of } G / N\} & \longrightarrow\{\text { characters } \psi \text { of } G \mid N \leqslant \operatorname{ker}(\psi)\} \\
x & \mapsto \operatorname{Inf}_{G / N}^{G}(x)
\end{aligned}
$$

is a bijection and so is its restriction to the irreducible characters

$$
\begin{aligned}
\operatorname{Inf}_{G / N}^{G}: \operatorname{Irr}(G / N) & \longrightarrow\{\psi \in \operatorname{Irr}(G) \mid N \leqslant \operatorname{ker}(\psi)\} \\
\chi & \mapsto \operatorname{Inf}_{G / N}^{G}(\chi) .
\end{aligned}
$$

Proof: First we prove that the first map is well-defined and bijective.

- Let $\chi$ be a character of $G / N$ afforded by the $\mathbb{C}$-representation $\rho: G / N \longrightarrow G L(V)$. By Remark 14.2, $N$ is in the kernel of $\operatorname{lnf}_{G / N}^{G}(\chi)$, hence the first map is well-defined.

- Now let $\psi$ be a character of $G$ with $N \leqslant \operatorname{ker}(\psi)$ and assume $\psi$ is afforded by the $\mathbb{C}$-representation $\rho: G \longrightarrow \mathrm{GL}(V)$.

$$
\begin{aligned}
& G \stackrel{\rho}{\longrightarrow} \operatorname{GL}(V) \quad \text { By Lemma } 14.5 \text { we have } \operatorname{ker}(\psi)=\operatorname{ker}(\rho) \geqslant N \text {. Therefore, by the } \\
& \pi \quad 0,-\pi \quad \text { universal property of the quotient, } \rho \text { induces a unique } \mathbb{C} \text {-representation } \\
& \approx,-{ }^{\prime} ! \tilde{\rho} \quad \tilde{\rho}: G / N \longrightarrow \operatorname{GL}(V) \text { with the property that } \tilde{\rho} \circ \pi=\rho \text {. }
\end{aligned}
$$

It follows that $\rho=\operatorname{Inf}_{G / N}^{G}(\tilde{\rho})$ and $\psi=\operatorname{Inf}_{G / N}^{G}(X)$. Thus the 1 st map is surjective. Its injectivity is clear.

The second map is well-defined by the above and Exercise 14.3(a). It is injective because it is just the restriction of the 1st map to the $\operatorname{Irr}(G / N)$, whereas it is surjective by the same argument as above as the constructed representation $\tilde{\rho}$ is clearly irreducible if $\rho$ is because $\tilde{\rho} \circ \pi=\rho$.

\section{Exercise 14.7 (Exercise 17(b), Sheet 5)}

Let $G$ be a finite group. Prove that if $N \leqslant G$, then

$$
N=\bigcap_{\substack{x \in \operatorname{lrr}(G) \\ N \subseteq \operatorname{ker}(x)}} \operatorname{ker}(\chi) .
$$


It follows immediately from the above exercise that the lattice of normal subgroups of $G$ can be read off from its character table. The theorem also implies that it can be read off from the character table, whether the group is abelian or simple.

\section{Corollary 14.8}

(a) Inflation from the abelianisation induces a bijection

$$
\operatorname{lnf}_{G / G^{\prime}}^{G}: \operatorname{Irr}\left(G / G^{\prime}\right) \longrightarrow\{\psi \in \operatorname{Irr}(G) \mid \psi(1)=1\}
$$

in particular, $G$ has precisely $\left|G: G^{\prime}\right|$ linear characters.

(b) The group $G$ is abelian if and only if all its irreducible characters are linear.

Proof: (a) First, we claim that if $\psi \in \operatorname{Irr}(G)$ is linear, then $G^{\prime}$ is in its kernel. Indeed, if $\psi(1)=1$, then $\psi: G \longrightarrow \mathbb{C}^{\times}$is a group homomorphism. Therefore, as $\mathbb{C}^{\times}$is abelian,

$$
\psi([g, h])=\psi\left(g h g^{-1} h^{-1}\right)=\psi(g) \psi(h) \psi(g)^{-1} \psi(h)^{-1}=\psi(g) \psi(g)^{-1} \psi(h) \psi(h)^{-1}=1
$$

for all $g, h \in G$, and hence $G^{\prime}=\langle[g, h] \mid g, h \in G\rangle \leqslant \operatorname{ker}(\chi)$. In addition, any irreducible character of $G / G^{\prime}$ is linear by Proposition 6.1 because $G / G^{\prime}$ is abelian. Thus Theorem 14.6 yields a bijection

$$
\operatorname{Irr}\left(G / G^{\prime}\right) \underset{\operatorname{lnf}_{G / G^{\prime}}^{\mathcal{C}}}{\sim}\left\{\psi \in \operatorname{Irr}(G) \mid G^{\prime} \leqslant \operatorname{ker}(\psi)\right\}=\{\psi \in \operatorname{Irr}(G) \mid \psi(1)=1\},
$$

as required.

(b) If $G$ is abelian, then $G / G^{\prime}=G$. Hence the claim follows from (a).

\section{Corollary 14.9}

A finite group $G$ is simple $\Longleftrightarrow \chi(g) \neq \chi(1) \forall g \in G \backslash\{1\}$ and $\forall \chi \in \operatorname{Irr}(G) \backslash\left\{1_{G}\right\}$.

Proof: [Exercise 18, Sheet 5]

\section{Exercise 14.10 (Exercise 19, Sheet 5)}

Compute the complex character table of the alternating group $A_{4}$ through the following steps:

1. Determine the conjugacy classes of $A_{4}$ (there are 4 of them) and the corresponding centraliser orders.

2. Determine the degrees of the 4 irreducible characters of $A_{4}$.

3. Determine the linear characters of $A_{4}$.

4. Determine the non-linear character of $A_{4}$ using the 2nd Orthogonality Relations.

To finish this section we show how to compute the character table of the symmetric group $S_{4}$ combining several of the techniques we have developed in this chapter. 


\section{Example 7 (The character table of $S_{4}$ )}

Again the conjugacy classes of $S_{4}$ are given by the cycle types. We fix

$$
\begin{aligned}
& C_{1}=\{\mathrm{Id}\}, C_{2}=\left[\left(\begin{array}{ll}
1 & 2
\end{array}\right)\right], C_{3}=\left[\left(\begin{array}{lll}
1 & 2 & 3
\end{array}\right)\right], C_{4}=\left[\left(\begin{array}{ll}
1 & 2
\end{array}\right)(34)\right], C_{5}=[(1234)] \\
& \Rightarrow \quad r=5,\left|C_{1}\right|=1,\left|C_{2}\right|=6,\left|C_{3}\right|=8,\left|C_{4}\right|=3,\left|C_{5}\right|=6 .
\end{aligned}
$$

Hence $|\operatorname{Irr}(G)|=|C(G)|=5$ and as always we may assume that $\chi_{1}=1_{G}$ is the trivial character.

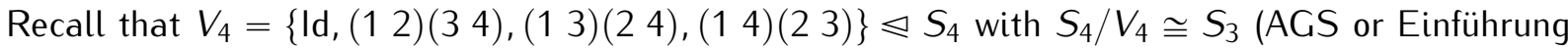
in die Algebra!). Therefore, by Theorem 14.6 we can "inflate" the character table of $S_{4} / V_{4} \cong S_{3}$ to $S_{4}$ (see Example 5 for the character table of $S_{3}$ ). This provides us with three irreducible characters

\begin{tabular}{|c|c|c|c|c|c|}
\hline \multirow{2}{*}{$\mid C_{G}\left(g_{i}\right)$} & Id & (1 2) & (1 123$)$ & $(12)(34)$ & 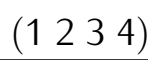 \\
\hline & 24 & 4 & 3 & 8 & 4 \\
\hline$x_{1}$ & 1 & 1 & 1 & 1 & 1 \\
\hline$x_{2}$ & 1 & -1 & 1 & 1 & -1 \\
\hline$x_{3}$ & 2 & 0 & -1 & 2 & 0 \\
\hline$\chi_{4}$ & . & . & . & . & . \\
\hline$x_{5}$ & & . & & & . \\
\hline
\end{tabular}
$\chi_{1}, x_{2}$ and $x_{3}$ of $S_{4}$ :

Here we have computed the values of $\chi_{2}$ and $\chi_{3}$ using Remark 14.2 as follows:

- Inflation preserves degrees, hence it follows from Example 5 that $\chi_{2}(\mathrm{Id})=1$ and $\chi_{3}(\mathrm{Id})=2$. (Up to relabelling!)

- As $C_{4}=\left[\left(\begin{array}{ll}1 & 2\end{array}\right)(34)\right] \subseteq V_{4},\left(\begin{array}{ll}1 & 2\end{array}\right)(34) \in \operatorname{ker}\left(\chi_{i}\right)$ for $i=2,3$ and hence $\chi_{2}\left(\left(\begin{array}{ll}1 & 2\end{array}\right)(34)\right)=1$ and $\chi_{3}\left(\left(\begin{array}{ll}1 & 2)\end{array}\right)(34)\right)=2$.

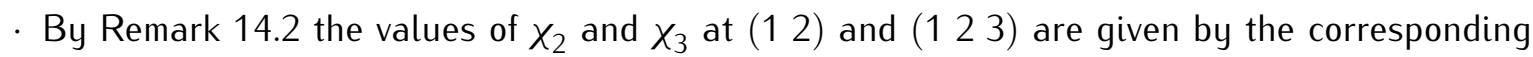
values in the character table of $S_{3}$. (Here it is enough to argue that the isomorphism between $S_{4} / V_{4}$ and $S_{3}$ must preserve orders of elements, hence also the cycle type in this case.)

- Finally, we compute that $\overline{\left(\begin{array}{llll}1 & 2 & 3\end{array}\right)}=\overline{\left(\begin{array}{lll}1 & 2\end{array}\right)} \in S_{4} / V_{4}$, hence $\chi_{i}\left(\left(\begin{array}{lll}1 & 2 & 3\end{array}\right)\right)=\chi_{i}\left(\left(\begin{array}{ll}1 & 2\end{array}\right)\right)$ for $i=2,3$.

Therefore, it remains to compute $\chi_{4}$ and $\chi_{5}$. To begin with the degree formula yields

$$
\sum_{i=1}^{5} \chi_{i}(\mathrm{Id})^{2}=24 \quad \Longrightarrow \quad \chi_{4}(\mathrm{ld})^{2}+\chi_{5}(\mathrm{Id})^{2}=18 \quad \Longrightarrow \quad \chi_{4}(\mathrm{Id})=\chi_{5}(\mathrm{Id})=3
$$

Next, the 2nd Orthogonality Relations applied to the 3rd column with itself read

$$
\left.\left.\left.\sum_{i=1}^{5} \chi_{i}\left(\left(\begin{array}{lll}
1 & 2 & 3
\end{array}\right)\right) \overline{\chi_{i}\left(\left(\begin{array}{llll}
1 & 2 & 3
\end{array}\right)\right)}=\sum_{i=1}^{5} \chi_{i}\left(\begin{array}{llll}
1 & 2 & 3
\end{array}\right)\right) \chi_{i}\left(\begin{array}{lll}
1 & 2 & 3
\end{array}\right)^{-1}\right)=\mid C_{G}\left(\begin{array}{lll}
1 & 2 & 3
\end{array}\right)\right) \mid=3
$$

hence $\left.1+1+1+\chi_{4}\left(\left(\begin{array}{lll}1 & 2 & 3\end{array}\right)\right)^{2}+\chi_{5}\left(\begin{array}{lll}1 & 2 & 3\end{array}\right)\right)^{2}=3$ and it follows that $\chi_{4}\left(\left(\begin{array}{lll}1 & 2 & 3\end{array}\right)\right)=\chi_{5}\left(\left(\begin{array}{lll}1 & 2 & 3\end{array}\right)\right)=0$. Similarly, the 2nd Orthogonality Relations applied to the 2nd column with itself / the 4th column with itself and the 5th column with itself yield that all other entries squared are equal to 1 , hence 
all other entries are \pm 1 .

The 2nd Orthogonality Relations applied to the 1 st and 2 nd columns give the 2 nd column, i.e. $\chi_{4}\left(\left(\begin{array}{ll}1 & 2\end{array}\right)\right)=1$ and $\chi_{5}\left(\left(\begin{array}{ll}1 & 2\end{array}\right)\right)=-1$ (up to swapping $\chi_{4}$ and $\left.\chi_{5}\right)$.

Then the 1 st Orthogonality Relations applied to the $3 r d$ and the 4 th row yield

$$
0=\sum_{k=1}^{5} \frac{1}{\left|C_{G}\left(g_{k}\right)\right|} \chi_{3}\left(g_{k}\right) \overline{\chi_{4}\left(g_{k}\right)}=\frac{6}{24}+\frac{1}{4} \chi_{4}((12)(34)) \Rightarrow \chi_{4}((12)(34))=-1 .
$$

Similar with the 3rd row and the 5th row: $\chi_{5}\left(\begin{array}{ll}1 & 2\end{array}\right)\left(\begin{array}{ll}3 & 4\end{array}\right)=-1$. Finally the 1 st Orthogonality Relations applied to the 1st and the 4th (resp. 5th) row yield $\left.\chi_{4}\left(\begin{array}{llll}1 & 2 & 3 & 4\end{array}\right)\right)=-1$ (resp. $\left.\chi_{5}\left(\left(\begin{array}{llll}1 & 2 & 3 & 4\end{array}\right)\right)=1\right)$. Thus the character table of $S_{4}$ is:

\begin{tabular}{|c|c|c|c|c|c|}
\hline \multirow{2}{*}{$\left|C_{G}\left(g_{i}\right)\right|$} & Id & (12) & (1 23 ) & $(12)(34)$ & $(1234)$ \\
\hline & 24 & 4 & 3 & 8 & 4 \\
\hline$\chi_{1}$ & 1 & 1 & 1 & 1 & 1 \\
\hline$x_{2}$ & 1 & -1 & 1 & 1 & -1 \\
\hline$\chi_{3}$ & 2 & 0 & -1 & 2 & 0 \\
\hline$\chi_{4}$ & 3 & 1 & 0 & -1 & -1 \\
\hline$x_{5}$ & 3 & -1 & 0 & -1 & 1 \\
\hline
\end{tabular}

\section{Remark 14.11}

Two non-isomorphic groups can have the same character table. E.g.: $Q_{8}$ and $D_{8}$, but $Q_{8} \neq D_{8}$. So the character table does not determine:

- the group up to isomorphism;

- the full lattice of subgroups;

- the orders of elements.

\section{Exercise 14.12 (Exercise 20(a), Sheet 5)}

Compute the character tables of $D_{8}$ and $Q_{8}$.

[Hint: In each case, determine the commutator subgroup and deduce that there are 4 linear characters.]

\section{Exercise 14.13 (The determinant of a representation / Exercise 20(b), Sheet 5)}

If $\rho: G \longrightarrow \mathrm{GL}(V)$ is a $\mathbb{C}$-representation of $G$ and det $: \mathrm{GL}(V) \longrightarrow \mathbb{C}^{*}$ denotes the determinant homomorphism, then we define a linear character of $G$ via

$$
\operatorname{det}_{\rho}:=\operatorname{det} \circ \rho: G \longrightarrow \mathbb{C}^{*},
$$

called the determinant of $\rho$. Prove that, although the finite groups $D_{8}$ and $Q_{8}$ have the same character table, they can be distinguished by considering the determinants of their irreducible $\mathbb{C}$-representations. 


\section{Exercise 14.14 (Exercise 21, Sheet 6)}

Prove the follwing assertions:

(a) If $G$ is a non-abelian simple group (or more generally if $G$ is perfect, i.e. $G=[G, G]$ ), then the image $\rho(G)$ of any $\mathbb{C}$-representation $\rho: G \longrightarrow \mathrm{GL}(V)$ is a subgroup of $\operatorname{SL}(V)$.

(b) No simple group $G$ has an irreducible character of degree 2.

Assume that $G$ is simple and $\rho: G \longrightarrow \mathrm{GL}_{2}(\mathbb{C})$ is an irreducible matrix representation of $G$ with character $\chi$ and proceed as follows:

1. Prove that $\rho$ is faithful and $G$ is non-abelian.

3. Determine the determinant $\operatorname{det}_{\rho}$ of $\rho$.

4. Prove that $|G|$ is even and $G$ admits an element $x$ of order 2 .

5. Prove that $\langle x\rangle \triangleleft G$ and conclude that assertion (b) holds. 
Chapter 5. Integrality and Theorems of Burnside's

The main aim of this chapter is to prove Burnside's $p^{a} q^{b}$ theorem, which provides us with a solubility criterion for finite groups of order $p^{a} q^{b}$ with $p, q$ prime numbers, which is extremely hard to prove by purely group theoretic methods. To reach this aim, we need to develop techniques involving the integrality of character values and further results of Burnside's on the vanishing of character values.

Notation: throughout this chapter, unless otherwise specified, we let:

- $G$ denote a finite group;

- $K:=\mathbb{C}$ be the field of complex numbers;

- $\operatorname{Irr}(G):=\left\{\chi_{1}, \ldots, \chi_{r}\right\}$ denote the set of pairwise distinct irreducible characters of $G$;

- $C_{1}=\left[g_{1}\right], \ldots, C_{r}=\left[g_{r}\right]$ denote the conjugacy classes of $G$, where $g_{1}, \ldots, g_{r}$ is a fixed set of representatives; and

- we use the convention that $\chi_{1}=1_{G}$ and $g_{1}=1 \in G$.

In general, unless otherwise stated, all groups considered are assumed to be finite and all $\mathbb{C}$-vector spaces / modules over the group algebra considered are assumed to be finite-dimensional.

\section{Algebraic Integers and Character Values}

First we investigate the algebraic nature of character values.

Recall: (See Appendix D for details.)

An element $b \in \mathbb{C}$ which is integral over $\mathbb{Z}$ is called an algebraic integer. In other words, $b \in \mathbb{C}$ is an algebraic integer if $b$ is a root of monic polynomial $f \in \mathbb{Z}[X]$.

Algebraic integers have the following properties:

- The integers are clearly algebraic integers.

- Roots of unity are algebraic integers, as they are roots of polynomials of the form $X^{m}-1 \in \mathbb{Z}[X]$.

- The algebraic integers form a subring of $\mathbb{C}$. In particular, sums and products of algebraic integers are again algebraic integers.

- If $b \in \mathbb{Q}$ is an algebraic integer, then $b \in \mathbb{Z}$. In other words $\{b \in \mathbb{Q} \mid b$ algebraic integer $\}=\mathbb{Z}$. 


\section{Corollary 15.1}

Character values are algebraic integers.

Proof: By the above, roots of unity are algebraic integers. Since the algebraic integers form a ring, so are sums of roots of unity. Hence the claim follows from Property 7.4(b).

\section{Central Characters}

We now extend representations/characters of finite groups to "representations/characters" of the centre of the group algebra $\mathbb{C} G$ in order to obtain further results on character values, which we will use in the next sections in order to prove Burnside's $p^{a} q^{b}$ theorem.

\section{Definition 16.1 (Class sums)}

The elements $\widehat{C}_{j}:=\sum_{g \in C_{j}} g \in \mathbb{C} G(1 \leqslant j \leqslant r)$ are called the class sums of $G$.

\section{Lemma 16.2}

The class sums $\left\{\hat{C}_{j} \mid 1 \leqslant j \leqslant r\right\}$ form a $\mathbb{C}$-basis of $Z(\mathbb{C} G)$. In other words, $Z(\mathbb{C} G)=\oplus_{j=1}^{r} \mathbb{C} \widehat{C}_{j}$.

Proof: Notice that the class sums are clearly $\mathbb{C}$-linearly independent because the group elements are.

' $\supseteq$ ': $\forall 1 \leqslant j \leqslant r$ and $\forall g \in G$, we have

$$
g \cdot \widehat{C}_{j}=g\left(g^{-1} \widehat{C}_{j} g\right)=\widehat{C}_{j} \cdot g .
$$

Extending by $\mathbb{C}$-linearity, we get $a \cdot \widehat{C}_{j}=\widehat{C}_{j} \cdot a \quad \forall 1 \leqslant j \leqslant r$ and $\forall a \in \mathbb{C} G$. Thus $\bigoplus_{j=1}^{r} \mathbb{C} \widehat{C}_{j} \subseteq Z(\mathbb{C} G)$.

' $\subseteq$ ': Let $a \in Z(\mathbb{C} G)$ and write $a=\sum_{g \in G} \lambda_{g} g$ with $\left\{\lambda_{g}\right\}_{g \in G} \in \mathbb{C}$. Since $a$ is central, for every $h \in G$, we have

$$
\sum_{g \in G} \lambda_{g} g=a=h a h^{-1}=\sum_{g \in G} \lambda_{g}\left(h g h^{-1}\right) .
$$

Comparing coefficients yield $\lambda_{g}=\lambda_{h g h^{-1}} \forall g, h \in G$. Namely, the coefficients $\lambda_{g}$ are constant on the conjugacy classes of $G$, and hence

$$
a=\sum_{j=1}^{r} \lambda_{g_{j}} \widehat{C}_{j} \in \bigoplus_{j=1}^{r} \mathbb{C} \widehat{C}_{j}
$$

Now, notice that by definition the class sums $\widehat{C}_{j}(1 \leqslant j \leqslant r)$ are elements of the subring $\mathbb{Z} G$ of $\mathbb{C} G$, hence of the centre of $\mathbb{Z} G$.

\section{Corollary 16.3}

(a) $Z(\mathbb{Z} G)$ is finitely generated as a $\mathbb{Z}$-module.

(b) The centre $Z(\mathbb{Z} G)$ of the group ring $\mathbb{Z} G$ is integral over $\mathbb{Z}$; in particular the class sums $\hat{C}_{j}$ $(1 \leqslant j \leqslant r)$ are algebraic integers. 


\section{Proof:}

(a) It follows directly from the second part of the proof of Lemma 16.2 that the class sums $\widehat{C}_{j}(1 \leqslant j \leqslant r)$ span $Z(\mathbb{Z} G)$ as a $\mathbb{Z}$-module.

(b) The centre $Z(\mathbb{Z} G)$ is integral over $\mathbb{Z}$ by Theorem $D .2$ because it is finitely generated as a $\mathbb{Z}$-module by (a).

\section{Notation 16.4 (Central characters)}

If $\chi \in \operatorname{Irr}(G)$, then we may consider a $\mathbb{C}$-representation affording $\chi$, say $\rho^{\chi}: G \longrightarrow \mathrm{GL}\left(\mathbb{C}^{n(x)}\right)=$ Aut $_{\mathbb{C}}\left(\mathbb{C}^{n(\chi)}\right)$ with $n(\chi):=\chi(1)$. This group homomorphism extends by $\mathbb{C}$-linearity to a $\mathbb{C}$-algebra homomorphism

$$
\begin{aligned}
& \tilde{\rho}^{x}: \quad \mathbb{C} G \quad \longrightarrow \operatorname{End}_{\mathbb{C}}\left(\mathbb{C}^{n(x)}\right) \\
& a=\sum_{g \in G} \lambda_{g} g \quad \mapsto \quad \tilde{\rho}^{\chi}(a)=\sum_{g \in G} \lambda_{g} \rho^{\chi}(g) .
\end{aligned}
$$

Now, if $z \in Z(\mathbb{C} G)$, then for each $g \in G$, we have

$$
\widetilde{\rho}^{\chi}(z) \tilde{\rho}^{\chi}(g)=\widetilde{\rho}^{\chi}(z g)=\widetilde{\rho}^{\chi}(g z)=\widetilde{\rho}^{\chi}(g) \widetilde{\rho}^{\chi}(z) .
$$

As we have already seen in Chapter 2 on Schur's Lemma this means that $\widetilde{\rho}^{\chi}(z)$ is $\mathbb{C} G$-linear. This holds in particular if $z$ is a class sum. Therefore, by Schur's Lemma, for each $1 \leqslant j \leqslant r$ there exists a scalar $\omega_{\chi}\left(\widehat{C}_{j}\right) \in \mathbb{C}$ such that

$$
\tilde{\rho}^{\chi}\left(\widehat{C}_{j}\right)=\omega_{\chi}\left(\widehat{C}_{j}\right) \cdot I_{n(\chi)}
$$

The functions defined by

$$
\begin{aligned}
\omega_{\chi}: Z(\mathbb{C} G) & \longrightarrow \mathbb{C} \\
\widehat{C}_{j} & \mapsto \omega_{\chi}\left(\widehat{C}_{j}\right)
\end{aligned}
$$

and extended by $\mathbb{C}$-linearity to the whole of $Z(\mathbb{C} G)$, where $\chi$ runs through $\operatorname{Irr}(G)$, are called the central characters of $\mathbb{C} G$ (or simply of $G$ ).

\section{Remark 16.5}

If $z \in Z(G)$, then $[z]=\{z\}$ and therefore the corresponding class sum is $z$ itself. Therefore, we may see the functions $\left.\omega_{X}\right|_{Z(G)}: Z(G) \longrightarrow \mathbb{C}$ as representations of $Z(G)$ of degree 1 , or equivalently as linear characters of $Z(G)$.

\section{Theorem 16.6 (Integrality Theorem)}

The values $\omega_{\chi}\left(\widehat{C}_{j}\right)(\chi \in \operatorname{Irr}(G), 1 \leqslant j \leqslant r)$ of the central characters of $G$ are algebraic integers. Moreover,

$$
\omega_{\chi}\left(\widehat{C}_{j}\right)=\frac{\left|C_{j}\right|}{\chi(1)} \chi\left(g_{j}\right) \quad \forall \chi \in \operatorname{Irr}(G), \forall 1 \leqslant j \leqslant r .
$$

Proof: Let $\chi \in \operatorname{Irr}(G)$ and $1 \leqslant j \leqslant r$. By Corollary 16.3 the class sum $\widehat{C}_{j}$ is an algebraic integer. Thus there exist integers $n \in \mathbb{Z}_{>0}$ and $a_{0}, \ldots, a_{n-1} \in \mathbb{Z}$ such that $\widehat{C}_{j}^{n}+a_{n-1} \widehat{C}_{j}^{n-1}+\ldots+a_{0}=0$. Applying $\omega_{\chi}$ yields $\omega_{\chi}\left(\widehat{C}_{j}\right)^{n}+a_{n-1} \omega_{\chi}\left(\widehat{C}_{j}\right)^{n-1}+\ldots+a_{0}=\omega_{\chi}(0)=0$, so that $\omega_{\chi}\left(\widehat{C}_{j}\right)$ is also an algebraic integer. Now, according to Notation 16.4 we have

$$
\chi(1) \omega_{\chi}\left(\widehat{C}_{j}\right)=\operatorname{Tr}\left(\widetilde{\rho}^{\chi}\left(\widehat{C}_{j}\right)\right)=\operatorname{Tr}\left(\sum_{g \in C_{j}} \rho^{\chi}(g)\right)=\sum_{g \in C_{j}} \operatorname{Tr}\left(\rho^{\chi}(g)\right)=\sum_{g \in C_{j}} \chi(g)=\left|C_{j}\right| \chi(g),
$$


where the last equality holds because characters are class functions. The claim follows.

\section{Corollary 16.7}

If $\chi \in \operatorname{Irr}(G)$, then $\chi(1)$ divides $|G|$.

Proof: By the 1st Orthogonality Relations we have

$$
\frac{|G|}{\chi(1)}=\frac{|G|}{\chi(1)}\langle\chi, \chi\rangle_{G}=\frac{1}{\chi(1)} \sum_{g \in G} \chi(g) \chi\left(g^{-1}\right)=\frac{1}{\chi(1)} \sum_{j=1}^{r}\left|C_{j}\right| \chi\left(g_{j}\right) \chi\left(g_{j}^{-1}\right)=\sum_{j=1}^{\sum_{j}} \underbrace{\frac{\left|C_{j}\right|}{\chi(1)} \chi\left(g_{j}\right)}_{\omega_{\chi}\left(\hat{C}_{j}\right)} \chi\left(g_{j}^{-1}\right) .
$$

Now, for each $1 \leqslant j \leqslant r, \omega_{\chi}\left(g_{j}\right)$ is an algebraic integer by the Integrality Theorem and $\chi\left(g_{j}^{-1}\right)$ is an algebraic integer by Corollary 15.1. Hence $|G| / \chi(1)$ is an algebraic integer because these form a subring of $\mathbb{C}$. Moroever, clearly $|G| / \chi(1) \in \mathbb{Q}$. As the algebraic integers in $\mathbb{Q}$ are just the elements of $\mathbb{Z}$, we obtain that $|G| / \chi(1) \in \mathbb{Z}$, as claimed.

\section{Example 8 (The degrees of the irreducible characters of $\mathrm{GL}_{3}\left(\mathbb{F}_{2}\right)$ )}

The group $G:=\mathrm{GL}_{3}\left(\mathbb{F}_{2}\right)$ is a simple group of oder

$$
|G|=\# \mathbb{F}_{2} \text {-bases of } \mathbb{E}_{2}^{3}=\left(2^{3}-1\right)\left(2^{3}-2\right)\left(2^{3}-2^{2}\right)=168=2^{3} \cdot 3 \cdot 7 .
$$

For the purpose of this example we accept without proof that $G$ is simple and that it has 6 conjugacy classes.

Question: can we compute the degrees of the irreducible characters of $G_{1} L_{3}\left(\mathbb{F}_{2}\right)$ ?

(1) By the above $|\operatorname{Irr}(G)|=|C(G)|=6$ and the degree formula yields:

$$
1+\sum_{i=2}^{6} x_{i}(1)^{2}=|G|=168
$$

(2) Next, as $G$ is simple non-abelian, $G=G^{\prime}$ and therfeore $G$ has $\left|G: G^{\prime}\right|=1$ linear characters by Corollary 14.8, namely

$$
\chi_{i}(1) \geqslant 2 \text { for each } 2 \leqslant i \leqslant 6 .
$$

Thus, at this stage, we would have the following possibilities for the degrees of the 6 irreducible characters of $G$ :

\begin{tabular}{cccccc}
\hline$\chi_{1}(1)$ & $\chi_{2}(1)$ & $\chi_{3}(1)$ & $\chi_{4}(1)$ & $\chi_{5}(1)$ & $\chi_{6}(1)$ \\
\hline \hline 1 & 2 & 4 & 5 & 6 & 9 \\
1 & 2 & 3 & 3 & 8 & 9 \\
1 & 2 & 5 & 5 & 7 & 8 \\
1 & 2 & 4 & 7 & 7 & 7 \\
1 & 3 & 3 & 6 & 7 & 8 \\
\hline
\end{tabular}

(3) By Corollary 16.7 we now know that $\chi_{i}(1)|| G \mid$ for each $2 \leqslant i \leqslant 6$. Therefore, as $5 \nmid|G|$ and $9 \nmid|G|$, the first three rows can already be discarded: 


\begin{tabular}{cccccc}
\hline$\chi_{1}(1)$ & $\chi_{2}(1)$ & $\chi_{3}(1)$ & $\chi_{4}(1)$ & $\chi_{5}(1)$ & $\chi_{6}(1)$ \\
\hline 1 & 2 & 4 & 5 & 6 & 9 \\
1 & 2 & 3 & 3 & 8 & 9 \\
1 & 2 & 5 & 5 & 7 & 8 \\
1 & 2 & 4 & 7 & 7 & 7 \\
1 & 3 & 3 & 6 & 7 & 8 \\
\hline
\end{tabular}

(4) In order to eliminate the last-but-one possibility, we apply [Exercise 21(b), Sheet 6] saying that a simple group cannot have an irreducible character of degree 2 . Hence

$$
\chi_{1}(1)=1, \chi_{2}(1)=3, \chi_{3}(1)=3, \chi_{4}(1)=6, \chi_{5}(1)=7, \chi_{6}(1)=8
$$

\section{Exercise 16.8 (Exercise 22, Sheet 6)}

Let $G$ be a finite group of odd order and, as usual, let $r$ denote the number of conjugacy classes of $G$. Use character theory to prove that

$$
r \equiv|G| \quad(\bmod 16)
$$

[Hint: Label the set $\operatorname{Irr}(G)$ of irreducible characters taking dual characters into account. Use the divisibility property of Corollary 16.7]

\section{The Centre of a Character}

\section{Definition 17.1 (Centre of a character)}

The centre of a character $\chi$ of $G$ is $Z(\chi):=\{g \in G|| \chi(g) \mid=\chi(1)\}$.

Note: Recall that in contrast, $\chi(g)=\chi(1) \Leftrightarrow g \in \operatorname{ker}(\chi)$.

\section{Example 9}

Recall from Example 5 that the character table of $G=S_{3}$ is

\begin{tabular}{c|ccc} 
& Id & $(12)$ & $(123)$ \\
\cline { 2 - 4 }$\chi_{1}$ & 1 & 1 & 1 \\
$\chi_{2}$ & 1 & -1 & 1 \\
$\chi_{3}$ & 2 & 0 & -1
\end{tabular}

Hence $Z\left(\chi_{1}\right)=Z\left(\chi_{2}\right)=G$ and $Z\left(\chi_{3}\right)=\{\mathrm{Id}\}$.

\section{Lemma 17.2}

If $\rho: G \longrightarrow \mathrm{GL}(V)$ is a $\mathbb{C}$-representation with character $\chi$ and $g \in G$, then:

$$
|\chi(g)|=\chi(1) \Longleftrightarrow \rho(g) \in \mathbb{C}^{\times} \mid \mathrm{d}_{V} .
$$

In other words $Z(X)=\rho^{-1}\left(\mathbb{C}^{\times} \operatorname{Id} v\right)$. 
Proof: Let $n:=\chi(1)$. Recall that we can find a $\mathbb{C}$-basis $B$ of $V$ such that $(\rho(g))_{B}$ is a diagonal matrix with diagonal entries $\varepsilon_{1}, \ldots, \varepsilon_{n}$ which are $o(g)$-th roots of unity. Hence $\varepsilon_{1}, \ldots, \varepsilon_{n}$ are the eigenvalues of $\rho(g)$. Applying the Cauchy-Schwartz inequality to the vectors $v:=\left(\varepsilon_{1}, \ldots, \varepsilon_{n}\right)$ and $w:=(1, \ldots, 1)$ in $\mathbb{C}^{n}$ yields

$$
|\chi(g)|=\left|\varepsilon_{1}+\ldots+\varepsilon_{n}\right|=|\langle v, w\rangle| \leqslant\|v\| \cdot\|w\|=\sqrt{n} \sqrt{n}=n=\chi(1)
$$

and equality implies that $v$ and $w$ are $\mathbb{C}$-linearly dependent so that $\varepsilon_{1}=\ldots=\varepsilon_{n}=: \varepsilon$. Therefore $\rho(g) \in \mathbb{C}^{\times} \mathbb{I}_{V}$. Conversely, if $\rho(g) \in \mathbb{C}^{\times} \mathbb{I d}_{V}$, then there exists $\lambda \in \mathbb{C}^{\times}$such that $\rho(g)=\lambda \mathbb{I}_{V}$. Therefore the eigenvalues of $\rho(g)$ are all equal to $\lambda$, i.e. $\lambda=\varepsilon_{1}=\ldots=\varepsilon_{n}$ and therefore

$$
|\chi(g)|=|n \lambda|=n|\lambda|=n \cdot 1=n .
$$

\section{Proposition 17.3}

Let $\chi$ be a character of $G$. Then:

(a) $Z(\chi) \unlhd G$;

(b) $\operatorname{ker}(\chi) \unlhd Z(\chi)$ and $Z(\chi) / \operatorname{ker}(\chi)$ is a cyclic group;

(c) if $\chi$ is irreducible, then $Z(\chi) / \operatorname{ker}(\chi)=Z(G / \operatorname{ker}(\chi))$.

Proof: Let $\rho: G \longrightarrow \mathrm{GL}(V)$ be a $\mathbb{C}$-representation affording $\chi$ and set $n:=\chi(1)$.

(a) Clearly $\mathbb{C}^{\times} \operatorname{Id} V \leqslant Z(G L(V))$ and hence $\mathbb{C}^{\times} \operatorname{Id} v \unlhd G L(V)$. Therefore, by Lemma 17.2,

$$
Z(\chi)=\rho^{-1}\left(\mathbb{C}^{\times} \operatorname{Id} V\right) \unlhd G
$$

as the pre-image under a group homomorphism of a normal subgroup.

(b) By the definitions of the kernel and of the centre of a character, we have $\operatorname{ker}(x) \subseteq Z(\chi)$. Therefore $\operatorname{ker}(\chi) \unlhd Z(\chi)$ by (a). If $g \in Z(\chi)$, then by Lemma 17.2 restriction to $Z(\chi)$ yields a group homomorphism

$$
\left.\rho\right|_{Z(x)}: Z(X) \longrightarrow \mathbb{C}^{\times} \mid \mathrm{d}_{V}
$$

with $\operatorname{kernel} \operatorname{ker}(\chi)$. Therefore, by the 1st ismomorphism theorem, $Z(\chi) / \operatorname{ker}(\chi)$ is isomorphic to a finite subgroup of $\mathbb{C}^{\times} \mathbb{I d}_{V} \cong \mathbb{C}^{\times}$, hence is cyclic (C.f. e.g. EZT).

(c) By the arguments of (a) and (b) we have

$$
Z(\chi) / \operatorname{ker}(\chi) \cong \rho(Z(\chi)) \leqslant Z(\rho(G)) .
$$

Applying again the first isomorphism theorem we have $\rho(G) \cong G / \operatorname{ker}(\rho)$, hence

$$
Z(\rho(G)) \cong Z(G / \operatorname{ker}(\rho))=Z(G / \operatorname{ker}(\chi)) .
$$

Now let $\bar{g}=g \operatorname{ker}(\chi) \in Z(G / \operatorname{ker}(\chi))$. As $\chi$ is irreducible, $\rho(g)=\lambda \operatorname{ld}_{V}$ for some $\lambda \in \mathbb{C}^{\times}$by Schur's Lemma. Thus $g \in Z(X)$ and it follows that

$$
Z(G / \operatorname{ker}(\chi)) \leqslant Z(\chi) / \operatorname{ker}(\chi) .
$$

\section{Exercise 17.4 (Exercise 23, Sheet 6)}

Prove that if $\chi \in \operatorname{Irr}(G)$, then $Z(G) \leqslant Z(\chi)$ and deduce that $\bigcap_{\chi \in \operatorname{Irr}(G)} Z(\chi)=Z(G)$. 


\section{Remark 17.5 (See [Exercise 24, Sheet 6])}

If $\chi$ is an irreducible character of degree $n$ then $n$ divides $|G: Z(\chi)|$, and hence divides $|G: Z(G)|$.

This allows us to prove an important criterion, due to Burnside, for character values to be zero.

\section{Theorem 17.6 (Burnside)}

Let $\chi \in \operatorname{Irr}(G)$ and let $C=[g]$ be a conjugacy class of $G$ such that $\operatorname{gcd}(\chi(1),|C|)=1$. Then $\chi(g)=0$ or $g \in Z(\chi)$.

Proof: $\operatorname{As} \operatorname{gcd}(\chi(1),|C|)=1$, there exist $u, v \in \mathbb{Z}$ such that $u \chi(1)+v|C|=1$ Set $\alpha:=\frac{\chi(g)}{\chi(1)}$. Then

$$
\alpha=\frac{\chi(g)}{\chi(1)} \cdot 1=\frac{\chi(g)}{\chi(1)}(u \chi(1)+v|C|)=u \chi(g)+v \frac{|C| \chi(g)}{\chi(1)}=u \chi(g)+v \omega_{\chi}(C)
$$

is an algebraic integer because both $\chi(g)$ and $\omega_{\chi}(C)$ are. Now, set $m:=|\langle g\rangle|$ and let $\zeta_{m}:=e^{\frac{2 \pi i}{m}}$. As $\chi(g)$ is a sum of $m$-th roots of unity, certainly $\chi(g) \in \mathbb{Q}\left(\zeta_{m}\right)$. Let $\mathcal{G}$ be the Galois group of the Galois extension $\mathbb{Q} \subseteq \mathbb{Q}\left(\zeta_{m}\right)$. Then for each field automorphism $\sigma \in \mathcal{G}, \sigma(\alpha)$ is also an algebraic integer because $\alpha$ and $\sigma(\alpha)$ are roots of the same monic integral polynomial. Hence $\beta:=\prod_{\sigma \in \mathcal{G}} \sigma(\alpha)$ is also an algebaric integer and because $\sigma(\beta)=\beta$ for every $\sigma \in \mathcal{G}, \beta$ is an element of the fixed field of $\mathcal{G}$, namely $\beta \in \mathbb{Q}$ (Galois theory). Therefore $\beta \in \mathbb{Z}$.

If $g \in Z(\chi)$, then there is nothing to do. Thus we may assume that $g \notin Z(\chi)$. Then $|\chi(g)| \neq \chi(1)$, so that by Property 7.4(c) we must have $|\chi(g)|<\chi(1)$ and hence $|\alpha|<1$. Now, again by Property 7.4(b), $\chi(g)=\varepsilon_{1}+\ldots+\varepsilon_{n}$ with $n=\chi(1)$ and $\varepsilon_{1}, \ldots, \varepsilon_{n} m$-th roots of unity. Therefore, for each $\sigma \in \mathcal{G} \backslash\{\mathrm{Id}\}$, we have $\sigma(\chi(g))=\sigma\left(\varepsilon_{1}\right)+\ldots+\sigma\left(\varepsilon_{n}\right)$ with $\sigma\left(\varepsilon_{1}\right), \ldots, \sigma\left(\varepsilon_{n}\right) m$-th roots of unity, because $\varepsilon_{1}, \ldots, \varepsilon_{n}$ are. It follows that

$$
|\sigma(\chi(g))|=\left|\sigma\left(\varepsilon_{1}\right)+\ldots+\sigma\left(\varepsilon_{n}\right)\right| \leqslant\left|\sigma\left(\varepsilon_{1}\right)\right|+\ldots+\left|\sigma\left(\varepsilon_{n}\right)\right|=n=\chi(1)
$$

and hence

$$
|\sigma(\alpha)|=\frac{1}{\chi(1)}|\sigma(\chi(g))| \leqslant \frac{\chi(1)}{\chi(1)}=1 .
$$

Thus

$$
|\beta|=\left|\prod_{\sigma \in \mathcal{G}} \sigma(\alpha)\right|=\underbrace{|\alpha|}_{<1} \cdot \prod_{\sigma \in \mathcal{G} \backslash\{\mid \mathrm{d}\}} \underbrace{|\sigma(\alpha)|}_{\leqslant 1}<1 .
$$

The only way an integer satisfies this inequality is $\beta=0$. Thus $\alpha=0$ as well, which implies that $\chi(g)=0$.

\section{Corollary 17.7}

Assume now that $G$ is a non-abelian simple group. In the situation of Theorem 17.6 if we assume moreover that $\chi(1)>1$ and $C \neq\{1\}$, then it is always the case that $\chi(g)=0$.

Proof: We see that then either $\chi(g)=0$ or $Z(\chi)$ is a non-trivial proper normal subgroup of $G$. Indeed, if $\chi(g) \neq 0$, then Theorem 17.6 implies that $g \in Z(\chi)$, so $Z(\chi) \neq 1$. Now, as $G$ is non-abelian simple we have $Z(\chi)=G$. On the other hand, the fact that $G$ is simple also tells us that $\operatorname{ker}(\chi)=1$ (if it were $G$, then $\chi$ would be reducible). Then it follows from Proposition 17.3 that

$$
G=Z(\chi) / \operatorname{ker}(\chi)=Z(G / \operatorname{ker}(\chi))=Z(G)=1 .
$$

A contradiction. 


\section{Burnside's $p^{a} q^{b}$-Theorem}

Character theory has many possible applications to the to the structure of finite groups. We consider in this section on of the most famous of these: the proof of Burnside's $p^{a} q^{b}$ theorem.

\section{Example 10}

To begin with we consider two possible minor applications of character theory to finite groups. Both are results of the Einfürung in die Algebra, for which you have already seen purely group-theoretic proofs.

(a) $G$ finite group such that $|G|=p^{2}$ for some prime number $p \Longrightarrow G$ is abelian.

- Proof using character theory. By Corollary 16.7 we have $\chi(1)|| G \mid$ for each $\chi \in \operatorname{Irr}(G)$. Thus

$$
\chi(1) \in\left\{1, p, p^{2}\right\} .
$$

Therefore the class equation reads

$$
p^{2}=|G|=\sum_{\chi \in \operatorname{lrr}(G)} \chi(1)^{2}=\underbrace{1_{G}(1)^{2}}_{=1}+\sum_{\substack{x \in \operatorname{lrr}(G) \\ \chi \neq 1_{G}}} \chi(1)^{2},
$$

which implies that it is not possible that the degree of an irreducible character of $G$ is $p$ or $p^{2}$. In other words, all the irreducible characters of $G$ are linear, and thus $G$ is abelian by Corollary 14.8.

(b) $G$ is a non-trivial $p$-group $\Longrightarrow G$ is soluble.

[Recall from the Einfürung in die Algebra that a finite group $G$ is soluble if it admits a chain of subgroups

$$
1=G_{0}<G_{1}<\ldots<G_{s}=G
$$

such that for $1 \leqslant i \leqslant s, G_{i-1} \triangleleft G_{i}$ and $G_{i} / G_{i-1}$ is cyclic of prime order. Moreover, we have the following very useful solubility criterion, sometimes coined "the sandwich principle": if $H \unlhd G$ is a normal subgroup, then the group $G$ is soluble if and only if both $G$ and $G / H$ are soluble.]

- Proof using character theory. By induction on $|G|=: p^{a}\left(a \in \mathbb{Z}_{>0}\right)$. If $|G|=p$ or $|G|=p^{2}$, then $G$ is abelian (cyclic in the former case). Finite abelian groups are clearly soluble because they are products of cyclic groups of prime power order.

Therefore, we may assume that $|G| \geqslant p^{3}$. As in (a) Corollary 16.7 implies that

$$
\chi(1) \in\left\{1, p, p^{2}, \ldots, p^{a}\right\} \quad \text { for each } \chi \in \operatorname{Irr}(G) .
$$

Now, again the degree formula yields

$$
p^{a}=|G|=1+\sum_{\substack{x \in \operatorname{lrr}(G) \\ \chi \neq 1_{G}}} \chi(1)^{2} .
$$

and for this equality to hold, there must be at least $p$ linear characters of $G$ (including the trivial character). Thus it follows from Corollary 14.8 that $G^{\prime} \lessgtr G$. Hence both $G^{\prime}$ and $G / G^{\prime}$ are soluble by the induction hypothesis $\Rightarrow G$ is soluble by the sandwich principle. 


\section{Theorem 18.1 (Burnside)}

Let $G$ be a finite non-abelian simple group. If $C$ is a conjugacy class of $G$ such that $|C|=p^{a}$ with $p$ prime and $a \in \mathbb{Z}_{\geqslant 0}$, then $C=\{1\}$.

Proof: Assume ab absurdo that $C \neq\{1\}$ and choose $g \in C$. In particular $g \neq 1$. Since $G$ is non-abelian simple $G=G^{\prime}$ and it follows from Corollary 14.8 that the unique linear character of $G$ is the trivial character. Hence for each $\chi \in \operatorname{Irr}(G) \backslash\left\{1_{G}\right\}$ we have either $p \mid \chi(1) \operatorname{or} \operatorname{gcd}(\chi(1),|C|)=1$. Thus $\chi(g)=0$ if $p \nmid \chi(1)$ and $\chi \neq 1_{G}$ by Corollary 17.7. Therefore the Second Orthogonality Relations read

$$
0=1+\sum_{\substack{\chi \in \operatorname{lrr}(G) \\ \chi \neq 1 G}} \underbrace{\chi(g)}_{\substack{=0 \text { if } \\ p \nmid \chi(1)}} \underbrace{\overline{\chi(1)}}_{=\chi(1)}=1+\sum_{\substack{\chi \in \operatorname{lr}(G) \\ p \mid \chi(1)}} \chi(g) \chi(1)
$$

and dividing by $p$ yields

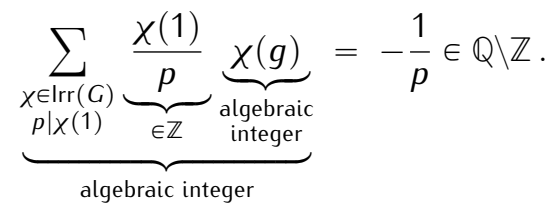

This contradicts the fact that rational numbers which are algebraic integers are integers. It follows that $g=1$ is the only possibility and hence $C=\{1\}$.

As a consequence, we obtain Burnside's $p^{a} q^{b}$ theorem, which can be found in the literature under two different forms. The first version provides us with a "non-simplicity" criterion and the second version with a solubility criterion, which is extremely hard to prove by purely group theoretic methods.

\section{Theorem 18.2 (Burnside's $p^{a} q^{b}$ Theorem, "simple" version)}

Let $p, q$ be prime numbers and let $a, b \in \mathbb{Z}_{\geqslant 0}$ be integers such that $a+b \geqslant 2$. If $G$ is a finite group of order $p^{a} q^{b}$, then $G$ is not simple.

Proof: First assume that $a=0$ or $b=0$. Then $G$ is a $q$-group with $q^{2}|| G \mid$, resp. a p-group with $p^{2}|| G \mid$. Therefore the centre of $G$ is non-trivial (Einfürung in die Algebra), thus of non-trivial prime power order. Therefore there exists an element $g \in Z(G)$ of order $q$ (resp. $p$ ) and $1 \neq\langle g\rangle \triangleleft G$ is a proper non-trivial normal subgroup. Hence $G$ is not simple.

We may now assume that $a \neq 0 \neq b$. Let $Q \in \operatorname{Syl}_{q}(G)$ be a Sylow $q$-subgroup of $G$ (i.e. $|Q|=q^{b}$ ). Again, as $Q$ is a $q$-group, we have $Z(Q) \neq\{1\}$ and we can choose $g \in Z(Q) \backslash\{1\}$. Then

$$
Q \leqslant C_{G}(g)
$$

and therefore the Orbit-Stabiliser Theorem yields

$$
|[g]|=\left|G: C_{G}(g)\right|=p^{r}
$$

for some non-negative integer $r \leqslant a$. If $r=0$, then $p^{r}=1$ and $G=C_{G}(g)$, so that $g \in Z(G)$. Hence $Z(G) \neq\{1\}$ and $G$ is not simple by the same argument as above. If $p^{r}>1$, then $G$ cannot be simple by Theorem 18.1 .

\section{Theorem 18.3 (Burnside's $p^{a} q^{b}$ Theorem, "soluble" version)}

Let $p, q$ be prime numbers and $a, b \in \mathbb{Z}_{\geqslant 0}$. Then any finite group of order $p^{a} q^{b}$ is soluble.

Proof: Let $G$ be a finite group of order $p^{a} q^{b}$. We proceed by induction on $a+b$.

- $a+b \in\{0,1\} \Longrightarrow G$ is either trivial or cyclic of prime order, hence clearly soluble. 
- $a+b \geqslant 2 \Longrightarrow G$ is not simple by the "simple" version of Burnside's $p^{a} q^{b}$ theorem. Hence there exists a proper non-trivial normal subgroup $H$ in $G$ and both $|H|,|G / H|<p^{a} q^{b}$. Therefore both $H$ and $G / H$ are soluble by the induction hypothesis. Thus $G$ is soluble by the sandwich principle. 
In this chapter we present important methods to construct / relate characters of a group, given characters of subgroups or overgroups. The main idea is that we would like to be able to use the character tables of groups we know already in order to compute the character tables of subgroups or overgroups of these groups.

Notation: throughout this chapter, unless otherwise specified, we let:

- $G$ denote a finite group, $H \leqslant G$ and $N \unlhd G$.

$\cdot K:=\mathbb{C}$ be the field of complex numbers;

- $\operatorname{Ir}(G):=\left\{\chi_{1}, \ldots, \chi_{r}\right\}$ denote the set of pairwise distinct irreducible characters of $G$;

- $C_{1}=\left[g_{1}\right], \ldots, C_{r}=\left[g_{r}\right]$ denote the conjugacy classes of $G$, where $g_{1}, \ldots, g_{r}$ is a fixed set of representatives; and

. we use the convention that $\chi_{1}=1 G$ and $g_{1}=1 \in G$.

In general, unless otherwise stated, all groups considered are assumed to be finite and all $\mathbb{C}$-vector spaces / modules over the group algebra considered are assumed to be finite-dimensional.

\section{Induction and Restriction}

We aim at inducing and restricting characters from subgroups, resp. overgroups. We start with the operation of induction, which is a subtle operation to construct a class function on $G$ from a given class function on a subgroup $H \leqslant G$. We will focus on characters in a second step.

\section{Definition 19.1 (Induced class function)}

Let $H \leqslant G$ and $\varphi \in \mathcal{C l}(H)$ be a class function on $H$. Then the induction of $\varphi$ from $H$ to $G$ is

$$
\begin{aligned}
\operatorname{lnd}_{H}^{G}(\varphi)=: \varphi \uparrow_{H}^{G}: \quad G & \longrightarrow \mathbb{C} \\
\varphi & \mapsto \varphi \uparrow_{H}^{G}:=\frac{1}{|H|} \sum_{x \in G} \varphi^{\circ}\left(x^{-1} g x\right),
\end{aligned}
$$

where for $y \in G, \varphi^{\circ}(y):= \begin{cases}\varphi(y) & \text { if } y \in H \\ 0 & \text { if } y \notin H\end{cases}$ 


\section{Remark 19.2}

With the notation of Definition 19.1 the function $\varphi \uparrow_{H}^{G}$ is again a class function, because for every $g, y \in G$,

$$
\varphi \uparrow_{H}^{G}\left(y^{-1} g y\right)=\frac{1}{|H|} \sum_{x \in G} \varphi^{\circ}\left(x^{-1} y^{-1} g y x\right) \stackrel{s:=y x}{=} \frac{1}{|H|} \sum_{s \in G} \varphi^{\circ}\left(s^{-1} g s\right)=\varphi \uparrow_{H}^{G}(g) .
$$

Moreover, the map $\operatorname{Ind}_{H}^{G}: \mathcal{C l}(H) \longrightarrow \mathcal{C l}(G), \varphi \mapsto \varphi \uparrow_{H}^{G}$ is $\mathbb{C}$-linear [Exercise 25(d), Sheet 7].

In contrast, the operation of restriction is based on the more elementary idea that any map can be restricted to a subset of its domain. For class functions / representations / characters we are essentially interested in restricting these (seen as maps) to subgroups.

\section{Definition 19.3 (Restricted class function)}

Let $H \leqslant G$ and $\psi \in \mathcal{C l}(G)$ be a class function on $G$. Then the restriction of $\psi$ from $G$ to $H$ is

$$
\operatorname{Res}_{H}^{G}(\psi)=\psi \downarrow_{H}^{G}=\left.\psi\right|_{H} .
$$

This is obviously again a class function on $H$.

\section{Remark 19.4}

If $\psi$ is a character of $G$ afforded by the $\mathbb{C}$-representation $\rho: G \longrightarrow G L(V)$, then clearly $\psi \downarrow_{H}^{G}$ is the character afforded by the $\mathbb{C}$-representation $\left.\rho\right|_{H}: H \longrightarrow \mathrm{GL}(V)$.

\section{Exercise 19.5 (Exercise 25, Sheet 7)}

Let $H \leqslant J \leqslant G$. Prove that:

(a) $\varphi \in \mathcal{C l}(H) \Longrightarrow\left(\varphi \uparrow_{H}^{\jmath}\right) \uparrow_{j}^{G}=\varphi \uparrow_{H}^{G}$ (transitivity of induction);

(b) $\psi \in \mathcal{C l}(G) \Longrightarrow\left(\psi \downarrow_{j}^{G}\right) \downarrow_{H}^{J}=\psi \downarrow_{H}^{G}$ (transitivity of restriction);

(c) $\varphi \in \mathcal{C l}(H)$ and $\psi \in \mathcal{C l}(G) \Longrightarrow \psi \cdot \varphi \uparrow_{H}^{G}=\left(\psi \downarrow_{H}^{G} \cdot \varphi\right) \uparrow_{H}^{G} \quad$ (Frobenius formula).

\section{Theorem 19.6 (Frobenius reciprocity)}

Let $H \leqslant G$, let $\varphi \in \mathcal{C l}(H)$ be a class function on $H$, and let $\psi \in \mathcal{C l}(G)$ be a class function on $G$. Then

$$
\left\langle\varphi \uparrow_{H}^{G}, \psi\right\rangle_{G}=\left\langle\varphi, \psi \downarrow_{H}^{G}\right\rangle_{H}
$$

Proof: By the definitions of the scalar product and of the induction a direct computation yields:

$$
\begin{aligned}
\left\langle\varphi \uparrow_{H}^{G}, \psi\right\rangle_{G}=\frac{1}{|G|} \sum_{g \in G} \varphi \uparrow_{H}^{G}(g) \psi\left(g^{-1}\right) & =\frac{1}{|G|} \sum_{g \in G} \frac{1}{|H|} \sum_{x \in G} \varphi^{\circ}\left(x^{-1} g x\right) \psi\left(g^{-1}\right) \\
& =\frac{1}{|G||H|} \sum_{s \in G} \sum_{x \in G} \varphi^{\circ}(s) \psi\left(x s^{-1} x^{-1}\right) \\
& =\frac{1}{|H|} \sum_{s \in G} \varphi^{\circ}(s) \psi\left(s^{-1}\right)=\frac{1}{|H|} \sum_{s \in H} \varphi\left(s^{-1}\right) \psi(s)=\left\langle\varphi, \downarrow_{H}^{G}\right\rangle_{H},
\end{aligned}
$$

where for the third equality we set $s:=x^{-1} g x$ and the fourth equality comes from the fact that $\psi$ is a class function on $G$. 


\section{Corollary 19.7}

Let $H \leqslant G$ and let $\chi$ be a character of $H$ of degree $n$. Then the induced class function $\chi \uparrow_{H}^{G}$ is a character of $G$ of degree $n|G: H|$.

Proof: For $\psi \in \operatorname{Irr}(G)$ by Frobenius reciprocity we can set

$$
m_{\psi}:=\left\langle\chi \uparrow_{H}^{G}, \psi\right\rangle_{G}=\left\langle\chi, \psi \downarrow_{H}^{G}\right\rangle_{H} \in \mathbb{Z}_{\geqslant 0},
$$

which is an integer because both $\chi$ and $\psi \downarrow_{H}^{G}$ are characters of $H$. Therefore,

$$
\chi \uparrow \uparrow_{H}^{G}=\sum_{\psi \in \operatorname{Irr}(G)} m_{\psi} \psi
$$

is a non-negative integral linear combination of irreducible characters of $G$, hence a character of $G$. Moreover,

$$
\chi \uparrow_{H}^{G}(1)=\frac{1}{|H|} \sum_{\chi \in G} \chi^{\circ}(1)=\frac{1}{|H|}|G| \chi(1)=\chi(1)|G: H| .
$$

\section{Example 11}

(a) The restriction of the trivial character of $G$ from $G$ to $H$ is obviously the trivial character of $H$.

(b) If $H=\{1\}$, then $1_{\{1\}} \uparrow_{\{1\}}^{G}=\chi_{\text {reg }}$. Indeed, if $g \in G$ then

$$
1_{\{1\}} \uparrow_{\{1\}}^{G}(g)=\frac{1}{|\{1\}|} \sum_{x \in G} \underbrace{1_{\{1\}}^{\circ}\left(x^{-1} g x\right)}_{=0 \text { unless } g=1}=\delta_{1 g}|G|=\chi_{\text {reg }}(g)
$$

by Corollary 10.2.

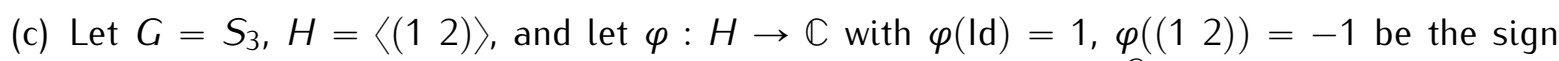
homomorphism on $H$. By the remark, it is enough to compute $\varphi \uparrow_{H}^{G}$ on representatives of the conjugacy classes of $S_{3}$, e.g. Id, (1 12$)$ and (1 $\left.\begin{array}{llll}1 & 2 & 3\end{array}\right)$ :

$$
\begin{gathered}
\varphi \uparrow_{H}^{G}(\text { Id })=\frac{1}{2} \sum_{x \in S_{3}} \varphi^{\circ}(\mathrm{Id})=3, \\
\varphi \uparrow_{H}^{G}\left(\left(\begin{array}{lll}
1 & 2 & 3
\end{array}\right)\right)=\frac{1}{2} \sum_{x \in S_{3}} \varphi^{\circ}\left(x^{-1}\left(\begin{array}{lll}
1 & 2 & 3
\end{array}\right) x\right)=0, \\
\varphi \uparrow_{H}^{G}\left(\left(\begin{array}{ll}
1 & 2
\end{array}\right)\right)=\frac{1}{2} \sum_{x \in S_{3}} \varphi^{\circ}\left(x^{-1}\left(\begin{array}{lll}
1 & 2
\end{array}\right) x\right)=\varphi^{\circ}\left(\left(\begin{array}{ll}
1 & 2
\end{array}\right)\right)+\varphi^{\circ}\left(\left(\begin{array}{lll}
1 & 3
\end{array}\right)\right)+\varphi^{\circ}\left(\left(\begin{array}{ll}
2 & 3
\end{array}\right)\right)=-1+0+0=-1 .
\end{gathered}
$$

Moreover we see from the character table of $S_{3}$ (Example 5) that $\varphi \uparrow_{H}^{G}=\chi_{2}+\chi_{3}$. But we can also compute with Frobenius reciprocity, that

$$
0=\left\langle\varphi, \chi_{1} \downarrow_{H}^{G}\right\rangle_{H}=\left\langle\varphi \uparrow_{H}^{G}, \chi_{1}\right\rangle_{G}
$$

and similarly

$$
1=\left\langle\varphi, \chi_{2} \downarrow_{H}^{G}\right\rangle_{H}=\left\langle\varphi \uparrow_{H}^{G}, \chi_{2}\right\rangle_{G} \quad \text { and } \quad 1=\left\langle\varphi, \chi_{3} \downarrow_{H}^{G}\right\rangle_{H}=\left\langle\varphi \uparrow_{H}^{G}, \chi_{3}\right\rangle_{G} .
$$




\section{Example 12 (The character table of the alternating group $A_{5}$ )}

The conjugacy classes of $G=A_{5}$ are

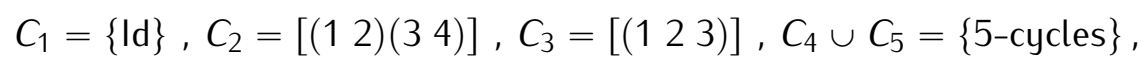

i.e. $g_{1}=\mathrm{Id}, g_{2}=\left(\begin{array}{ll}1 & 2\end{array}\right)\left(\begin{array}{l}3 \\ 4\end{array}\right), g_{3}=\left(\begin{array}{lll}1 & 2 & 3\end{array}\right)$ and $g \in C_{4} \Rightarrow o(g)=5$ and $g^{-1} \in C_{4}$ but $g^{2}, g^{3} \in C_{5}$ so that we can choose $g_{4}:=\left(\begin{array}{lllll}1 & 2 & 3 & 4 & 5\end{array}\right)$ and $g_{5}:=\left(\begin{array}{lllll}1 & 3 & 5 & 2 & 4\end{array}\right)$. This yields:

$$
\left|\operatorname{lr}\left(A_{5}\right)\right|=5 \text { and }\left|C_{1}\right|=1,\left|C_{2}\right|=15,\left|C_{3}\right|=20,\left|C_{4}\right|=\left|C_{5}\right|=12 .
$$

We obtain the character table of $A_{5}$ as follows:

- We know that the trivial character $1_{G}=\chi_{1}$ is one of the irreducible characters, hence we need to determine $\operatorname{Irr}\left(A_{5}\right) \backslash\left\{1_{G}\right\}=\left\{\chi_{2}, \chi_{3}, \chi_{4}, \chi_{5}\right\}$.

- Now, $H:=A_{4} \leqslant A_{5}$ and we have already computed the character table of $A_{4}$ in Exercise 14.10 (cf. solution of Exercise 19, Sheet 5). Therefore, inducing the trivial character of $A_{4}$ from $A_{4}$ to $A_{5}$ we obtain that

$$
\begin{aligned}
& 1_{H \uparrow}{ }_{H}^{G}(\mathrm{Id})=1 \cdot|G: H|=5 \quad \text { (see Cor. 19.7) } \\
& 1_{H \uparrow} \uparrow_{H}^{G}\left(\left(\begin{array}{ll}
1 & 2
\end{array}\right)(34)\right)=\frac{1}{12} \cdot 12=1 \\
& 1_{H} \uparrow_{H}^{G}\left(\left(\begin{array}{lll}
1 & 2 & 3
\end{array}\right)\right)=\frac{1}{12} \cdot 24=2 \\
& 1_{H} \uparrow_{H}^{G}(5 \text {-cycle })=\frac{1}{12} \cdot 0=0
\end{aligned}
$$

Now by Frobenius reciprocity

$$
\left\langle 1_{H \uparrow}^{G}, \chi_{1}\right\rangle_{G}=\langle 1_{H}, \underbrace{\chi_{1} \downarrow_{H}^{G}}_{=1_{H}}\rangle_{H}=1 .
$$

It follows (check it) that $\left\langle\mathbf{1}_{H} \uparrow_{H}^{G}-\chi_{1}, 1_{H} \uparrow_{H}^{G}-\chi_{1}\right\rangle_{G}=1$, so $1_{H} \uparrow_{H}^{G}-\chi_{1}$ is an irreducible character, say $\chi_{4}:=1_{H \uparrow}{ }_{H}^{G}-\chi_{1}$. The values of $\chi_{4}$ are given by $(4,0,1,-1,-1)$ on $C_{1}, C_{2}, C_{3}, C_{4}, C_{5}$ respectively.

- Next, as $A_{5}$ is a nonabelian simple group $A_{5} /\left[A_{5}, A_{5}\right]=1$, hence the trivial character is the unique linear character of $A_{5}$ and $\chi_{2}(1), \chi_{3}(1), \chi_{5}(1) \geqslant 3$. (You have also proved in Exercise 20 , Sheet 5 that simple groups do not have irreducible characters of degree 2.) Then the degree formula yields

$$
\chi_{2}(1)^{2}+\chi_{3}(1)^{2}+\chi_{5}(1)^{2}=\left|A_{5}\right|-\chi_{1}(1)^{2}-\chi_{4}(1)^{2}=20-1-16=43 .
$$

As degrees of characters must divide the group order, it follows from this formula that $\chi_{2}(1), \chi_{3}(1), \chi_{5}(1) \in\{3,4,5,6\}$, but then also that it is not possible to have an irreducible character of degree 6 . From this we easily see that only possibility, up to relabelling, is $\chi_{2}(1)=\chi_{3}(1)=3$ and $\chi_{5}(1)=5$. Hence at this stage, we already have the following part of the character table:

\begin{tabular}{c|ccccc} 
& $C_{1}$ & $C_{2}$ & $C_{3}$ & $C_{4}$ & $C_{5}$ \\
\cline { 2 - 6 }$\left|C_{k}\right|$ & 1 & 15 & 20 & 12 & 12 \\
$\left|C_{G}\left(g_{k}\right)\right|$ & 60 & 4 & 3 & 5 & 5 \\
\hline$\chi_{1}$ & 1 & 1 & 1 & 1 & 1 \\
$\chi_{2}$ & 3 &. &. &. &. \\
$\chi_{3}$ & 3 &. &. &. &. \\
$\chi_{4}$ & 4 & 0 & 1 & -1 & -1 \\
$\chi_{5}$ & 5 &. &. &. &.
\end{tabular}


- Next, we have that

$$
\begin{aligned}
\operatorname{gcd}\left(\chi_{2}(1),\left|C_{3}\right|\right)=\operatorname{gcd}\left(\chi_{3}(1),\left|C_{3}\right|\right) & =\operatorname{gcd}\left(\chi_{5}(1),\left|C_{4}\right|\right) \\
& =\operatorname{gcd}\left(\chi_{5}(1),\left|C_{4}\right|\right)=\operatorname{gcd}\left(\chi_{5}(1),\left|C_{5}\right|\right)=1,
\end{aligned}
$$

so that the corresponding character values must all be zero by Corollary 17.7 and we get:

\begin{tabular}{c|ccccc} 
& $C_{1}$ & $C_{2}$ & $C_{3}$ & $C_{4}$ & $C_{5}$ \\
\cline { 2 - 6 }$\left|C_{k}\right|$ & 1 & 15 & 20 & 12 & 12 \\
$\left|C_{G}\left(g_{k}\right)\right|$ & 60 & 4 & 3 & 5 & 5 \\
\hline$\chi_{1}$ & 1 & 1 & 1 & 1 & 1 \\
$\chi_{2}$ & 3 &. & 0 &. &. \\
$\chi_{3}$ & 3 &. & 0 &. &. \\
$\chi_{4}$ & 4 & 0 & 1 & -1 & -1 \\
$\chi_{5}$ & 5 &. &. & 0 & 0
\end{tabular}

- Applying the Orthogonality Relations yields:

1st, 3rd column $\Rightarrow \chi_{5}\left(g_{3}\right)=-1$ and the scalar product $\left\langle\chi_{1}, \chi_{5}\right\rangle_{G}=0 \Rightarrow \chi_{5}\left(g_{2}\right)=1$.

- Finally, to fill out the remaining gaps, we can induce from the cyclic subgroup $Z_{5}:=$ $\left\langle\left(\begin{array}{llll}1 & 2 & 3 & 4\end{array}\right)\right\rangle \leqslant A_{5}$ : choosing the non-trivial irreducible character $\psi$ of $Z_{5}$ which was denoted " $\chi_{3}$ " in Example 4 gives

$$
\psi \uparrow \bar{Z}_{5}=\left(12,0,0, \zeta^{2}+\zeta^{3}, \zeta+\zeta^{4}\right)
$$

where $\zeta=\exp (2 \pi i / 5)$ is a primitive 5 -th root of unity. Then we compute that

$$
\left\langle\psi \uparrow G_{Z_{5}}, \chi_{4}\right\rangle_{G}=1=\left\langle\psi \uparrow G_{Z_{5}}, \chi_{5}\right\rangle_{G} \quad \Longrightarrow \quad \psi \uparrow \bar{Z}_{5}-\chi_{4}-\chi_{5}=\left(3,-1,0,-\zeta-\zeta^{4},-\zeta^{2}-\zeta^{3}\right)
$$

and this character must be irreducible, because it is not the sum of 3 copies of the trivial character. Hence we set $\chi_{2}:=\psi \uparrow \bar{Z}_{5}-\chi_{4}-\chi_{5}$ and the values of $\chi_{3}$ then easily follow from the 2nd Othogonality Relations:

\begin{tabular}{c|ccccc} 
& $C_{1}$ & $C_{2}$ & $C_{3}$ & $C_{4}$ & $C_{5}$ \\
\cline { 2 - 6 }$\left|C_{k}\right|$ & 1 & 15 & 20 & 12 & 12 \\
$\left|C_{G}\left(g_{k}\right)\right|$ & 60 & 4 & 3 & 5 & 5 \\
\hline$\chi_{1}$ & 1 & 1 & 1 & 1 & 1 \\
$\chi_{2}$ & 3 & -1 & 0 & $-\zeta-\zeta^{4}$ & $-\zeta^{2}-\zeta^{3}$ \\
$\chi_{3}$ & 3 & -1 & 0 & $-\zeta^{2}-\zeta^{3}$ & $-\zeta-\zeta^{4}$ \\
$\chi_{4}$ & 4 & 0 & 1 & -1 & -1 \\
$\chi_{5}$ & 5 & 1 & -1 & 0 & 0
\end{tabular}

\section{Remark 19.8 (Induction of $\mathrm{KH}$-modules)}

If you have attended the lecture Commutative Algebra you have studied the tensor product of modules. In the M.Sc. lecture Representation Theory you will see that induction of modules is defined through a tensor product, extending the scalars from $\mathbb{C H}$ to $\mathbb{C} G$. More precisely, if $M$ is a $K H$-module, then the induction of $M$ from $H$ to $G$ is defined to be $K G \otimes_{K H} M$. Moreover, if $M$ affords the character $\chi$, then $K G \otimes_{K H} M$ affords the character $\chi \uparrow_{H}^{G}$. 


\section{Clifford Theory}

Clifford theory is a generic term for a series of results relating the representation / character theory of a given group $G$ to that of a normal subgroup $N \unlhd G$ through induction and restriction.

\section{Definition 20.1 (Conjugate class function / inertia group)}

Let $H \leqslant G$, let $\varphi \in \mathcal{C l}(H)$ and let $g \in G$.

(a) We define ${ }^{g} \varphi \in \mathcal{C l}\left(\mathrm{gHg}^{-1}\right)$ to be the class function on $\mathrm{gHg}^{-1}$ defined by

$$
{ }^{g} \varphi: g H g^{-1} \longrightarrow \mathbb{C}, x \mapsto \varphi\left(g^{-1} x g\right) .
$$

(b) The subgroup $\mathcal{I}_{G}(\varphi):=\left\{g \in G \mid{ }^{g} \varphi=\varphi\right\} \leqslant G$ is called the inertia group of $\varphi$ in $G$.

\section{Exercise 20.2 (Exercise 26, Sheet 7)}

With the notation of Definition 20.1, prove that:

(a) ${ }^{g} \varphi$ is indeed a class function on $\mathrm{gHg}^{-1}$;

(b) $\mathcal{I}_{G}(\varphi) \leqslant G$ and $H \leqslant \mathcal{I}_{G}(\varphi) \leqslant N_{G}(H)$;

(c) for $g, h \in G$ we have ${ }^{g} \varphi={ }^{h} \varphi \Leftrightarrow h^{-1} g \in \mathcal{I}_{G}(\varphi) \Leftrightarrow g \mathcal{I}_{G}(\varphi)=h \mathcal{I}_{G}(\varphi)$;

(d) if $\rho: H \longrightarrow \mathrm{GL}(V)$ is a $\mathbb{C}$-representation of $H$ with character $\chi$, then

$$
{ }^{g} \rho: g H^{-1} \longrightarrow \mathrm{GL}(V), x \mapsto \rho\left(g^{-1} x g\right)
$$

is $\mathbb{C}$-representation of $g \mathrm{Hg}^{-1}$ with character ${ }^{g} \chi$ and ${ }^{g} \chi(1)=\chi(1)$;

(e) if $J \leqslant H$ then ${ }^{g}\left(\varphi \downarrow_{\jmath}^{H}\right)=\left({ }^{g} \varphi\right) \downarrow_{g J g^{-1}}^{g H g^{-1}}$.

\section{Lemma 20.3}

(a) If $H \leqslant G, \varphi, \psi \in \mathcal{C l}(H)$ and $g \in G$, then $\left\langle{ }^{g} \varphi,{ }^{g} \psi\right\rangle_{g H g^{-1}}=\langle\varphi, \psi\rangle_{H}$.

(b) If $N \unlhd G$ and $g \in G$, then we have $\psi \in \operatorname{Irr}(N) \Leftrightarrow{ }^{g} \psi \in \operatorname{Irr}(N)$.

(c) If $N \unlhd G$ and $\psi$ is a character of $N$, then $\left(\psi \uparrow_{N}^{G}\right) \downarrow_{N}^{G}=\left|\mathcal{I}_{G}(\psi): N\right| \sum_{g \in\left[G / \mathcal{I}_{G}(\psi)\right]}{ }^{g} \psi$.

Proof: (a) Clearly

$$
\begin{aligned}
&\left\langle{ }^{g} \varphi,{ }^{g} \psi\right\rangle_{g H g^{-1}}=\frac{1}{\left|g H g^{-1}\right|} \sum_{x \in g H g^{-1}}{ }^{g} \varphi(x)^{\bar{g} \psi(x)} \\
&=\frac{1}{|H|} \sum_{x \in g H g^{-1}} \varphi\left(g^{-1} x g\right) \overline{\psi\left(g^{-1} x g\right)} \\
& y:=\stackrel{g^{-1} x g}{=} \frac{1}{|H|} \sum_{y \in H} \varphi(y) \overline{\psi(y)}=\langle\varphi, \psi\rangle_{H} .
\end{aligned}
$$


(b) As $N \unlhd G, g N g^{-1}=N$. Thus, if $\psi \in \operatorname{Irr}(N)$, then on the one hand ${ }^{g} \psi$ is also a character of $N$ by Exercise 20.2(d), and on the other hand it follows from (a) that $\left\langle{ }^{g} \psi,{ }^{g} \psi\right\rangle_{N}=\langle\psi, \psi\rangle_{N}=1$. Hence ${ }^{g} \psi$ is an irreducible character of $N$. Therefore, if ${ }^{g} \psi \in \operatorname{Irr}(N)$, then $\psi=g^{-1}\left({ }^{g} \psi\right) \in \operatorname{Irr}(N)$, as required.

(c) If $n \in N$ then so does $g^{-1} n g \forall g \in G$, hence

$$
\psi \uparrow_{N}^{G_{N}^{G}} \mathcal{G}_{N}(n)=\psi \uparrow_{N}^{G}(n)=\frac{1}{|N|} \sum_{g \in G} \psi\left(g^{-1} n g\right)=\frac{1}{|N|} \sum_{g \in G}{ }^{g} \psi(n)=\frac{\left|\mathcal{I}_{G}(\psi)\right|}{|N|} \sum_{g \in\left[G / \mathcal{I}_{G}(\psi)\right]}{ }^{g} \psi(n) .
$$

\section{Notation 20.4}

Given $N \unlhd G$ and $\psi \in \operatorname{Irr}(N)$, we set $\operatorname{Irr}(G \mid \psi):=\left\{\chi \in \operatorname{Irr}(G) \mid\left\langle\chi \downarrow_{N}^{G}, \psi\right\rangle_{N} \neq 0\right\}$.

\section{Theorem 20.5 (Clifford Theory)}

Let $N \unlhd G$. Let $\chi \in \operatorname{Irr}(G), \psi \in \operatorname{Irr}(N)$ and set $\mathcal{I}:=\mathcal{I}_{G}(\psi)$. Then the following assertions hold.

(a) If $\psi$ is a constituent of $\chi \downarrow_{N}^{G}$, then

$$
\chi \downarrow_{N}^{G}=e \sum_{g \in\left[G / \mathcal{I}_{G}(\psi)\right]}{ }^{g} \psi
$$

where $e=\left\langle\chi \downarrow_{N}^{G}, \psi\right\rangle_{N}=\left\langle\chi, \psi \uparrow_{N}^{G}\right\rangle_{G} \in \mathbb{Z}_{>0}$ is called the ramification index of $\chi$ in $N$ (or of $\psi$ in $G$ ). In particular, all the constituents of $\chi \downarrow_{N}^{G}$ have the same degree.

(b) Induction from $\mathcal{I}=\mathcal{I}_{G}(\psi)$ to $G$ induces a bijection

$$
\begin{aligned}
& \operatorname{lnd}_{\mathcal{I}}^{G}: \operatorname{Irr}(\mathcal{I} \mid \psi) \longrightarrow \operatorname{Irr}(G \mid \psi) \\
& \eta \quad \mapsto \quad \eta \uparrow_{\mathcal{I}}^{G}
\end{aligned}
$$

preserving ramification indices, i.e. $\left\langle\eta \downarrow_{N}^{\mathcal{I}}, \psi\right\rangle_{N}=\left\langle\eta \uparrow_{\mathcal{I}}^{G_{\mathcal{I}}} \downarrow_{N}^{\mathcal{I}}, \psi\right\rangle_{N}=$ e.

\section{Proof:}

(a) By Frobenius reciprocity, $\left\langle\chi, \psi \uparrow_{N}^{G}\right\rangle_{G}=\left\langle\chi \downarrow_{N}^{G}, \psi\right\rangle_{N} \neq 0$. Thus $x$ is a constituent of $\psi \uparrow_{N}^{G}$ and therefore $\chi \downarrow_{N}^{G}$ is a constituent of $\psi \uparrow_{N}^{G} \downarrow_{N}^{G}$.

Now, if $\eta \in \operatorname{Irr}(N)$ is an arbitrary constituent of $\chi \downarrow_{N}^{G}$ (i.e. $\left\langle\chi \downarrow_{N}^{G}, \eta\right\rangle_{N} \neq 0$ ) then by the above, we have

$$
\left\langle\psi \uparrow_{N}^{G} \downarrow_{N}^{G}, \eta\right\rangle_{N} \geqslant\left\langle\chi \downarrow_{N}^{G}, \eta\right\rangle_{N}>0 .
$$

Moroever, by Lemma 20.3(c) the constituents of $\psi \uparrow_{N}^{G} \downarrow_{N}^{G}$ are preciely $\left\{{ }^{g} \psi \mid g \in\left[G / \mathcal{I}_{G}(\psi)\right]\right\}$. Hence $\eta$ is $G$-conjugate to $\psi$. Furthermore, for every $g \in G$ we have

$$
\begin{aligned}
\left\langle\chi \downarrow_{N}^{G},{ }^{g} \psi\right\rangle_{N}=\frac{1}{|N|} \sum_{h \in N} \chi(h)^{g} \psi\left(h^{-1}\right) & =\frac{1}{|N|} \sum_{h \in N} \chi(h) \psi\left(g^{-1} h^{-1} g\right) \\
& \stackrel{x \in \mathcal{C l}(G)}{=} \frac{1}{|N|} \sum_{h \in N} \chi\left(g^{-1} h g\right) \psi\left(g^{-1} h^{-1} g\right) \\
s:=g^{-1} h g \in N & \frac{1}{|N|} \sum_{s \in N} \chi(s) \psi\left(s^{-1}\right)=\left\langle\chi \downarrow_{N}^{G}, \psi\right\rangle_{N}=e .
\end{aligned}
$$

Therefore, every $G$-conjugate ${ }^{g} \psi\left(g \in\left[G / \mathcal{I}_{G}(\psi)\right]\right)$ of $\psi$ occurs as a constituent of $\chi \downarrow_{N}^{G}$ with the same multiplicity e. The claim about the degrees is then clear as ${ }^{g} \psi(1)=\psi(1) \forall g \in G$. 
(b) Claim 1: $\eta \in \operatorname{Irr}(\mathcal{I} \mid \psi) \Rightarrow \eta \uparrow_{\mathcal{I}}^{G} \in \operatorname{Irr}(G \mid \psi)$.

Since $\mathcal{I}=\mathcal{I}_{\mathcal{I}}(\psi)$, (a) implies that $\eta \downarrow_{N}^{\mathcal{I}}=e^{\prime} \psi$ with $e^{\prime}=\left\langle\eta \downarrow_{N}^{\mathcal{I}}, \psi\right\rangle_{N}=\frac{\eta(1)}{\psi(1)}>0$. Now, let $\chi \in \operatorname{Irr}(G)$ be a constituent of $\eta \uparrow_{\mathcal{I}}^{G}$. By Frobenius Reciprocity we have

$$
0 \neq\left\langle\chi, \eta \uparrow_{\mathcal{I}}^{G}\right\rangle_{G}=\left\langle\chi \downarrow_{\mathcal{I}}^{G}, \eta\right\rangle_{\mathcal{I}}
$$

It follows that $\eta \downarrow_{N}^{\mathcal{I}}$ is a constituent of $\chi \downarrow_{\mathcal{I}}^{G_{\mathcal{L}}} \downarrow_{N}^{\mathcal{I}}$ and

$$
e:=\left\langle\chi \downarrow_{N}^{G}, \psi\right\rangle_{N}=\left\langle\chi \downarrow_{\mathcal{I}}^{G} \downarrow_{N}^{\mathcal{I}}, \psi\right\rangle_{N} \geqslant\left\langle\eta \downarrow_{N}^{\mathcal{I}}, \psi\right\rangle_{N}=e^{\prime}>0,
$$

hence $\chi \in \operatorname{Irr}(G \mid \psi)$. Moreover, by (a) we have $e=\left\langle\chi \downarrow_{N}^{G}{ }^{g} \psi\right\rangle_{N} \geqslant e^{\prime}$ for each $g \in G$. Therefore,

$$
\chi(1)=e \sum_{g \in[G / \mathcal{I}]}{ }^{g} \psi(1) \stackrel{(a)}{=} e|G: \mathcal{I}| \psi(1) \geqslant e^{\prime}|G: \mathcal{I}| \psi(1)=|G: \mathcal{I}| \eta(1)=\eta \uparrow_{\mathcal{I}}^{G}(1) \geqslant \chi(1) .
$$

Thus $e=e^{\prime}, \eta \uparrow_{\mathcal{I}}^{G}=\chi \in \operatorname{Irr}(G)$, and therefore $\eta \uparrow_{\mathcal{I}}^{G} \in \operatorname{Irr}(G \mid \psi)$.

Claim 2: $\chi \in \operatorname{Irr}(G \mid \psi) \Rightarrow \exists ! \eta \in \operatorname{Irr}(\mathcal{I} \mid \psi)$ such that $\left\langle\chi \downarrow_{\mathcal{I}}^{G}, \eta\right\rangle_{\mathcal{I}} \neq 0$.

Again by (a), as $\chi \in \operatorname{Irr}(G \mid \psi)$, we have $\chi \downarrow_{N}^{G}=e \sum_{g \in[G / \mathcal{I}]}{ }^{g} \psi$, where $e=\left\langle\chi \downarrow_{N}^{G}, \psi\right\rangle_{N} \in \mathbb{Z}_{>0}$. Therefore, there exists $\eta \in \operatorname{Irr}(\mathcal{I})$ such that

$$
\left\langle\chi \downarrow_{\mathcal{I}}^{G}, \eta\right\rangle_{\mathcal{I}} \neq 0 \neq\left\langle\eta \downarrow_{N}^{\mathcal{I}}, \psi\right\rangle_{N}
$$

because $\chi \downarrow_{N}^{G}=\chi \downarrow_{\mathcal{I}}^{G_{\mathcal{I}}} \downarrow_{N}^{\mathcal{I}}$, so in particular $\eta \in \operatorname{Irr}(\mathcal{I} \mid \psi)$. Hence existence holds and it remains to see that uniqueness holds. Again by Frobenius reciprocity we have $0 \neq\left\langle\chi, \eta \uparrow_{\mathcal{I}}^{G}\right\rangle_{G}$. By Claim 1 this forces $\chi=\eta \uparrow_{\mathcal{I}}^{G}$ and $\eta \downarrow_{N}^{\mathcal{I}}=e \psi$, so $e$ is also the ramification index of $\psi$ in $\mathcal{I}$.

Now, write $\chi \downarrow_{\mathcal{I}}^{G}=\sum_{\lambda \in \operatorname{Irr}(\mathcal{I})} a_{\lambda} \lambda=\sum_{\lambda \neq \eta} a_{\lambda} \lambda+a_{\eta} \eta$ with $a_{\lambda} \geqslant 0$ for each $\lambda \in \operatorname{Irr}(\mathcal{I})$ and $a_{\eta}>0$. It follows that

$$
\left(a_{\eta}-1\right) \eta \downarrow_{N}^{\mathcal{I}}+\sum_{\lambda \neq \eta} a_{\lambda} \lambda \downarrow_{N}^{\mathcal{I}}=\underbrace{{ }^{G}}_{=e \sum_{g \in[G / \mathcal{I}}{ }^{x} \downarrow^{G}}-\underbrace{\eta \downarrow_{N}^{\mathcal{I}}}_{=e \psi}=e \sum_{g \in[G / \mathcal{I}] \backslash[1]}{ }^{g} \psi .
$$

Since $\psi$ does not occur in this sum, but occurs in $\eta \downarrow_{N}^{\mathcal{I}}$, the only possibility is $a_{\eta}=1$ and $\lambda \notin \operatorname{Irr}(\mathcal{I} \mid \psi)$ for $\lambda \neq \eta$. Thus $\eta$ is uniquely determined as the only constituent of $\chi \downarrow_{\mathcal{I}}^{G}$ in $\operatorname{Irr}(\mathcal{I} \mid \psi)$.

Finally, Claims 1 and 2 prove that $\operatorname{Ind} d_{\mathcal{I}}^{G}: \operatorname{Irr}(\mathcal{I} \mid \psi) \longrightarrow \operatorname{Irr}(G \mid \psi), \eta \mapsto \eta \uparrow_{\mathcal{I}}^{G}$ is well-defined and bijective, and the proof of Claim 2 shows that the ramification indices are preserved.

\section{Example 13 (Normal subgroups of index 2)}

Let $N<G$ be a subgroup of index $|G: N|=2(\Rightarrow N \triangleleft G)$ and let $\chi \in \operatorname{Irr}(G)$, then either

(1) $\chi \downarrow_{N}^{G} \in \operatorname{Irr}(N)$, or

(2) $\chi \downarrow_{N}^{G}=\psi+{ }^{g} \psi$ for a $\psi \in \operatorname{Irr}(N)$ and a $g \in G \backslash N$.

Indeed, let $\psi \in \operatorname{Irr}(N)$ be a constituent of $\chi \downarrow_{N}^{G}$. Since $|G: N|=2$, we have $\mathcal{I}_{G}(\psi) \in\{N, G\}$. Theorem 20.5 yields the following:

- If $\mathcal{I}_{G}(\psi)=N$ then $\operatorname{Irr}\left(\mathcal{I}_{G}(\psi) \mid \psi\right)=\{\psi\}$ and $\psi \uparrow_{N}^{G}=\chi$, so that $e=1$ and we get $\chi \downarrow_{N}^{G}=\psi+{ }^{g} \psi$ for any $g \in G \backslash N$.

- If $\mathcal{I}_{G}(\psi)=G$ then $G / \mathcal{I}_{G}(\psi)=\{1\}$, so that

$$
\chi \downarrow_{N}^{G}=e \psi \quad \text { with } e=\left\langle\chi \downarrow_{N}^{G}, \psi\right\rangle_{N}=\left\langle\chi, \psi \uparrow_{N}^{G}\right\rangle_{G} .
$$


Moroever, by Lemma 20.3(c),

$$
\psi \uparrow \uparrow_{N}^{G}{ }_{N}^{G}=\left|\mathcal{I}_{G}(\psi): N\right| \sum_{g \in G / \mathcal{I}_{G}(\psi)}{ }^{g} \psi=2 \psi
$$

Hence

$$
2 \psi(1)=\psi \uparrow \uparrow_{N}^{G} \downarrow_{N}^{G}(1) \geqslant \chi \downarrow_{N}^{G}(1)=\chi(1)=e \psi(1) \quad \Rightarrow \quad e \leqslant 2 .
$$

Were $e=2$ then we would have $2 \psi(1)=\psi \uparrow \uparrow_{N}^{G}(1)$, hence $\chi=\psi \uparrow \uparrow_{N}^{G}$ and thus

$$
1=\left\langle\chi, \psi \uparrow_{N}^{G}\right\rangle_{G}=\left\langle\chi \downarrow_{N}^{G}, \psi\right\rangle_{N}=e=2
$$

a contradiction. Whence $e=1$, which implies that $\chi \downarrow_{N}^{G} \in \operatorname{Irr}(N)$. Moreover, $\psi \uparrow_{N}^{G}=\chi+\chi^{\prime}$ for some $\chi^{\prime} \in \operatorname{Irr}(G)$ such that $\chi^{\prime} \neq \chi$.

Remember that we have proved (Exercise 24, Sheet 6 ) that the degree of an irreducible character of a finite group $G$ divides the index of the centre $|G: Z(G)|$. The following consequence of Clifford's theorem due to $\mathrm{N}$. Itô provides us with yet a stronger divisibility criterion.

\section{Theorem 20.6 (Iтô)}

Let $A \leqslant G$ be an abelian subgroup of $G$ and let $\chi \in \operatorname{Irr}(G)$. Then the following assertions hold:

(a) $\chi(1) \leqslant|G: A|$; and

(b) if $A \unlhd G$, then $\chi(1)|| G: A \mid$.

\section{Proof:}

(a) Exercise 27, Sheet 7

(b) Let $\psi \in \operatorname{Irr}(A)$ be a constituent of $\chi \downarrow_{A}^{G}$, so that in other words $\chi \in \operatorname{Irr}(G \mid \psi)$. By Theorem 20.5(b) there exists $\eta \in \operatorname{Irr}\left(\mathcal{I}_{G}(\psi) \mid \psi\right)$ such that $\chi=\eta \uparrow_{\mathcal{I}_{G}^{G}(\psi)}^{G_{A}}$ and $\eta \downarrow_{A}^{\mathcal{I}_{A}(\psi)}=e \psi$ (proof of Claim 2). Now, as $A$ is abelian, all the irreducible characters of $A$ have degree 1 and for each $x \in A, \psi(x)$ is an $o(x)$-th root of unity. Hence $\forall x \in A$ we have

$$
|\eta(x)|=\left|\eta \downarrow_{A}^{\mathcal{I}_{G}(\psi)}(x)\right|=|e \psi(x)|=e|\psi(x)|=e \cdot 1=e=\eta(1) \quad \Rightarrow \quad A \subseteq Z(\eta) .
$$

Therefore, by Remark 17.5, we have

$$
\eta(1)|| \mathcal{I}_{G}(\psi): Z(\eta)||\left|\mathcal{I}_{G}(\psi): A\right|
$$

and since $\chi=\eta \uparrow_{\mathcal{I}_{G}(\psi)}^{G}$ it follows that

$$
\chi(1)=\left|G: \mathcal{I}_{G}(\psi)\right| \eta(1)|| G: \mathcal{I}_{G}(\psi)|\cdot| \mathcal{I}_{G}(\psi): A|=| G: A \mid .
$$

\section{The Theorem of Gallagher}

In the context of Clifford theory (Theorem 20.5) we understand that irreducibility of characters is preserved by induction from $\mathcal{I}_{G}(\psi)$ to $G$. Thus we need to understand induction of characters from $N$ to 
$\mathcal{I}_{G}(\psi)$, in particular what if $G=\mathcal{I}_{G}(\psi)$. What can be said about $\operatorname{Irr}(G \mid \psi)$ ?

\section{Lemma 21.1}

Let $N \unlhd G$ and let $\psi \in \operatorname{Irr}(N)$ such that $\mathcal{I}_{G}(\psi)=G$. Then

$$
\psi \uparrow \stackrel{G}{N}=\sum_{\chi \in \operatorname{lrr}(G)} e_{\chi} \chi
$$

where $e_{\chi}:=\left\langle\chi \downarrow{ }_{N}^{G}, \psi\right\rangle_{N}$ is the ramification index of $\chi$ in $N$; in particular

$$
\sum_{\chi \in \operatorname{lrr}(G)} e_{\chi}^{2}=|G: N|
$$

Proof: Write $\psi \uparrow_{N}^{G}=\sum_{\chi} \in \operatorname{Irr}(G) a_{\chi} \chi$ with suitable $a_{\chi}=\left\langle\chi, \psi \uparrow_{N}^{G}\right\rangle_{G}$. By Frobenius reciprocity, $a_{\chi} \neq 0$ if and only if $\chi \in \operatorname{Irr}(G \mid \psi)$. But by Theorem 20.5: if $\chi \in \operatorname{Irr}(G \mid \psi)$, then $\chi \downarrow_{N}^{G}=e_{\chi} \psi$, so that

$$
e_{\chi}=\left\langle\chi \downarrow_{N}^{G}, \psi\right\rangle_{N}=\left\langle\chi, \psi \uparrow_{N}^{G}\right\rangle_{G}=a_{\chi} .
$$

Therefore,

$$
|G: N| \psi(1)=\psi \uparrow_{N}^{G}(1)=\sum_{\chi \in \operatorname{Irr}(G)} a_{\chi} \chi(1)=\sum_{x \in \operatorname{Irr}(G)} e_{\chi} \chi(1)=\sum_{\chi \in \operatorname{lrr}(G)} e_{\chi}^{2} \psi(1)=\psi(1) \sum_{\chi \in \operatorname{lrr}(G)} e_{x}^{2}
$$

and it follows that $|G: N|=\sum_{X \in \operatorname{Ir}(G)} e_{\chi}^{2}$.

Therefore the multiplicities $\left\{e_{\chi}\right\}_{\chi \in \operatorname{Irr}(G)}$ behave like the irreducible character degrees of the factor group $G / N$. This is not a coincidence in many cases.

\section{Definition 21.2 (Extension of a character)}

Let $N \unlhd G$ and $\chi \in \operatorname{Irr}(G)$ such that $\psi:=\chi \downarrow_{N}^{G}$ is irreducible. Then we say that $\psi$ extends to $G$, and $\chi$ is an extension of $\psi$.

\section{Exercise 21.3 (Exercise 28, Sheet 7)}

Let $N \unlhd G$ and $\chi \in \operatorname{Irr}(G)$. Prove that

$$
\chi \downarrow_{N \uparrow}^{G} G_{N}^{G}=\operatorname{lnf}_{G / N}^{G}\left(\chi_{\text {reg }}\right) \cdot \chi
$$

where $\chi_{\text {reg }}$ is the regular character of $G / N$.

\section{Theorem 21.4 (GallaGHeR)}

Let $N \unlhd G$ and let $\chi \in \operatorname{Irr}(G)$ such that $\psi:=\chi \downarrow_{N}^{G} \in \operatorname{Irr}(N)$. Then

$$
\psi \uparrow \stackrel{G}{N}=\sum_{\lambda \in \operatorname{Irr}(G / N)} \lambda(1) \operatorname{lnf}_{G / N}^{G}(\lambda) \cdot \chi
$$

where the characters $\left\{\operatorname{Inf}_{G / N}^{G}(\lambda) \cdot \chi \mid \lambda \in \operatorname{Irr}(G / N)\right\}$ of $G$ are pairwise distinct and irreducible. 
Proof: By Exercise 21.3 we have $\psi \uparrow_{N}^{G}=\operatorname{Inf}_{G / N}^{G}\left(\chi_{\text {reg }}\right) \cdot \chi$, where $\chi_{\text {reg }}$ denotes the regular character of $G / N$. Recall that by Theorem 10.3, $\chi_{\text {reg }}=\sum_{\lambda \in \operatorname{Irr}(G / N)} \lambda(1) \lambda$, so that we have

$$
\psi \uparrow_{N}^{G}=\sum_{\lambda \in \operatorname{Irr}(G / N)} \lambda(1) \operatorname{lnf}_{G / N}^{G}(\lambda) \cdot \chi .
$$

Now, by Lemma 21.1, we have

$$
\begin{aligned}
|G: N|=\sum_{\chi \in \operatorname{lr}(G)} e_{\chi}^{2}=\left\langle\psi \uparrow_{N}^{G}, \psi \uparrow_{N}^{G}\right\rangle_{G} & =\sum_{\lambda, \mu \in \operatorname{lrr}(G / N)} \lambda(1) \mu(1)\left\langle\operatorname{Inf}_{G / N}^{G}(\lambda) \cdot \chi, \operatorname{Inf}_{G / N}^{G}(\mu) \cdot \chi\right\rangle_{G} \\
& \geqslant \sum_{\lambda \in \operatorname{Irr}(G / N)} \lambda(1)^{2}=|G: N| .
\end{aligned}
$$

Hence equality holds throughout. This proves that

$$
\left\langle\operatorname{lnf}_{G / N}^{G}(\lambda) \cdot \chi, \operatorname{lnf}_{G / N}^{G}(\mu) \cdot \chi\right\rangle=\delta_{\lambda \mu} .
$$

By Erercise 13.4, $\operatorname{Inf}_{G / N}^{G}(\lambda) \cdot \chi$ are characters of $G$ and hence the characters $\left\{\operatorname{Inf}_{G / N}^{G}(\lambda) \cdot \chi \mid \lambda \in \operatorname{Irr}(G / N)\right\}$ are irreducible and pairwise distinct, as claimed.

Therefore, given $\psi \in \operatorname{Irr}(N)$ which extends to $\chi \in \operatorname{Irr}(G)$, we get $\operatorname{Inf}_{G / N}^{G}(\lambda) \cdot \chi(\lambda \in \operatorname{Irr}(G / N))$ as further irreducible characters.

\section{Example 14}

Let $N<G$ with $|G: N|=2(\Rightarrow N \unlhd G)$ and let $\psi \in \operatorname{Irr}(N)$. We saw:

- if $\mathcal{I}_{G}(\psi)=N$ then $\psi \uparrow_{N}^{G} \in \operatorname{Irr}(G)$;

. if $\mathcal{I}_{G}(\psi)=G$ then $\psi$ extends to some $\chi \in \operatorname{Irr}(G)$ and $\psi^{G}=\chi+\chi^{\prime}$ with $\chi^{\prime} \in \operatorname{Irr}(G) \backslash\{\chi\}$. It follows that $\chi^{\prime}=\chi \cdot$ sign, where sign is the inflation of the sign character of $G / N \cong S_{2}$ to $G$. 
This appendix provides a short recap / introduction to some of the basic notions of module theory used in this lecture. Tensor products of vector spaces and algebraic integers are also recapped.

\section{Reference:}

[Rot10] J. J. Rotman. Advanced modern algebra. 2nd ed. Providence, RI: American Mathematical Society (AMS), 2010.

\section{A Modules}

Notation: Throughout this section we let $R=(R,+, \cdot)$ denote a unital associative ring.

\section{Definition A.1 (Left R-module)}

A left $R$-module is an ordered triple $(M,+, \cdot)$, where $M$ is a set endowed with an internal composition law

$$
\begin{aligned}
+: & M \times M \\
\left(m_{1}, m_{2}\right) & \mapsto M \\
& \mapsto m_{1}+m_{2}
\end{aligned}
$$

and an external composition law (or scalar multiplication)

$$
\begin{aligned}
\therefore \quad R \times M & \longrightarrow M \\
(r, m) & \mapsto r \cdot m
\end{aligned}
$$

satisfying the following axioms:

(M1) $(M,+)$ is an abelian group;

(M2) $\left(r_{1}+r_{2}\right) \cdot m=r_{1} \cdot m+r_{2} \cdot m$ for every $r_{1}, r_{2} \in R$ and every $m \in M$;

(M3) $r \cdot\left(m_{1}+m_{2}\right)=r \cdot m_{1}+r \cdot m_{2}$ for every $r \in R$ and every $m_{1}, m_{2} \in M$;

(M4) $(r s) \cdot m=r \cdot(s \cdot m)$ for every $r, s \in R$ and every $m \in M$.

(M5) $1_{R} \cdot m=m$ for every $m \in M$. 


\section{Remark A.2}

(a) Note that in this definition both the addition in the ring $R$ and in the module $M$ are denoted with the same symbol. Similarly both the internal multiplication in the ring $R$ and the external multiplication in the module $M$ are denoted with the same symbol. This is standard practice and should not lead to confusion.

(b) Right $R$-modules can be defined analogously using a right external composition law $\cdot: M \times R \longrightarrow R,(m, r) \mapsto m \cdot r$.

(c) Unless otherwise stated, in this lecture we always work with left modules. Hence we simply write " $R$-module" to mean "left $R$-module", and as usual with algebraic structures, we simply denote $R$-modules by their underlying sets.

(d) We often write $r m$ instead of $r \cdot m$.

\section{Example A.3}

(a) Modules over rings satisfy the same axioms as vector spaces over fields. Hence: vector spaces over a field $K$ are $K$-modules, and conversely.

(b) Abelian groups are $\mathbb{Z}$-modules, and conversely. (Check it! What is the external composition law?)

(b) If the ring $R$ is commutative, then any right module can be made into a left module by setting $r \cdot m:=m \cdot r \forall r \in R, \forall m \in M$, and conversely.

(Check it! Where does the commutativity come into play?)

\section{Definition A.4 (R-submodule)}

An $R$-submodule of an $R$-module $M$ is a subgroup $U \leqslant M$ such that $r \cdot u \in U \forall r \in R, \forall u \in U$.

\section{Properties A.5 (Direct sum of $R$-submodules)}

If $U_{1}, U_{2}$ are $R$-submodules of an $R$-module $M$, then so is $U_{1}+U_{2}:=\left\{u_{1}+u_{2} \mid u_{1} \in U_{1}, u_{2} \in U_{2}\right\}$. Such a sum $U_{1}+U_{2}$ is called a direct sum if $U_{1} \cap U_{2}=\{0\}$ and in this case we write $U_{1} \oplus U_{2}$.

\section{Definition A.6 (Morphisms)}

A (homo)morphism of $R$-modules (or an $R$-linear map, or an $R$-homomorphism) is a map of $R$ modules $\varphi: M \longrightarrow N$ such that:

(i) $\varphi\left(m_{1}+m_{2}\right)=\varphi\left(m_{1}\right)+\varphi\left(m_{2}\right) \forall m_{1}, m_{2} \in M$; and

(ii) $\varphi(r \cdot m)=r \cdot \varphi(m) \forall r \in R, \forall m \in M$.

A bijective morphism of $R$-modules is called an isomorphism (or an $R$-isomorphism), and we write $M \cong N$ if there exists an $R$-isomorphism between $M$ and $N$.

A morphism from an $R$-module to itself is called an endomorphism and a bijective endomorphism is called an automorphism. 


\section{Properties A.7}

If $\varphi: M \longrightarrow N$ is a morphism of $R$-modules, then the kernel

$$
\operatorname{ker}(\varphi):=\left\{m \in M \mid \varphi(m)=0_{N}\right\}
$$

of $\varphi$ is an $R$-submodule of $M$ and the image

$$
\operatorname{Im}(\varphi):=\varphi(M)=\{\varphi(m) \mid m \in M\}
$$

of $\varphi$ is an $R$-submodule of $N$. If $M=N$ and $\varphi$ is invertible, then the inverse is the usual set-theoretic inverse map $\varphi^{-1}$ and is also an $R$-homomorphism.

\section{Notation A.8}

Given $R$-modules $M$ and $N$, we set $\operatorname{Hom}_{R}(M, N):=\{\varphi: M \longrightarrow N \mid \varphi$ is an $R$-homomorphism $\}$. This is an abelian group for the pointwise addition of maps:

$$
\begin{aligned}
+: \operatorname{Hom}_{R}(M, N) \times \operatorname{Hom}_{R}(M, N) & \longrightarrow \operatorname{Hom}_{R}(M, N) \\
(\varphi, \psi) & \mapsto \varphi+\psi: M \longrightarrow N, m \mapsto \varphi(m)+\psi(m) .
\end{aligned}
$$

In case $N=M$, we write $\operatorname{End}_{R}(M):=\operatorname{Hom}_{R}(M, M)$ for the set of endomorphisms of $M$. This is a ring for the pointwise addition of maps and the usual composition of maps.

\section{Lemma-Definition A.9 (Quotients of modules)}

Let $U$ be an $R$-submodule of an $R$-module $M$. The quotient group $M / U$ can be endowed with the structure of an $R$-module in a natural way via the external composition law

$$
\begin{aligned}
R \times M / U & \longrightarrow M / U \\
(r, m+U) & \longmapsto r \cdot m+U .
\end{aligned}
$$

The canonical map $\pi: M \longrightarrow M / U, m \mapsto m+U$ is $R$-linear and we call it the canonical (or natural) homomorphism.

Proof: Similar proof as for groups/rings/vector spaces/...

\section{Theorem A.10 (The universal property of the quotient and the isomorphism theorems)}

(a) Universal property of the quotient: Let $\varphi: M \longrightarrow N$ be a homomorphism of $R$-modules. If $U$ is an $R$-submodule of $M$ such that $U \subseteq \operatorname{ker}(\varphi)$, then there exists a unique $R$-module homomorphism $\bar{\varphi}: M / U \longrightarrow N$ such that $\bar{\varphi} \circ \pi=\varphi$, or in other words such that the following diagram commutes:

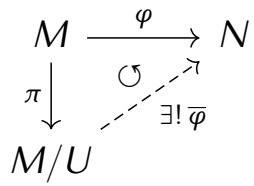

Concretely, $\bar{\varphi}(m+U)=\varphi(m) \forall m+U \in M / U$. 
(b) 1 st isomorphism theorem: With the notation of (a), if $U=\operatorname{ker}(\varphi)$, then

$$
\bar{\varphi}: M / \operatorname{ker}(\varphi) \longrightarrow \operatorname{Im}(\varphi)
$$

is an isomorphism of $R$-modules.

(c) 2nd isomorphism theorem: If $U_{1}, U_{2}$ are $R$-submodules of $M$, then so are $U_{1} \cap U_{2}$ and $U_{1}+U_{2}$, and there is an isomorphism of $R$-modules

$$
\left(U_{1}+U_{2}\right) / U_{2} \cong U_{1} /\left(U_{1} \cap U_{2}\right)
$$

(d) 3rd isomorphism theorem: If $U_{1} \subseteq U_{2}$ are $R$-submodules of $M$, then there is an isomorphism of $R$-modules

$$
\left(M / U_{1}\right) /\left(U_{2} / U_{1}\right) \cong M / U_{2}
$$

(e) Correspondence theorem: If $U$ is an $R$-submodule of $M$, then there is a bijection

$$
\begin{array}{cc}
\{R \text {-submodules } X \text { of } M \mid U \subseteq X\} & \longleftrightarrow\{R \text {-submodules of } M / U\} \\
X & \mapsto X / U \\
\pi^{-1}(Z) & \longleftrightarrow Z .
\end{array}
$$

Proof: Similar proof as for groups/rings/vector spaces/...

\section{Definition A.11 (Irreducible/reducible/completely reducible module)}

An $R$-module $M$ is called:

(a) simple (or irreducible) if it has exactly two submodules, namely the zero submodule 0 and itself;

(b) reducible if it admits a non-zero proper submodule $0 \subsetneq U \subsetneq M$;

(c) semisimple (or completely reducible) if it admits a direct sum decomposition into simple submodules.

Notice that the zero $R$-module 0 is neither reducible, nor irreducible, but it is completely reducible.

\section{B Algebras}

In this lecture we aim at studying modules over the group algebra, which are specific rings.

\section{Definition B.1 (Algebra)}

Let $R$ be a commutative ring.

(a) An $R$-algebra is an ordered quadruple $(A,+, \cdot, *)$ such that the following axioms hold:

(A1) $(A,+, \cdot)$ is a ring;

(A2) $(A,+, *)$ is a left $R$-module; and

(A3) $r *(a \cdot b)=(r * a) \cdot b=a \cdot(r * b) \forall a, b \in A, \forall r \in R$. 
(b) A map $f: A \rightarrow B$ between two $R$-algebras is called an algebra homomorphism iff:

(i) $f$ is a homomorphism of $R$-modules; and

(ii) $f$ is a ring homomorphism.

\section{Example 15}

(a) A commutative ring $R$ itself is an $R$-algebra.

[The internal composition law "." and the external composition law "*" coincide in this case.]

(b) For each $n \in \mathbb{Z} \geqslant 1$ the set $M_{n}(R)$ of $n \times n$-matrices with coefficients in a commutative ring $R$ is an $R$-algebra for its usual $R$-module and ring structures.

[Note: in particular $R$-algebras need not be commutative rings in general!]

(c) Let $K$ be a field. Then for each $n \in \mathbb{Z}_{\geqslant 1}$ the polynom ring $K\left[X_{1}, \ldots, X_{n}\right]$ is a $K$-algebra for its usual $K$-vector space and ring structure.

(d) If $K$ is a field and $V$ a finite-dimensional $K$-vector space, then $\operatorname{End}_{K}(V)$ is a $K$-algebra.

(e) $\mathbb{R}$ and $\mathbb{C}$ are $\mathbb{Q}$-algebras, $\mathbb{C}$ is an $\mathbb{R}$-algebra, ...

(f) Rings are $\mathbb{Z}$-algebras.

\section{Definition B.2 (Centre)}

The centre of an $R$-algebra $(A,+, \cdot *)$ is $Z(A):=\{a \in A \mid a \cdot b=b \cdot a \forall b \in A\}$.

\section{Tensor Products of Vector Spaces}

Throughout this section, we assume that $K$ is a field.

\section{Definition C.1 (Tensor product of vector spaces)}

Let $V, W$ be two finite-dimensional $K$-vector spaces with bases $B_{V}=\left\{v_{1}, \ldots, v_{n}\right\}$ and $B_{W}=$ $\left\{w_{1}, \ldots, w_{m}\right\}\left(m, n \in \mathbb{Z}_{\geqslant 0}\right)$ respectively. The tensor product of $V$ and $W$ (balanced) over $K$ is by definition the $(n \cdot m)$-dimensional $K$-vector space

$$
V \otimes_{K} W
$$

with basis $B_{V \otimes_{k} W}=\left\{v_{i} \otimes w_{j} \mid 1 \leqslant i \leqslant n, 1 \leqslant j \leqslant m\right\}$.

In this definition, you should understand the symbole " $v_{i} \otimes w_{j}$ " as an element that depends on both $v_{i}$ and $w_{j}$. The symbole " $\otimes$ " itself does not have any hidden meaning, it is simply a piece of notation: we may as well write something like $x\left(v_{i}, w_{j}\right)$ instead of " $v_{i} \otimes w_{j}$ ", but we have chosen to write " $v_{i} \otimes w_{j}$ ". 


\section{Properties C.2}

(a) An arbitrary element of $V \otimes_{K} W$ has the form

$$
\sum_{i=1}^{n} \sum_{j=1}^{m} \lambda_{i j}\left(v_{i} \otimes w_{j}\right) \quad \text { with }\left\{\lambda_{i j}\right\}_{\substack{1 \leqslant i \leqslant n \\ 1 \leqslant j \leqslant m}} \subseteq K .
$$

(b) The binary operation

$$
\begin{aligned}
B_{V} \times B_{W} & \longrightarrow B_{V \otimes_{K} W} \\
\left(v_{i}, w_{j}\right) & \mapsto v_{i} \otimes w_{j}
\end{aligned}
$$

can be extended by $\mathbb{C}$-linearity to

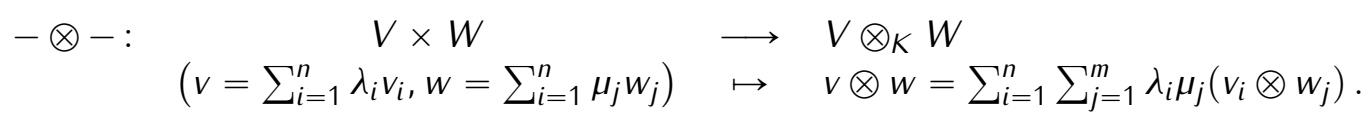

It follows that $\forall v \in V, w \in W, \lambda \in K$,

$$
v \otimes(\lambda w)=(\lambda v) \otimes w=\lambda(v \otimes w),
$$

and $\forall x_{1}, \ldots, x_{r} \in V, y_{1}, \ldots y_{s} \in W$,

$$
\left(\sum_{i=1}^{r} x_{i}\right) \otimes\left(\sum_{j=1}^{s} y_{j}\right)=\sum_{i=1}^{r} \sum_{j=1}^{s} x_{i} \otimes y_{j} .
$$

Thus any element of $V \otimes_{K} W$ may also be written as a $K$-linear combination of elements of the form $v \otimes w$ with $v \in V, w \in W$. In other words $\{v \otimes w \mid v \in V, w \in W\}$ generates $V \otimes_{K} W$ (although it is not a $K$-basis).

(c) Up to isomorphism $V \otimes_{K} W$ is independent of the choice of the $K$-bases of $V$ and $W$.

\section{Definition C.3 (Kronecker product)}

If $A=\left(A_{i j}\right)_{i j} \in M_{n}(K)$ and $B=\left(B_{r s}\right)_{r s} \in M_{m}(K)$ are two square matrices, then their Kronecker product (or tensor product) is the matrix

$$
A \otimes B=\left[\begin{array}{ccc}
A_{11} B & \ldots & A_{1 n} B \\
\vdots & & \vdots \\
A_{n 1} B & \ldots & A_{n n} B
\end{array}\right] \in M_{n \cdot m}(K)
$$

Notice that it is clear from the above definition that $\operatorname{Tr}(A \otimes B)=\operatorname{Tr}(A) \operatorname{Tr}(B)$. 


\section{Example 16}

E.g. the tensor product of two $2 \times 2$-matrices is of the form

$$
\left[\begin{array}{ll}
a & b \\
c & d
\end{array}\right] \otimes\left[\begin{array}{ll}
e & f \\
g & h
\end{array}\right]=\left[\begin{array}{cccc}
a e & a f & b e & b f \\
a g & a h & b g & b h \\
c e & c f & d e & d f \\
c g & c h & d g & d h
\end{array}\right] \in M_{4}(K) .
$$

\section{Lemma-Definition C.4 (Tensor product of K-endomorphisms)}

If $f_{1}: V \longrightarrow V$ and $f_{2}: W \longrightarrow W$ are two endomorphisms of finite-dimensional $K$-vector spaces $V$ and $W$, then the tensor product of $f_{1}$ and $f_{2}$ is the $K$-endomorphism $f_{1} \otimes f_{2}$ of $V \otimes_{K} W$ defined by

$$
\begin{aligned}
f_{1} \otimes f_{2}: \quad \begin{array}{l}
V \otimes_{K} W \\
v \otimes w
\end{array} & \longrightarrow \quad v \otimes_{K} W \\
v \otimes w & \left(f_{1} \otimes f_{2}\right)(v \otimes w):=f_{1}(v) \otimes f_{2}(w) .
\end{aligned}
$$

Furthermore, $\operatorname{Tr}\left(f_{1} \otimes f_{2}\right)=\operatorname{Tr}\left(f_{1}\right) \operatorname{Tr}\left(f_{2}\right)$.

Proof: It is straightforward to check that $f_{1} \otimes f_{2}$ is $K$-linear. Moreover, choosing ordered bases $B_{V}=$ $\left\{v_{1}, \ldots, v_{n}\right\}$ and $B_{W}=\left\{w_{1}, \ldots, w_{m}\right\}$ of $V$ and $W$ respectively, it is straightforward from the definitions to check that the matrix of $f_{1} \otimes f_{2}$ w.r.t. the ordered basis $B_{V \otimes_{k} W} w\left\{v_{i} \otimes w_{j} \mid 1 \leqslant i \leqslant n, 1 \leqslant j \leqslant m\right\}$ is the Kronecker product of the matrices of $f_{1}$ w.r.t. $B_{V}$ and of $f_{2}$ w.r.t. to $B_{W}$. The trace formula follows.

\section{Integrality and Algebraic Integers}

We recall/introduce here some notions of the Commutative Algebra lecture on integrality of ring elements. However, we are essentially interested in the field of complex numbers and its subring $\mathbb{Z}$.

\section{Definition D.1 (integral element, algebraic integer)}

Let $A$ be a subring of a commutative ring $B$.

(a) An element $b \in B$ is said to be integral over $A$ if $b$ is a root of monic polynomial $f \in A[X]$, that is $f(b)=0$ and $f$ is a polynomial of the form $X^{n}+a_{n-1} X^{n-1}+\ldots+a_{1} X+a_{0}$ with $a_{n-1}, \ldots, a_{0} \in A$. If all the elements of $B$ are integral over $A$, then we say that $B$ is integral over $A$.

(b) If $A=\mathbb{Z}$ and $B=\mathbb{C}$, an element $b \in \mathbb{C}$ integral over $\mathbb{Z}$ is called an algebraic integer.

\section{Theorem D.2}

Let $A \subseteq B$ be a subring of a commutative ring and let $b \in B$. TFAE:

(a) $b$ is integral over $A$;

(b) the ring $A[b]$ is finitely generated as an $A$-module;

(c) there exists a subring $S$ of $B$ containing $A$ and $b$ which is finitely generated as an $A$-module.

Recall that $A[b]$ denotes the subring of $B$ generated by $A$ and $b$. 


\section{Proof:}

(a) $\Rightarrow(\mathrm{b})$ : Let $a_{0}, \ldots, a_{n-1} \in A$ such that $b^{n}+a_{n-1} b^{n-1}+\ldots+a_{1} b+a_{0}=0$ (*). We prove that $A[b]$ is generated as an $A$-module by $1, b, \ldots, b^{n-1}$, i.e. $A[b]=A+A b+\ldots+A b^{n-1}$. Therefore it suffices to prove that $b^{k} \in A+A b+\ldots+A b^{n-1}=: C$ for every $k \geqslant n$. We proceed by induction on $k$ :

- If $k=n$, then $(*)$ yields $b^{n}=-a_{n-1} b^{n-1}-\ldots-a_{1} b-a_{0} \in C$.

- If $k>n$, then we may assume that $b^{n}, \ldots, b^{k-1} \in C$ by the induction hypothesis. Hence multiplying $(*)$ by $b^{k-n}$ yields

$$
b^{k}=-a_{n-1} b^{k-1}-\ldots-a_{1} b^{k-n+1}-a_{0} b^{k-n} \in C
$$

because $a_{n-1}, \ldots, a_{0}, b^{k-1}, \ldots, b^{k-n} \in C$.

(b) $\Rightarrow(\mathrm{c})$ : Set $S:=A[b]$.

(c) $\Rightarrow\left(\right.$ a): By assumption $A[b] \subseteq S=A x_{1}+\ldots+A x_{n}$, where $x_{1}, \ldots, x_{n} \in B, n \in \mathbb{Z}_{>0}$. Thus for each $1 \leqslant i \leqslant n$ we have $b x_{i}=\sum_{j=1}^{n} a_{i j} x_{j}$ for certain $a_{i j} \in A$. Set $x:=\left(x_{1}, \ldots, x_{n}\right)^{\operatorname{Tr}}$ and consider the $n \times n$-matrix $M:=b I_{n}-\left(a_{i j}\right)_{i j} \in M_{n}(S)$. Hence

$$
M x=0 \quad \Rightarrow \quad \operatorname{adj}(M) M x=0,
$$

where $\operatorname{adj}(M)$ is the adjugate matrix of $M$ (i.e. the transpose of its cofactor matrix). By the properties of the determinant (GDM), we have

$$
\operatorname{adj}(M) M=\operatorname{det}(M) I_{n},
$$

Hence $\operatorname{det}(M) x_{i}=0$ for each $1 \leqslant i \leqslant n$, and so $\operatorname{det}(M) s=0$ for every $s \in S$. As $1 \in S$ this gives us $\operatorname{det}(M)=0$. It now follows from the definition of $M$ that $b$ is a root of the monic polynomial $\operatorname{det}\left(X \cdot I_{n}-\left(a_{i j}\right)_{i j}\right) \in A[X]$, thus integral over $A$.

\section{Corollary D.3}

Let $A \subseteq B$ be a subring of a commutative ring. Then $\{b \in B \mid b$ integral over $A\}$ is a subring of $B$.

Proof: We need to prove that if $b, c \in B$ are integral over $A$, then so are $b+c$ and $b \cdot c$. By Theorem D.2(b) and its proof both $A[b]=A+A b+\ldots+A b^{n-1}$ and $A[c]=A+A c+\ldots+A c^{m-1}$ for some $n, m \in \mathbb{Z}_{>0}$. Thus $S:=A[b, c]$ is finitely generated as an $A$-module by $\left\{b^{i} c^{j} \mid 0 \leqslant i \leqslant n, 0 \leqslant j \leqslant m\right\}$. Theorem D.2(c) now yields that $b+c$ and $b \cdot c$ are integral over $A$ because they belong to $S$.

\section{Example 17}

All the elements of the ring $\mathbb{Z}[i]$ of Gaussian intergers are integral over $\mathbb{Z}$, hence algebraic integers, since $i$ is a root of $X^{2}+1 \in \mathbb{Z}[X]$.

\section{Lemma D.4}

If $b \in \mathbb{Q}$ is integral over $\mathbb{Z}$, then $b \in \mathbb{Z}$.

Proof: We may write $b=\frac{c}{d}$, where $c$ and $d$ are coprime integers and $d \geqslant 1$. By the hypothesis there exist $a_{0}, \ldots, a_{n-1} \in \mathbb{Z}$ such that

hence

$$
\frac{c^{n}}{d^{n}}+a_{n-1} \frac{c^{n-1}}{d^{n-1}}+\ldots+a_{1} \frac{c}{d}+a_{0}=0
$$

$$
c^{n}+\underbrace{d a_{n-1} c^{n-1}+\ldots+d^{n-1} a_{1}+d^{n} a_{0}}_{\text {divisible by } d}=0 .
$$

Thus $d \mid c^{n}$. As $\operatorname{gcd}(c, d)=1$ and $d \geqslant 1$ this is only possible if $d=1$, and we deduce that $b \in \mathbb{Z}$.

Clearly, the aforementionnend lemma can be generalised to integral domains (=Integritätsring) and their field of fractions. 


\section{General symbols}

$\mathbb{C}$

$\mathbb{F}_{q}$

$i$

$\operatorname{Id}_{M}$

$\operatorname{Im}(f)$

$\operatorname{ker}(\varphi)$

$\mathbb{N}$

$\mathbb{N}_{0}$

P

$\mathbb{Q}$

$\mathbb{R}$

$\mathbb{Z}$

$\mathbb{Z}_{\geqslant a}, \mathbb{Z}_{>a}, \mathbb{Z}_{\leqslant a}, \mathbb{Z}_{<a}$

$|X|$

$\delta_{i j}$

U

【

$\cap$

$\Gamma$

$\prod, \times$

$\oplus$

$\otimes$

$\varnothing$

$\forall$

$\exists$

$\cong$

$\bar{a}$

$a \mid b, a \nmid b$

$\left.f\right|_{S}$

\section{Group theory}

$A_{n}$

$C_{m}$

$C_{G}(x)$

$C(G)$

$D_{2 n}$

$\operatorname{Fix}_{x}(g)$ field of complex numbers

finite field with $q$ elements

$\sqrt{-1}$ in $\mathbb{C}$

identity map on the set $M$

image of the map $f$

kernel of the morphism $\varphi$

the natural numbers without 0

the natural numbers with 0

the prime numbers in $\mathbb{Z}$

field of rational numbers

field of real numbers

ring of integer numbers

$\{m \in \mathbb{Z} \mid m \geqslant a$ (resp. $m>a, m \geqslant a, m<a)\}$

cardinality of the set $X$

Kronecker's delta

union

disjoint union

intersection

summation symbol

cartesian product

direct sum

tensor product

empty set

for all

there exists

isomorphism

complex conjugate of $a \in \mathbb{C}$

$a$ divides $b, a$ does not divide $b$

restriction of the map $f$ to the subset $S$

alternating group on $n$ letters

cyclic group of order $m$ in multiplicative notation

centraliser of $x$ in $G$

set of conjugacy classes of $G$

dihedral group of order $2 n$

set of fixed points of $g$ on $X$ 


$$
\begin{aligned}
& {[G, G] \text { or } G^{\prime}} \\
& G / N \\
& G_{n}(K) \\
& H \leqslant G, H<G \\
& N \leqslant G \\
& N_{G}(H) \\
& \operatorname{PGL}_{n}(K) \\
& Q_{8} \\
& S_{n} \\
& \mathrm{SL}_{n}(K) \\
& \mathrm{S}_{p}(G) \\
& Z(G) \\
& \mathbb{Z} / m \mathbb{Z} \\
& |G| \\
& |G: H| \\
& {[x]} \\
& {[g, h]} \\
& \langle g\rangle \\
& \left\langle g \mid g^{m}=1\right\rangle
\end{aligned}
$$

\section{Rings and linear algebra}

$$
R[X]
$$

$$
R^{\times}
$$$$
\operatorname{char}(K)
$$$$
\text { det }
$$$$
\operatorname{dim}_{K}
$$$$
\operatorname{End}_{K}(V)
$$$$
\mathrm{GL}(V)
$$$$
\left\langle x_{1}, \cdots, x_{n}\right\rangle_{K}
$$$$
M_{n \times m}(K)
$$$$
M_{n}(K)
$$$$
\bar{K}
$$$$
\mathrm{Tr}
$$$$
W \leqslant V
$$$$
\left\{e_{1}, \cdots, e_{n}\right\}
$$$$
\left(e_{1}, \cdots, e_{n}\right)
$$

\section{Representations and characters}

$$
\begin{aligned}
& C_{1}, \ldots, C_{r} \\
& \widehat{C}_{1}, \ldots, \widehat{C_{r}} \\
& \mathcal{C} l(G) \\
& \mathcal{I}_{G}(\psi) \\
& \operatorname{Inf}_{G / N}^{G} \\
& \operatorname{Ind}_{H}^{C}, \uparrow_{H} \\
& \operatorname{Irr}(G)=\left\{\chi_{1}, \ldots, \chi_{r}\right\} \\
& \operatorname{Irr}(G \mid \psi)
\end{aligned}
$$

commutator subgroup of $G$ quotient group $G$ modulo $N$ general linear group over $K$ $H$ is a subgroup of $G$, resp. a proper subgroup $N$ is a normal subgroup $G$ normaliser of $H$ in $G$ projective linear group over $K$ quaternion group of order 8 symmetric group on $n$ letters special linear group over $K$ set of Sylow $p$-subgroups of the group $G$ centre of the group $G$ cyclic group of order $m$ in additive notation order of the group $G$ index of $H$ in $G$ conjugacy class of $x$ commutator of $g$ and $h$ cyclic group generated by $g$ cyclic group of order $m$ generated by $g$

ring of polynomials in an indeterminate $X$ over the ring $R$

group of units of the ring $R$ characteristic of the field $K$

determinant of a matrix/linear transformation $K$-dimension endomorphism ring of the $K$-vector space $V$ set of invertible linear transformations of the vector space $V$

$K$-linear span of the set $\left\{x_{1}, \cdots, x_{n}\right\}$ ring of $n \times m$-matrices with coefficients in $K$ ring of $n \times n$-matrices with coefficients in $K$ algebraic closure of the field $K$ trace of a matrix/linear transformation $W$ is a $K$-subspace of $V$ a basis of $K^{n}$ an ordered basis of $K^{n}$

the conjugacy classes of $G$ the class sums of $G$ $\mathbb{C}$-vector space of class functions on $G$ inertia group of $\psi$ in $G$ inflation from $G / N$ to $G$ induction from $H$ to $G$ set of irreducible characters of $G$ set of irreducible characters of $G$ above $\psi$ 


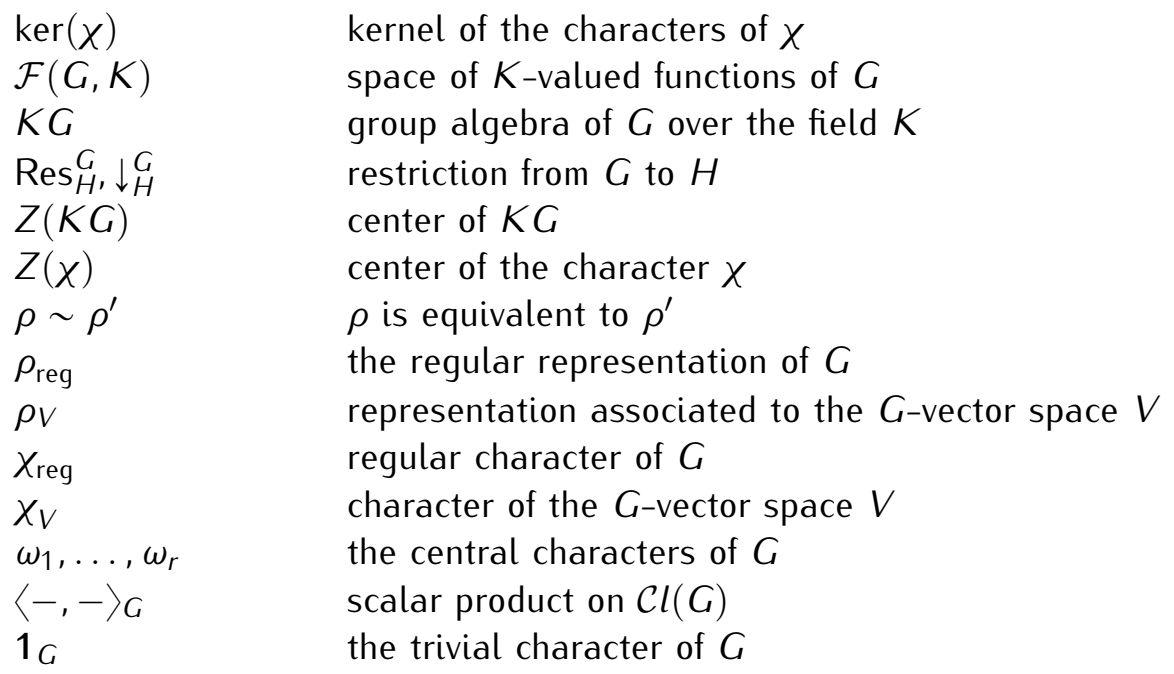

\section{Greek Alphabet}

\begin{tabular}{|c|c|c|}
\hline lower-case letter & upper-case letter & name \\
\hline$\alpha$ & $\bar{A}$ & alpha \\
\hline$\beta$ & B & beta \\
\hline$\gamma$ & $\Gamma$ & gamma \\
\hline$\delta$ & $\Delta$ & delta \\
\hline$\varepsilon, \epsilon$ & $E$ & epsilon \\
\hline$\zeta$ & $Z$ & zeta \\
\hline$\eta$ & $\mathrm{H}$ & eta \\
\hline$\theta$ & $\Theta$ & theta \\
\hline$l$ & $\mathrm{I}$ & iota \\
\hline$k$ & K & kappa \\
\hline$\lambda$ & $\Lambda$ & lambda \\
\hline$\mu$ & $\mathrm{M}$ & $\mathrm{mu}$ \\
\hline$v$ & $\mathrm{~N}$ & $\mathrm{nu}$ \\
\hline$\xi$ & $\equiv$ & $x i$ \\
\hline$O$ & $\mathrm{O}$ & omicron \\
\hline$\pi, \omega$ & $\Pi$ & pi \\
\hline$\rho, \varrho$ & $\mathrm{P}$ & rho \\
\hline$\sigma, \varsigma$ & $\Sigma$ & sigma \\
\hline$\tau$ & $\mathrm{T}$ & tau \\
\hline$v$ & $Y$ & upsilon \\
\hline$\phi, \varphi$ & $\Phi$ & phi \\
\hline$x$ & $X$ & chi \\
\hline$\psi$ & $\Psi$ & psi \\
\hline$\omega$ & $\Omega$ & omega \\
\hline
\end{tabular}

